\title{
TRAINEE SECTON
}

\author{
Alexander Frolkis \\ Jennifer Beatty \\ Michael Peplowski, \\ Noreen Singh \\ Craig Beers \\ Evan M. Beck, \\ Morley Hollenberg \\ Bryan Yipp \\ Paul L. Beck
}

Cumming School of Medicine, University of Calgary

Clin Invest Med 2018; 41 (2): E55-E126.

\section{Proceedings from the 7 th Annual University of Calgary Leaders in Medicine Research Symposium}

\begin{abstract}
On October 30th, 2015, the Leaders in Medicine (LIM) program at the Cumming School of Medicine, University of Calgary hosted its 7th Annual Research Symposium. Dr. Breanne Everett, President and CEO of Orpyx Medical Technologies and holder both of medical and MBA degrees from the University of Calgary, presented a lecture entitled "Marrying Business and Medicine: Toe-ing a Fine Line". The LIM symposium also provides a forum for both LIM and non-LIM medical students to present their research work in oral and poster presentation formats. This year over 100 students submitted their work and six oral presentations and 99 posters were presented. The oral presentations were as follows:

Ryan Lewinson Prediction of wedged insole-induced changes to knee joint moments during walking

Lindsey Logan Robotic measures provide insight on sensorimotor and cognitive impairments following traumatic brain injury

Jackie Mann What medication information do community doctors want to receive in discharge summaries for safer transfers?

Ashley Jensen Increased mortality associated with resident handoff periods at ten veterans administration medical centers

Jason Bau Keratinocyte growth factor protects against $C$. difficile-induced cell injury and death

Michael Keough A novel drug class promotes regeneration of central nervous system myelin by overcoming inhibitory scar molecules in vitro and in vivo

For further details on the University of Calgary Leaders in Medicine Program see "A Prescription that Addresses the Decline of Basic Science Education in Medical School" (Clinical and Investigative Medicine. 2014;37(5):E29). The LIM Symposium has the following objectives: (1) to showcase the variety of projects undertaken by students in the LIM Program as well as University of Calgary medical students; (2) to encourage medical student participation in research and special projects; (3) to inform students and faculty about the diversity of opportunities available for research and special projects during medical school and beyond; and, (4) to enhance student and staff interactions, with the ultimate goal being to enhance translational medicine improve health.
\end{abstract}

\section{Acknowledgments}

The Leaders in Medicine Research Symposium Organizing Committee would like to thank the following people: Keynote Speaker: Dr. Breanne Everett; Oral and Poster Competition Judges: Drs. S. Coderre, R. Musto, D. Hamilton, A. Flynn, V. Lekhi, S. Jarvis, A. Narendran, K. Rioux, T. Anderson, C. Temple-Oberle, I. Walker, S. Raj, A. Metcalfe, F. Zemp, J. Deniset, F. Lopes, S. Ruzycki, L. Bello-Espinosa, A. Bulloc, T. Williamson, T. Sajobi, M. Russell, B. Hallgrimsson, R. DeVinney, D. Hart, C. Hirota, P.-Y. von der Weid, E. Kurz, P. Kubes, K. Umeshappa and S. Warner; LIM Program Advisor: Michelle Selman; LIM Symposium Chairs: J. Beatty, M. Peplowski and N. Singh; and LIM Symposium Organizing Committee: A. Frolkis, L. Liu, L. Logan, A. Michaud, Z. Damani, S. Ramesh, C. Thornton, A. Jensen, J. Roberts, K. Witiuk, H. Leduc-Pessah, B. Shaw, M. Faria-Crowder, C. Marsters, A. Rogers, V. Iablokov, V. Vong, W. Nagesh, C. Beers, K. Reich, A. Forsyth, C. Kinlin and M. Bouwsema 


\section{Oral Presentations}

\section{Prediction of wedged insole-induced changes to knee joint moments during walking}

Ryan T. Lewinson ${ }^{1,2,3}$

Darren J. Stefanyshyn ${ }^{2,3}$

${ }^{1}$ Cumming School of Medicine

${ }^{2}$ Faculty of Kinesiology

3 Schulich School of Engineering, University of Calgary, Calgary, $\mathrm{AB}$

Introduction: Wedged footwear insoles/orthotics are commonly used for managing symptoms of medial knee osteoarthritis, a degenerative joint disease. Specifically, these insoles are intended to reduce the knee adduction moment (KAM) during stance phase of gait-a variable shown to be related to knee osteoarthritis disease severity and pain. However, for $12-33 \%$ of individuals, these insoles actually increase the KAM, potentially causing worsening of the disease. Unfortunately, it is not currently known how to predict the biomechanical effect that these insoles may have on a given patient, making personalized approaches to insole prescription challenging and potentially risking the health of a large number of individuals each year. The purpose of this study was to identify a method using two-dimensional video data for predicting the expected change in knee adduction moments with wedged insoles.

Methods: Knee adduction moments during walking were determined for healthy individuals $(\mathrm{n}=15)$ and individuals with medial knee osteoarthritis $(n=19)$ while wearing their own shoe without an insole (control), with a $6 \mathrm{~mm}$ medial wedge (i.e. elevated on medial aspect), and with a $6 \mathrm{~mm}$ lateral wedge (i.e., elevated on lateral aspect). Then, participants completed trials with each footwear condition where the movement was only a single-step, as opposed to walking, and only change in mediolateral positions of the knee joint center, lower-leg center of mass, ankle joint center and foot center of mass were evaluated. These variables were expressed as changes relative to the control condition and then used as predictors in regression equations-one for lateral wedges and one for medial wedges $(\alpha=0.05)$. Here, changes in KAMs during walking with each wedge type were the dependent variables. Thus, the regressions assessed the ability for single-step two-dimensional motion data to explain the variance in actual walking KAMs. Simulations were then performed to output predicted KAM changes using the two-dimensional single-step data as inputs, and these predicted KAMs were assessed relative to actual KAMs by computing the Predicted R-squared value $\left(r^{2} p, \alpha=0.05\right)$.

Results: Change in mediolateral positions of the lower extremity during a single-step significantly predicted change in KAMs during walking for the lateral wedge in both the healthy $\left(R^{2}=0.72, p=0.008\right)$ and knee osteoarthritis $\left(R^{2}=0.52\right.$, $p=0.026)$ groups, and also for the medial wedge in both the healthy $\left(R^{2}=0.67, p=0.016\right)$ and knee osteoarthritis $\left(R^{2}=0.54\right.$, $p=0.020)$ groups. Simulation-generated predicted KAMs were significantly correlated with actual KAMs for both healthy and osteoarthritic individuals with lateral wedges (healthy $r^{2}=0.44, p=0.007$; osteoarthritis $r^{2} p=0.30, p=0.016$ ), but not for medial wedges (healthy $r^{2} p=0.21, p=0.084 ; r^{2}=$ $0.10, p=0.19$ ).

Conclusion: The method of using two-dimensional position data from a single-step movement to predict walking biomechanics was successful. These data are relatively simple to collect and analyze relative to typical three-dimensional gait analysis, offering the possibility for future incorporation into a wedge prediction system.

Robotic measures provide insight on sensorimotor and cognitive impairments following traumatic brain injury

L. Logan ${ }^{1}$, J. Kenzie ${ }^{2}$

LI.J.A. Semrau ${ }^{2}$

LII.C.T. Debert ${ }^{2,3}$

LIII.S.P. Dukelow ${ }^{1,2,3}$

${ }^{1}$ Faculty of Kinesiology

${ }^{2}$ Hotchkiss Brain Institute

${ }^{3}$ Department of Clinical Neurosciences, University of Calgary, Calgary, $\mathrm{AB}$

Background: Traumatic Brain Injury (TBI) is a prominent cause of disability worldwide, and often results in significant neurologic deficits. Clinicians typically use observer-based ordinal scales to assess neurologic impairment after TBI; however, these measures can be affected by ceiling effects, insufficient reliability and observer bias. Our goal was to investigate the ability of three robotic tasks (a unilateral reaching task, a bilateral motor task and a bilateral motor task requiring cognitive attention) to identify sensorimotor and cognitive impairment as a result of TBI. 
Methods: Twenty-five TBI survivors (mild, moderate and severe) completed three tasks in the KINARM exoskeleton robot. The device supports the arms against gravity, and subjects view visual feedback of their movements through a horizontally mounted display. The first task, Visually Guided Reaching (VGR), is a single-target reaching task that measures unilateral motor function. In the second task, Object Hit $(\mathrm{OH})$, subjects use virtual paddles centered at the index finger to hit away balls that fall from the top of the screen. In the third task, Object Hit and Avoid (OHA), two shapes are first identified as targets; different shapes fall from the top of the screen, and shapes not identified as targets must be avoided. The $\mathrm{OH}$ and OHA tasks become more difficult with time. We compared robotic performance of TBI subjects within each task to neurologically intact control subjects $(>150)$, matched for age, sex and handedness. Additionally, we compared performance on tests of cognitive ability (the Montreal Cognitive Assessment or MOCA) to performance on robotic parameters.

Results: Compared to controls, subjects with TBI performed poorly on all robotic tasks. TBI subjects with cognitive impairment (a score of less than 26 on the MOCA) performed worse than those without cognitive impairment (a score of 26 or greater on the MOCA) on robotic measures. In the VGR task, individuals with cognitive impairment had significantly longer movement times $(\mathrm{r}=0.49, \mathrm{p}=0.01)$. For $\mathrm{OH}$ and $\mathrm{OHA}$, cognitive impairment correlated with fewer target hits $(\mathrm{OH}$ : $\mathrm{r}=0.46, \mathrm{p}=0.02$, OHA: $\mathrm{r}=0.47, \mathrm{p}=0.02)$ as well as more distractor hits (OHA: $\mathrm{r}=0.50, \mathrm{p}=0.01$ ). Cognitively impaired subjects also made more errors earlier in the task, when the task was easier $(\mathrm{OH}: \mathrm{r}=0.46, \mathrm{p}=0.02$, OHA: $\mathrm{r}=0.60$, $\mathrm{p}=0.002$ ). Also, for $\mathrm{OH}$ and $\mathrm{OHA}$, a larger percentage of TBI subjects who were cognitively impaired failed across all parameters (OH: $41 \%$ cognitively impaired and $15 \%$ without impairment; OHA: $34 \%$ and $7 \%$, respectively).

Conclusion: The robot tasks evaluated in this study were able to objectively quantify impairments in TBI patients. These robotic tasks demonstrate the impact of cognitive impairment on sensorimotor performance. Additionally, bilateral motor tasks (such as $\mathrm{OH}$ and $\mathrm{OHA}$ ) can evaluate the impact of cognitive attention on bilateral motor control. Our results show that robotic tasks are a robust method of assessing the relationship between sensorimotor and cognitive impairment. They offer a promising alternative to clinical assessments as a tool in the rehabilitation of TBI patients.

\section{What medication information do community doctors want to receive in discharge summaries for safer transfers?}

Jackie Mann, BA

Echo Enns, MD, CCFP, FHM

Melissa Amodia, RN

Cumming School of Medicine, University of Calgary, Calgary, $\mathrm{AB}$

Background: Transition from hospital to home represents a vulnerable time for patient safety, particularly surrounding medication changes. As part of the handoff to primary care, medication changed in hospital is disseminated to patients' receiving family doctors in the discharge summary, a one-way communication varying by author. As the Calgary Health Region works to implement a Required Organizational Practice to improve safety, we bring community doctors' insight into the process. Until now, hospitalists have never asked their audience for feedback on the usefulness of the medication information they relay. Learners will review how family doctors interpret the clarity, organization and usefulness of medication information found in hospitalists' discharge summaries and the improvements they would like to see.

Methods: We invited community family doctors who commonly receive discharged patients from the Peter Lougheed Centre Hospital in Northeast Calgary to walk through the discharge summaries they had received within the prior three months. Six doctors participated and twenty-three discharge summaries were evaluated. Participants were asked to rate and comment on the clarity, organization and completeness of the medication information. We asked how they determine what home medications a patient should take from the discharge summary and what improvements could be made.

Results: All community doctors found if there were no home meds listed, the overall use of the discharge summary was reduced. More than half (41\%) of the time, some home medications were not represented. Only 55\% of the time were community doctors confident that they knew what changes to home medications had been made in the hospital. The reason why the dose or schedule for a home medication changed was clear $55 \%$ of the time and $83 \%$ of interviewed doctors wanted to see the reasoning behind any changes. Perceived medication errors totalled a whopping 54\% and 69\% of medication information evaluated was considered "organized" but there 
was no consensus how the medications should be organized. One doctor found that changes/additions were well communicated; hospital admission of her patients could be seen as an opportunity to access scores of resources including investigations and consults not available in the community.

Lessons learned: In addition to technological builds suggested, hospitalists were recommended to include a full list of home medications and the rationale behind medication changes to ensure clarity of what was known and what was intended.

\section{Increased mortality associated with resident handoff periods at ten Veterans Administration Medical}

\section{Centers}

Ashley Jensen ${ }^{1,2}$

Joshua L. Denson ${ }^{1,3}$

Binhuan Wang ${ }^{4}$

Harry Saag 1

Yixin Fang ${ }^{4}$

Laura Evans ${ }^{1}$

Scott Sherman ${ }^{2,4}$

${ }^{1}$ Department of Medicine, New York University School of Medicine, New York University, New York, NY

${ }^{2}$ VA New York Harbor Healthcare System, New York, NY

3 Department of Pulmonary Sciences and Critical Care, University of Colorado, Denver, CO

${ }^{4}$ Department of Population Health, New York University, New York, NY

Background: Miscommunication during patient handoffs is a well-established contributor to medical error and adverse events. Monthly handoff periods where groups of trainee clinicians (interns and residents) rotate simultaneously, as part of their post-graduate training, may represent a vulnerable period for patients. Few studies have examined patient outcomes or mortality during these periods.

Purpose: Our objective was to test if these monthly handoffs by trainees were associated with increased in-hospital and post-discharge mortality.

Methods: A retrospective cohort analysis was conducted of adult patients discharged from one of 10 university-affiliated Veterans Affairs (VA) Medical Centers between July 1, 2008 and June 30, 2014. Electronic medical record data was obtained from the VA's national Corporate Data Warehouse and dates for trainee rotations were obtained directly from the 10 internal medicine residency programs. Hospital discharges were categorized into two cohorts: a handoff group for all patients admitted prior to and discharged within 7 days of the monthly rotation (resident, intern or both); and a control group of all remaining discharges. Intern, resident and instances of full (both intern and resident) handoff were each considered separately in the analysis. Potential confounders we included were age, sex, ethnicity, length of hospital stay, calendar month and the Elixhauser Comorbidity Index. The primary outcome was the unadjusted and adjusted in-hospital mortality. Additionally we assessed the 30- and 90-day mortality rates for each group.

Results: A total of 230,701 discharges were included. There were no significant differences in age, ethnicity, gender, length of stay, or Elixhauser score between groups. Unadjusted in-hospital mortality was significantly greater for all handoff groups (intern handoff only 3.5\%, Odds Ratio (OR) 1.77 [95\% Confidence Interval (CI) 1.58-1.98], $\mathrm{p}<0.0001$; resident handoff only 3.3\%, OR 1.64 [CI 1.46-1.83], p<0.0001; full handoff (intern and resident) 4.0\%, OR 1.95 [CI 1.63-2.33], $\mathrm{p}<0.0001)$ than for the control group $(2.0 \%)$. Following adjustment, only intern handoff remained a significant risk for increased in-hospital mortality (OR 1.14 [CI 1.07-1.21], $\mathrm{p}<0.0001)$. All handoff groups had significantly greater adjusted 30-day post-discharge mortality risk than controls (intern only: OR 1.20 [CI 1.15-1.26], p<0.0001; resident only OR 1.15 [CI 1.08-1.19], $\mathrm{p}<0.0001$; full handoff OR 1.09 [CI 1.02-1.17], $\mathrm{p}<0.05)$. Similarly, adjusted 90 -day post-discharge mortality was significantly greater in all handoff groups relative to controls (intern only OR 1.17 [1.12-1.23], $\mathrm{p}<0.0001$; resident only OR 1.14 [CI 1.08-1.19], $\mathrm{p}<0.0001$; full handoff OR 1.09 [CI 1.02-1.17], $\mathrm{p}<0.05$ ).

Conclusions: Monthly handoff periods were significantly associated with increased in-hospital and post-discharge mortality at both 30 and 90 days. Intern handoff, in particular, was associated with increased in-hospital mortality. Further research is needed to confirm these findings in other settings, and to identify and test strategies to reduce patient risk associated with monthly clinical trainee rotations. 


\section{Keratinocyte Growth Factor protects against C. difficile-induced cell injury and death}

Jason T. Bau

Al-Hasan

Y. Li

PL Beck

Department of Medicine, University of Calgary, Calgary, AB

Introduction: Clostridium difficile infections are a major cause of morbidity, both in hospital and in community settings. While infection with $C$. difficile can afflict all populations, particularly susceptible include patients who are elderly and/or have protracted hospital admissions. Primary treatment of $C$. difficile includes the use of the antibiotic agents metronidazole and vancomycin, although failure rates with these therapeutics have been reported to be as high as $25 \%$. For these reasons, novel therapeutics targeting $C$. difficile infections are required.

Keratinocyte growth factor (KGF) is a 164-amino acid growth factor involved in the activation of multiple proliferation pathways, particularly in the gut. Intriguingly, previous studies have reported KGF to have a role in protecting colonic tissues from TNBS- and DSS-induced models of colitis. Furthermore, KGF has been commercially developed as a therapeutic for preventing radiation- and chemotherapy-induced mucositis. A protective role for KGF, in the context of $C$. difficile infection, has not yet been elucidated.

Methods: Using multiple biochemical approaches (both in vitro and in vivo), we examined the role of KGF in protecting against $C$. difficile toxin-mediated cell death.

Results: Our results demonstrate a protective role of KGF against $C$. difficile toxin-mediated cell death. Treatment of human colorectal epithelial cells with $C$. difficile TxnA and TxnB resulted in cell death as measured by an LDH release and caspase 3 cleavage assays. These were further corroborated by immunostaining for markers of apoptosis; including PARP, caspase 3,7 and 8. Pretreatment of cells with KGF protected from both TxnA and TxnB-induced cell death. Additionally, KGF prevented toxin-mediated disruption of barrier function as measured by ZO- 1 tight junction staining. Using C57B1/6 mice, KGF injections were able to protect against TxnA-and TxnB- induced colitis in vivo as measured histologically and by assessment of intestinal permeability. Furthermore, KGF appeared to play a role in recovery post toxin injury.
Preliminary data suggests that specific signalling pathways involving ERK and AKT are involved.

Conclusions: KGF protected tissues from $C$. difficile-induced cell damage and death both in vitro and in vivo, which adds to our understanding of innate protective mechanisms involved in protecting against, and recovery from, $C$. difficile-induced intestinal injury. Modulation of KGF activity/signalling may represent a novel approach to preventing and/or treating this devastating disease.

\section{A novel drug class promotes regeneration of central nervous system myelin by overcoming inhibitory scar molecules in vitro and in vivo}

Michael B. Keough

James A. Rogers ${ }^{1}$

Ping Zhang 2

Samuel K. Jensen ${ }^{1}$

Tieyu Chen ${ }^{2}$

Mitchel G. Hurlbert ${ }^{1}$

Khalil S. Rawji ${ }^{1}$

Jason R. Plemel ${ }^{1}$

Marcus Koch ${ }^{1}$

Chang-Chun Ling ${ }^{2}$

V. Wee Yong 1

${ }^{1}$ Hotchkiss Brain Institute and Department of Clinical Neurosciences

${ }^{2}$ Department of Chemistry, University of Calgary, Calgary, $\mathrm{AB}$

Introduction: The central nervous system has limited capacity for tissue regeneration following injury, and this contributes to the irreversible decline seen in many neurological diseases. Myelin is an insulating substance produced by oligodendrocytes that wraps axons and enables rapid impulse propagation. Demyelination impairs this conduction and leaves denuded axons susceptible to further injury. As opposed to neurons, myelin can be regenerated after injury through maturation of nearby oligodendrocyte progenitors; however, this process is markedly impeded by the presence of a glial scar, in which astrocytes secrete a dense matrix of inhibitory molecules. Here we developed a novel drug class to target the synthesis of one of these molecular inhibitors, the chondroitin sulfate proteoglycans, with a goal of enhancing oligodendrocyte progenitor maturation and myelin regeneration. 
Methods: Mouse brains were dissociated and cells were cultured in vitro to study the inhibitory interaction between astrocyte-derived proteoglycans and oligodendrocyte progenitor maturation, in the presence of a novel proteoglycan synthesis inhibitor created by collaborators in the Department of Chemistry. This inhibitor is a fluorinated analog of $N$-acetylglucosamine (fluorosamine). Demyelination was induced in vivo by surgical injection of a detergent in the spinal cords of mice, and indices of myelin regeneration were measured following systemic fluorosamine treatment.

Results: Astrocyte-derived proteoglycans potently inhibited the maturation of oligodendrocyte progenitors in vitro; fluorosamine reduced the synthesis of proteoglycans, providing an environment more conducive for repair, as measured by improved oligodendrocyte progenitor maturation. Following experimental demyelination in vivo, fluorosamine reduced the burden of proteoglycans in lesions, resulting in accelerated maturation of oligodendrocyte progenitors, and most importantly, enhanced myelin regeneration.

Conclusions: We have developed a novel drug class to enhance central nervous system myelin regeneration by overcoming astrocyte-derived inhibitory scar molecules. Future work will assess fluorosamine in other animal models, such as traumatic spinal cord injury. Also, we will test a variety of newly synthesized fluorosamine derivatives to find molecules with even greater efficacy. The future of treatment for demyelinating disorders will greatly benefit from the development of myelin-regenerating therapies.

\section{Poster Presentations}

\section{Shared bacterial iron receptor antigens as potential multi-species vaccine components}

DM Curran $^{1}$

PJ Adamiak ${ }^{2}$

JE Fegan ${ }^{2}$

C Qian ${ }^{2}$

$\mathrm{RYu} \mathrm{u}^{2}$

AB Schryvers ${ }^{2}$

${ }^{1}$ Department of Ecosystems and Public Health, Faculty of Veterinary Medicine

2 Department of Microbiology, Immunology and Infectious Diseases, Cumming School of Medicine, University of Calgary, Calgary, AB

Background: Transferrins are glycoproteins that sequester and transport free iron within biological tissues. This process serves to recycle free iron, prevent iron toxicity and deprive potential pathogens of a necessary nutrient. To survive in this iron-scarce environment, many pathogenic bacteria utilize a receptor system to acquire iron from circulating transferrin. This system is present in a number of pathogens from the Pasteurellaceae family, notably Actinobacillus pleuropneumoniae, Actinobacillus suis, Haemophilus influenza, and Haemophilus parasuis, and the Neisseriaceae family, notably Neisseria meningitidis. The receptor system consists of two components, transferrin binding protein $\mathrm{A}(\mathrm{TbpA})$ and transferrin binding protein $\mathrm{B}(\mathrm{TbpB})$. TbpB, in particular, has shown promise as a novel vaccine antigen; however, $\mathrm{Tbp} B$ has also been shown to be hyper-variable as a result of frequent horizontal exchange transformation events occurring within the bacterial microenvironment. In this investigation we hypothesized that there is a common pool of $\mathrm{TbpB}$ variants that are exchanged between the significant disease-causing porcine pathogens: A. pleuropneumoniae; $A$. suis; and $\mathrm{H}$. parasuis.

Methods: This study investigated the genetic and immunogenic diversity of the transferrin receptor system from these three significant porcine pathogens. The dataset consisted of nucleic acid sequences obtained from a geographically- and temporally-diverse collection of isolates, consisting of $41 \mathrm{~A}$. pleuropneumoniae strains, $30 \mathrm{H}$. parasuis strains, and two $A$. suis strains. Genetic diversity was evaluated through phylogenetic and multiple sequence alignment analyses. To evaluate cross reactivity, pigs were immunized 
with the intact $\mathrm{TbpB}$ and two engineered subunits of $\mathrm{TbpB}$ : the TbpB N-lobe; and the TbpB C-lobe from the same $A$. pleuropneumoniae strain. The resulting sera were tested against a representative panel of $\mathrm{TbpBs}$

Results: Phylogenetic analyses demonstrated that the receptor protein sequences cluster independently of species, suggesting that there is genetic exchange of receptors between species. Immunogenic investigations further demonstrated a cross-reactive response, most notably with the highly conserved C-lobe sub-component.

Conclusions: These results suggest that there is a common reservoir for transferrin receptor antigenic variation amongst these pathogens, and provide support that a rationally designed TbpB-based vaccine may provide protection against all three pathogens, while accounting for antigenic variation.

\section{Fecal microbial transplants reduce antibiotic-resistant genes in patients with recurrent Clostridium difficile infection}

Braden Millan ${ }^{1}$

Heekuk Park ${ }^{1}$

Naomi Hotte ${ }^{1}$

Olivier Mathieu ${ }^{2}$

Pierre Burguiere ${ }^{2}$

Thomas A. Tompkins ${ }^{2}$

Dina $\mathrm{Kao}^{1}$

Karen L Madsen ${ }^{1}$

${ }^{1}$ Department of Medicine, University of Alberta, Edmonton, $\mathrm{AB}$

${ }^{2}$ Lallemand Health Solutions, Montreal, QC

Background: The gut harbors an enormous reservoir of antibiotic-resistance genes and potentially pathogenic organisms. Recurrent Clostridium difficile infection (RCDI) is associated with repeated antibiotic treatment and the enhanced growth of the antibiotic-resistant microbes.

Aims: This study analyzed the effect of fecal microbial transplantation (FMT) on the gut antibiotic-resistant profile in RCDI patients.

Methods: In a single center hospital-based study, patients with RCDI $(n=20)$ received FMT from universal donors via colonoscopy. Stool samples were collected from donors and patients prior to and following FMT. DNA was extracted and shotgun metagenomics performed using Illumina Nextera XT kit. Our assembled results as well as assembled libraries from the Human Microbiome Project (HMP) were aligned against the NCBI bacterial taxonomy database and the Comprehensive Antibiotic Resistance Database (CARD). Results were validated through a DNA microarray containing 354 antibiotic resistance genes.

Results: RCDI patients had significantly greater number and diversity of antibiotic resistance (ABR) genes compared with donors. Eleven patients were cured of RCDI following one FMT while ten required a second FMT. In all cases, a successful FMT was characterized by a decrease in ABR genes and this was maintained for up to a year. Taxonomic analysis showed the RCDI patients to be dominated by Proteobacteria with Escherichia coli and Klebsiella the most prevalent. Resolution of symptoms and decrease in ABR genes following FMT correlated with increased Bacteroidetes and Firmicutes and reduced Proteobacteria.

Conclusion: FMT was found to be effective in the eradication of pathogenic antibiotic-resistant organisms and eliminated antibiotic-resistance genes, suggesting a future role for FMT in treating patients who harbor multi-drug-resistant bacterial strains.

\section{Epidemiology, genomic and microbiome characterization of community-acquired Clostridium difficile infections}

Christina S. Thornton ${ }^{1}$

Alexander L. Greninger ${ }^{2}$

Charles Y. Chiu ${ }^{2}$

Dylan R. Pillai ${ }^{1}$

${ }^{1}$ Department of Microbiology, Immunology and Infectious Diseases, University of Calgary, Calgary, AB

2 Department of Laboratory Medicine, UCSF, San Francisco, CA, USA

Introduction: The most common cause of infectious diarrheal infection amongst hospitalized patients in North America is caused by Clostridium difficile infections (CDI). Clinically, nosocomial CDI has been well studied with several risk factors known for acquisition. Recently, several studies have described the onset of community-acquired CDI (CA-CDI); 20-45\% of all CDI cases have been attributed to community onset. CA-CDI patients are relatively free of traditional risk factors for nosocomial CDI. The hypervirulent strain (NAP1) has been associated with several CDI outbreaks worldwide. To better elucidate the impact of CA-CDI, the aim of this study 
was to identify CA-CDI cases in Calgary. $C$. difficile isolates from confirmed CA-CDI cases were characterized for antibiotic susceptibility testing, pulsed field gel electrophoresis and whole genome sequencing. Finally, microbiome analysis was done on CA-CDI samples to assess gut dysbiosis.

Methods: Patient data from 2012 to 2014 were included and 78 patients with $C$. difficile infection were contacted by telephone for questionnaire. Inclusion criteria included stool-positive-confirmed $C$. difficile infection. Whole genome sequencing was done by isolation of genomic DNA. Microbiome analysis was done by DNA isolation from stool and sequenced using Illumina MiSeq.

Results: Patients $(49 / 78 ; 62.8 \%)$ were contacted with the remainder considered lost to follow-up. Further characterization based on clinical timeline reclassified patients into the following categories: CA-CDI, greater than 12 weeks following discharge or no previous hospital admission (31/78; $39.7 \%)$, indeterminate, defined as $4-12$ weeks following discharge $(8 / 78 ; 10.3 \%)$, unknown, those patients who could not recall the status of their hospitalization prior to infection (10/78; 12.8\%); and lost to follow-up (29/78; 37.2\%). Overall, the majority of patients were female $(71.8 \%$ vs. $28.2 \%)$ and $50-59$ years of age (21.8\%). The most common co-morbidities included ulcerative colitis $(1 / 31 ; 3.2 \%)$, Crohn disease $(3 / 31$; $9.7 \%)$, celiac $(2 / 31 ; 6.5 \%)$ and irritable bowel syndrome $(7 / 31$; 22.6\%). Among the 31 patients with CA-CDI, 22 (71.0\%) had no recent hospitalizations within the last six months prior to infection. PFGE analysis was done on all $C$. difficile isolates collected and Tenover criteria were used to classify NAP types. Amongst the CA-CDI isolates, both NAP1 $(6 / 31 ; 19.4 \%)$ and NAP $4(6 / 31 ; 19.4 \%)$ were the most abundant and $7 / 31$ (22.6\%) did not identify with any NAP type. There was universal susceptibility amongst all isolates to metronidazole and only one isolate $(1 / 78 ; 1.3 \%)$ was resistant to vancomycin. Preliminary genomic sequencing shows several phylogenetic groupings consistent with PFGE. Additionally, preliminary microbiome analysis shows abundance of Bacteroidetes and Fusobactera phylum with variations in diversity between bona fide CA-CDI and those associated with hospitalization.

Conclusions: The impact of hypervirulent $C$. difficile strains in the community setting would affect our ability to control transmission outside the hospital setting and may serve as a persistent reservoir. Preliminary results have shown the not only the incidence of CA-CDI within Calgary, but the association with virulence and treatment failure. Future studies will look to characterize CA-CDI at a genomic level in comparison to nosocomial CDI. The impact of this on the health care system in costs, patient outcomes and burden has the ability to be immense given already limited resources.

\section{The proteolytic activity of Nepenthes pitcher fluid as a therapeutic for the treatment of celiac disease}

\author{
M Yang 1,2 \\ M Rey ${ }^{1}, \mathrm{E} \mathrm{Verdu}^{3}$ \\ DC Schriemer ${ }^{1}$
}

${ }^{1}$ Department of Biochemistry \& Molecular Biology, Faculty of Medicine, University of Calgary, Calgary, AB

${ }^{2}$ Leaders in Medicine Program, Faculty of Medicine, University of Calgary, Calgary, $\mathrm{AB}$

${ }^{3}$ Division of Gastroenterology, Department of Medicine, McMaster University, Hamilton, ON

Background: Celiac disease (CD) is a highly prevalent autoimmune disorder that is triggered by the incomplete digestion of immune stimulatory peptides in the gliadin fraction of dietary gluten. The basis for gliadin resistance to digestion is due to the abundance of $\mathrm{P}(\sim 15 \%)$ and $\mathrm{Q}(\sim 30 \%)$ residues in its protein sequence. Currently, no therapeutic product for $\mathrm{CD}$ exists but oral proteases aimed at efficiently digesting gliadin throughout the gastrointestinal tract have shown promise in advanced clinical trials. In this study, we propose that the proteolytic activity of Nepenthes plant extracts will be suited for an oral protease therapeutic forCD.

Methods: Digests of an immunodominant 33-mer peptide of $\alpha$-gliadin by the Nepenthes pitcher fluid was quantified by LC-MS. Digests and deamidation by tissue-transglutaminae-2 (TG-2) of gliadin extracts from wheat by the Nepenthes pitcher fluid proteins was performed in vitro in a dose dependent manner under simulated gastrointestinal conditions. Samples were analyzed by LC-MS/MS. In addition, all digests were performed with and compared with an equivalent dosage of pepsin.

Results: Quantification by LC-MS showed that Nepenthes pitcher fluid was able to digest the 33-mer peptide of $\alpha$-gliadin fully and efficiently even at a 10-times lower concentration than that of pepsin, which was only able to digest $\sim 7 \%$ of the peptide at a 1:10 molar ratio (enzyme: substrate). Quantitative and qualitative comparison of the LC-MS/MS data obtained from Nepenthes pitcher fluid digests of gliadin extracts appeared to show a net destruction of immunogenic peptides below initial levels and near minimal T-cell stimulatory levels as well as a significant net decrease in deamdiation with TG-2 in a dose-dependent manner, whereas it appeared that no net 
destruction or further release of immunogenic epitopes into solution was observed when compared with equivalent dosages of pepsin.

Conclusions: A novel protease formulation in Nepenthes pitcher fluid appeared to efficiently process immunogenic epitopes of gliadin under gastronintestinal conditions, supporting the potential of the proposed protease formulation as an effective oral therapeutic candidate for CD.

\section{Synergistic effects of concomitant human rhinovirus and bacterial exposures in induction of CCL20 from human bronchial epithelial cells}

Barbara Maciejewski

David Proud

Airway Inflammation Research Group, Snyder Institute for Chronic Diseases, Physiology and Pharmacology, University of Calgary, Calgary, $\mathrm{AB}$

Background: Human rhinovirus (HRV) infections trigger acute attacks of lower airway diseases, such as chronic obstructive pulmonary disease (COPD). These attacks are life-threatening and lead to loss of lung function. Airway epithelial cells are the primary site of HRV infection. Infected cells release both proinflammatory mediators and antiviral/host defense molecules that can regulate viral growth and reproduction. CCL20 stimulates both innate and adaptive immune responses by recruiting immature dendritic cells to the airway mucosa. The airways of patients with COPD are often chronically colonized by bacteria such as nontypeable Haemophilus influenzae (NTHI). Few studies have examined the effect of bacterial infection on epithelial response to HRV. We hypothesize that the combination of HRV and NTHI infection modulates CCL20 production relative to that observed with either stimulus alone.

Methods: Primary human bronchial epithelial cells were isolated from non-transplanted donor lungs and cultured in Bronchial Epithelial Growth Medium. Cells were grown to full confluence and were stimulated either with medium alone or with medium containing HRV, NTHI or a combination of HRV and NTHI. After six, 12 and 24 hours, supernatants were collected for protein assays (ELISA) and cells harvested for mRNA analysis
(RT-PCR). HRV was rendered replication-deficient by exposure to UV light for five minutes. Cells were exposed to TLR agonists to mimic bacterial responses. Cells were treated with MAPK inhibitors to isolate pathway elements involved in HRV/NTHI synergy. Actinomycin $\mathrm{D}$ (ActD) assays were used to determine if mRNA stabilization contributed to the observed responses.

Results: While both HRV and the bacteria alone induced production of CCL20, the combination of bacteria and HRV caused a synergistic expression of both protein and mRNA levels. Time-course data indicated that HRV induced maximum CCL20 steady-state mRNA production at six hours, which then dropped off at 12 and 24 hours, while protein levels increased gradually throughout the time-course. When HRV and NTHI were combined, CCL20 mRNA levels were maintained across all three time-points; however, ActD assays did not show mRNA stabilization in the presence of both NTHI and HRV. CCL20 protein induction was the same for normal and replication-deficient HRV. A selective MEK1/MEK2 inhibitor, U0126, appeared to abrogate viral-bacterial synergy.

Conclusion: HRV and bacteria synergistically induced CCL20 mRNA and protein expression. NTHI appeared to stabilize CCL20 mRNA induced by HRV in time-course experiments, but ActD assays did not show mRNA stabilization. CCL20 protein induction appeared to be mediated by HRV cellular receptor signalling. Synergy may be associated with MEK1/MEK2 signalling. This study provides important new insights into the immune response to HRV in airways already colonized by bacteria and may identify novel target for therapeutic interventions. 
Cardiorespiratory toxicity of environmentally relevant zinc oxide nanoparticles in the freshwater fish Catostomus commersonii

Robin Bessemer

Kathryn Butler

Louise Tunnah

Neal Callaghan

Amanda Rundle

Suzie Currie

Christopher Dieni

Tyson MacCormack

\section{Mount Allison University, Sackville, NB}

Introduction: The inhalation of zinc oxide engineered nanomaterials (ENMs) has been linked to cardiorespiratory dysfunction in mammalian models but the effects of aquatic ENM exposure on fish have not been fully investigated. Nano-zinc oxide ( $\mathrm{nZnO}$ ) is widely used in consumer products such as sunscreens and can make its way into aquatic ecosystems from domestic and commercial wastewater. This study examined the impact of an environmentally relevant $\mathrm{nZnO}$ formulation on cardiorespiratory function and energy metabolism in the white sucker (Catostomus commersonii), a freshwater teleost fish.

Methods: Thirty white suckers were caught by Fyke trap in Silver Lake (Sackville, NB, Canada). Independent control and $\mathrm{nZnO}$ treatment groups were run for ECG studies and intermittent closed-loop respirometry studies. A $1.0 \mathrm{mg} / \mathrm{mL}$ $\mathrm{nZnO}$ suspension was added to the tank of treatment fish to achieve a final nominal exposure dose of $1.0 \mathrm{mg} / \mathrm{L}$ ( $\mathrm{n}=6$ for ECG, $\mathrm{n}=8$ for respirometry. To investigate the impact of $\mathrm{nZnO}$ exposure on membrane proteins involved in osmoregulation, gill $\mathrm{Na}^{+} / \mathrm{K}^{+}$-ATPase activity was measured.

Osmotic homeostasis in the whole animal was assessed using plasma osmolality and chloride concentration. Plasma lactate and cortisol levels were assessed as indicators of physiological stress. Cellular stress in gill tissue was also studied by quantifying the expression of the stress-inducible heat shock proteins (HSP) HSP70 and HSP90 using Western blotting.

Results: Evidence of oxidative and cellular stress was present in gill tissue, including increases in malondialdehyde levels, heat shock protein (HSP) expression, and caspase 3/7 activity. Gill $\mathrm{Na}^{+} / \mathrm{K}^{+}$-ATPase activity was also higher by approximately three-fold in $\mathrm{nZnO}$-treated fish, likely in response to increased epithelial permeability or structural remodelling. Despite evidence of gill toxicity, plasma cortisol and lactate levels did not change in animals exposed to $1.0 \mathrm{mg} / \mathrm{L} \mathrm{nZnO}$. White suckers also exhibited a $35 \%$ decrease in heart rate during $\mathrm{nZnO}$ exposure, with no significant changes in resting oxygen consumption or tissue energy stores.

Conclusion: Our results suggest that tissue damage or cellular stress resulting from $\mathrm{nZnO}$ exposure activates gill neuroepithelial cells, triggering a whole-animal hypoxic response. An increase in parasympathetic nervous signalling will decrease heart rate and may reduce energy demand, even in the face of an environmental toxicant. We have shown that acute exposure to $\mathrm{nZnO}$ is toxic to white suckers and that ENMs have the potential to negatively impact cardiorespiratory function in adult fish.

\section{Intestinal epithelial barrier enhancement by serine proteases requires ongoing proteolysis, activation of epidermal growth factor receptor, and Src activity.}

\section{Kelcie A. Lahey \\ Wallace K. MacNaughton}

Inflammation Research Group, Department of Physiology and Pharmacology, Snyder Institute for Chronic Diseases, University of Calgary, Calgary, AB

Introduction: The primary determinant of epithelial barrier function and paracellular transport is the tight junction. Disrupted barrier function is associated with the development of intestinal diseases including the inflammatory bowel diseases, Crohn's disease and ulcerative colitis. Apical exposure of the intestinal epithelium to serine proteases such as trypsin or matriptase results in an increase in transepithelial electrical resistance (TER), a measure of enhanced barrier function. We aimed to determine the requirement for proteolytic activity and epidermal growth factor receptor (EGFR) activation in initiating and maintaining enhanced barrier function following protease treatment, as well as the mechanism by which activation occurs.

Methods: 1) TER across confluent SCBN epithelial cell monolayers was measured in Ussing chambers. Proteolytic activity was inhibited by 10,30 , or $60 \mu \mathrm{g} / \mathrm{ml}$ soybean trypsin inhibitor (SBTI) or $300 \mathrm{nM}$ aprotinin at 10 minutes (start of plateau phase of increased TER) or 30 minutes (after sustained phase had been established) after protease stimulation. 2) EGFR activity was blocked by the selective EGFR tyrosine kinase inhibitor, PD153035 $(1 \mu \mathrm{M})$ before or after trypsin 
stimulation. 3) Src has been shown to mediate intracellular EGFR transactivation in other cell models. SCBN monolayers were treated with the Src inhibitor, PP1 $(0.5,1,5,10$, and 20 $\mu \mathrm{M}$ ) before or after trypsin stimulation. 4) Since EGFR can be transactivated extracellularly by matrix metalloproteinase (MMP)-mediated cleavage of membrane-tethered ligands, SCBN monolayers were pre- treated with the MMP inhibitors, marimastat $(0.1,0.5,1,5$, and $10 \mu \mathrm{M})$ or $\operatorname{GM6001}(1,5,10$, 20 , and $30 \mu \mathrm{M})$, then stimulated with trypsin.

Results: 1) Trypsin caused a rapid and sustained increase in TER $\left(\max \Delta \mathrm{TER}=233.5 \pm 25 \Omega^{*} \mathrm{~cm}^{2}\right)$. SBTI at 30 and $60 \mu \mathrm{g} /$ $\mathrm{ml}$ decreased the trypsin-induced increase in TER by $56 \pm 4 \%$ and $67 \pm 5 \%$ at 10 minutes and by $61 \pm 4 \%$ and $74 \pm 4 \%$ at 30 minutes. $300 \mathrm{nM}$ aprotinin decreased the matriptase-induced increase in TER by $85 \pm 7 \%$ at 30 minutes. 2) Pre-treatment with $1 \mu \mathrm{M}$ PD153035 decreased the maximal and sustained responses to trypsin by $43 \pm 3 \%$ and $84 \pm 3 \%$, respectively. EGFR inhibition did not block or delay the initial onset of trypsin-induced increase in TER. Inhibiting EGFR 10 minutes after trypsin stimulation caused a $50 \pm 4 \%$ decrease in the trypsin-induced increased TER. 3) Pre-treatment with PP1 significantly decreased the peak trypsin response in a concentration-dependent manner. 4) Pre-treatment with marimastat and GM6001 had no significant effect on the protease-mediated increasedTER.

Conclusions: Our data show a requirement for ongoing proteolytic activity and EGFR activation to sustain protease-induced intestinal barrier enhancement. This enhancement is also dependent on the activity of Src, but not MMPs. By elucidating mechanisms by which epithelial barrier function is regulated, our work could contribute to the discovery of novel therapeutic targets for intestinal inflammatory disease, such as IBD, that are characterized by a disrupted epithelial barrier.

\section{RGS2 as a potential modulator of myometrial contractility in labour}

\author{
Hayley S. Levinson ${ }^{1}$ \\ Daniela Urrego ${ }^{1}$ \\ Stephen Wood ${ }^{2}$ \\ Robert Newton ${ }^{3}$ \\ Donna M. Slater ${ }^{1,2}$
}

${ }^{1}$ Physiology \& Pharmacology

${ }^{2}$ Obstetrics \& Gynecology

${ }^{3}$ Cell Biology \& Anatomy, University of Calgary, Calgary, AB

Introduction: In myometrial smooth muscle, activation of Gaq-coupled, G-protein coupled receptors (GPCRs) increase the level of intracellular calcium resulting in smooth muscle contraction. Compounds known to stimulate contractility at labour, such as oxytocin and prostaglandin $F 2 \alpha$, exert their effects by binding to G $\alpha$ q- coupled GPCRs. Understanding the mechanisms regulating uterine contractility is crucial to improving the clinical management of preterm labour. The regulator of G-protein signalling 2 (RGS2) can modulate smooth muscle contractility by terminating Gaq-coupled GPCR signalling. We hypothesize that RGS2 in the myometrium may contribute to the regulation of myometrial contraction during labour. Prostaglandin E2 (PGE2), a lipid mediator known to play a role in labour, enhances RGS2 expression in human myometrial cells, and in other smooth muscle types glucocorticoid further increased expression. Since glucocorticoids are used in the treatment of preterm labour and prostaglandins are known to modulate contractility at labour, we sought to

(i) determine the pattern of RGS2 expression in pregnant tissue at term and preterm labour; (ii) use a cell model to assess the regulation of RGS2 by PGE2 and dexamethasone. The aim of this research is to gain a better understanding of the process of human parturition, with the long-term goal of providing safer and more effective management of preterm labour.

Methods: Myometrial biopsies were collected from two regions of the uterus, upper segment (US) and lower segment (LS) at Caesarean deliveries. Groups were characterized as; term labour (TL), term non-labour (TNL), preterm labour (PTL), and preterm non-labour (PTNL). RNA was isolated from these biopsies and the RGS2 expression profile characterized. Myometrial smooth muscle cells were isolated and cultured and used as a model to assess RGS2 expression and regulation. An RGS2 mRNA expression time course ranging from 60 minutes to 18 hours post-treatment was conducted in 
cells treated with PGE2 in the presence or absence of dexamethasone. To investigate which PGE2 receptors regulate RGS2 expression, cells were treated with selective EP1-4 agonists alone, or PGE2 pretreated with selective EP2 or EP4 antagonists.

Results: The expression of RGS2 is increased in myometrium from the lower segment of the uterus in TL compared with TNL women. In contrast, the expression of RGS2 is decreased in myometrium from both the upper and lower segments of the uterus in PTL compared with PTNL. Myometrial cells, treated with PGE2 and dexamethasone, demonstrated higher expression of RGS2 mRNA at all time points compared with either treatment alone. Selective EP2 and EP4 agonists, but not selective EP1 or EP3 agonists, increased RGS2 expression compared with non-treated cells. Pre-treatment of cells with either an EP2 or an EP4 antagonist did not affect the PGE2 mediated increase in RGS2 gene expression.

Conclusion: RGS2 seems to be regulated in the myometrium with the onset of labour, both at term and preterm, supporting the hypothesis that RGS2 may be involved in modulating uterine contractility. We have demonstrated that RGS2 expression can be increased by co-treatment with PGE2 and dexamethasone, and that this is effect is likely mediated by EP2/EP4 activation.

\section{The effect of Cyclooxygenase-2 Inhibition on proximal tubular function of the kidney in humans}

Ann A. Zalucky

Bill Kangarloo

Darlene Y. Sola

Sofia B. Ahmed

University of Calgary, Calgary, $\mathrm{AB}$

Background: Non-steroidal anti-inflammatory drugs (NSAIDs) selective for cyclooxygenase-2 (COX-2) inhibition have been associated with cardio-renal toxicity, although the mechanism remains unclear. The renin- angiotensin-system (RAS) is a key player in blood pressure (BP) regulation and has been implicated as a possible contributing factor. The role for altered sodium handling at the proximal tubule as mediated by the RAS following COX-2 inhibition is unclear.

Objective: The objective of this study was to determine the change in sodium reabsorption at the proximal tubule in healthy humans in response to COX-2 inhibition.
Methods: Healthy subjects studied in high-salt balance were studied 14 days pre- and post-daily ingestion of $200 \mathrm{mg}$ celecoxib. The set-point of proximal renal tubular sodium excretion was estimated by the well-accepted surrogate fractional excretion of lithium, which is not altered by changes in diet. Serum and urine lithium was measured by graphite furnace atomic absorption spectroscopy. Fractional excretion of lithium was calculated using the following formula: (lithium urinary $\mathrm{x}$ creatinine serum/ lithium serum $\mathrm{x}$ creatinine urinary).

Primary outcome measure: The change in fractional extraction of lithium (FELi) after a two week intervention with celecoxib.

Results: Twelve healthy subjects ( $58 \%$ male, age $37 \pm 10$ years, SBP/DBP $118 \pm 13 / 84 \pm 11 \mathrm{mmHg}$, filtration fraction (FF), $14 \pm 2$ ) demonstrated a mean FELi of $6.7 \pm 3.4 \%$, which revealed no significant change post intervention (FELi $6.8 \pm 3.6 \%$; $\mathrm{p}=$ 0.4). Pre-COX-2 inhibition FELi showed a significant negative correlation with plasma renin activity (PRA) $(\mathrm{r}=-0.74 ; \mathrm{p}=0.006)$ and a positive correlation with $\mathrm{FF}(\mathrm{r}=0.57$; $\mathrm{p}=0.067)$. These associations remained post-celecoxib intervention (PRA: $\mathrm{p}=0.9, \mathrm{FF}: \mathrm{p}=0.4$ pre- vs post- celecoxib); however, these associations were not significant. No changes in $\mathrm{BP}$ or renal function were observed post intervention.

Conclusion: COX-2 inhibition in healthy humans does not result in a change in the set-point for renal sodium excretion. This may imply that COX-2 inhibitors have limited toxicity in the healthy non-chronic NSAID user. Further investigation on the role of RAS-mediated sodium handling at the proximal tubule with COX-2 inhibition is required. 


\section{Dual role for NLRP3 in Enterohemorrhagic Escherichia coli-induced inflammation and kidney injury}

Adom Bondzi-Simpson 1,3,4

Hyunjae Chung ${ }^{1,3}$

Arthur Lau ${ }^{1,3}$

May $\mathrm{Ho}^{2,3}$

Glen D Armstrong 2,3

Daniel A Muruve ${ }^{1,3}$

${ }^{1}$ Department of Medicine

2 Department of Microbiology, Immunology \& Infectious Diseases

${ }^{3}$ Snyder Institute for Chronic Diseases

${ }^{4}$ Leaders in Medicine Program, University of Calgary, Calgary, $\mathrm{AB}$

Introduction: Enterohemorrhagic Escherichia coli (EHEC) is a pathogen that causes hemorrhagic colitis and a severe kidney complication called hemolytic uremic syndrome (EHEC-HUS). EHEC produce Shiga toxins (Stx), which activate cell stress responses, causing inflammation and cell death. NLRP3 is an innate pattern recognition receptor that senses a host of microbial products. In macrophages, activation of NLRP3 causes recruitment of proteolytic proteins to an intracellular complex termed the 'inflammasome', which through caspase- 1 mediates the secretion of proinflammatory cytokine IL-1 and cell death. Clinical studies have demonstrated elevated IL-1 in the serum of HUS patients. Therefore we hypothesized NLRP3 regulates Stx-induced inflammation and kidney injury.

Methods and results: Human monocytic cell line THP1 cells were differentiated into macrophages and treated with Stx. Treatment with Stx resulted in caspase-1 activation, secretion of IL- 1 and cell death (measured by Western blot, ELISA and a lactate dehydrogenase assay), which was abolished with the use of NLRP3 and caspase-1 inhibitors. Immunofluorescent labelling of NLRP3 and ASC in Stx-treated macrophages also revealed inflammasome activation. NLRP3-/- THP1 cells, generated using CRISPR-Cas9 technology, abolished caspase-1 activation, inflammasome formation, IL-1 secretion and cell death, following treatment with Stx. Renal tubular epithelial cells (TEC) underwent cell death after exposure to Stx as visualized by real time live cell imaging of Annexin- $\mathrm{V}$ and propidium iodide labeled cells. Analysis of cell death mediators by Western blot revealed Stx induced caspase-8, 7,3 and PARP activation in renal TEC, which was attenuated in NLRP3-/TEC.
Conclusion: Our studies have established a dual role for NLRP3, which drives inflammation and tissue injury in EHEC-HUS. In macrophages, Stx activated the NLRP3 inflammasome leading to secretion of pro-inflammatory IL-1 $\beta$ and cell death. In addition, exposure to Stx induced NLRP3-mediated cytotoxicity in renal TEC, resulting in apoptosis. As inflammation and tissue injury may regulate the severity of EHEC infection, resulting in HUS, therapeutic strategies that target NLRP3 may be beneficial in reducing morbidity and mortality associated with this devastating disease.

\section{Stage-specific roles of MYSM1 in B cell-mediated immunity}

Kyo Farrington

Michael Förster,

Jessica C. Petrov

Anastasia Nijnik

Department of Physiology and Complex Traits Group, McGill University, Montreal, QC

Introduction: MYSM1 is a chromatin-modifying enzyme with catalytic activity that deubiquitinates histone H2AK119. Its activity is associated with transcriptional activation of various developmental regulators and it is critical for maintaining bone marrow function and the stability of hematopoietic stem cells. Rare mutations in MYSM1 in humans result in lymphopenia with reduced B-cell numbers in circulation. Similarly, Mysml ablation in mice causes a reduction in the numbers of common lymphoid progenitor cells and a severe arrest in B-cell development from the earliest pre-pro B-cell stage of their differentiation. This study aimed to identify whether MYSM1 activity is required at later checkpoints in B-cell development or for B-cell effector functions in humoral immune responses.

Methods: We characterized three conditional Mysm 1-knockout mouse lines: $M y s m 1^{\mathrm{fl} / \mathrm{fl}} \mathrm{mbl-Cre}$, $M y s m 1^{\mathrm{f} / / \mathrm{fl}} \mathrm{CD} 19-\mathrm{Cre}$ and $M y s m 1^{\mathrm{f} / \mathrm{fl}} \mathrm{CD} 21-C r e$, which ablate Mysm I at the pre-pro B, pro-B and transitional stages of B-cell development, respectively. We compared the phenotypes of the three lines to phenotypically wild type mice to assess the role of MYSM1 in B cell differentiation.

Results: $M y s m 1^{\mathrm{f} / \mathrm{l}} \mathrm{mb} 1$-Cre mice showed a 2-fold reduction in the frequency of B-cells in the bone marrow and secondary lymphoid organs, accompanied by increased B-cell activation 
and immunoglobulin class switching, and decreased B-cell survival and proliferation. Mysm $1^{\mathrm{fl} / \mathrm{fl}} \mathrm{CD} 19-\mathrm{Cre}$ and Mysm $1^{\mathrm{f} / \mathrm{f}} \mathrm{CD} 21$-Cre mice were largely normal; showing no alterations in B cell numbers and largely normal B cell responses to in vitro stimulation, except for a minor proliferative defect in the $M y s m 1^{\mathrm{f} / \mathrm{f} l} \mathrm{CD} 19$-Creline.

Conclusions: These findings highlight a critical role for MYSM1 at the pre-pro B stage of development, but show that its activity is largely dispensable from the pro-B cell stage and onwards in their maturation process. Furthermore, our data shows that MYSM1 activity at the pre-pro-B cell stage of development was required for the normal programming of B-cells to respond to antigen stimulation once they have completed their maturation.

\section{Intravitreal Anti-VEGF treatment of myopic choroidal neovascularization: Visual acuity improves when administered prior to the development of fibrosis or atrophy}

Daniel R. Moroz ${ }^{1}$

Monique Munro 2

Michael Fielden ${ }^{2,3}$

1 Cumming School of Medicine, University of Calgary, Calgary, $\mathrm{AB}$

2 Division of Ophthalmology, University of Calgary, Calgary, $\mathrm{AB}$

${ }^{3}$ Calgary Retina Consultants, Calgary, $\mathrm{AB}$

Backgroud: Choroidal Neovascularization (CNV) is a common vision-threatening complication of pathological myopia, yet there is still a need for further consensus on prognostication and clinical management recommendations. Anti-vascular endothelial growth factor (anti-VEGF) agents have ascended as a safe and effective therapy by achieving superior visual acuity gains and quality of life increases as compared with photodynamic therapy.

Purpose: The purpose of this study is to determine if intravitreal ranibizumab or bevacizumab treatment is more effective in improving visual and anatomical outcomes if administered prior to the fibrotic or atrophic stages of myopic CNV.

Methods: A retrospective analysis of twenty-three eyes in as many patients with new-onset subfoveal CNV was performed. Only patients without concurrent age-related macular degeneration and with a spherical equivalent of at least -6.0 diopters were included in the study. All eyes received either intravitreal ranibizumab or bevacizumab injections as the primary treatment on a pro re nata basis for one year. Patient demographics, number of intravitreal injections, duration of treatment, as well as changes in pre- and post-operative OCT characteristics, best-corrected visual acuity (BCVA) and central retinal thickness (CRT) were recorded.

Results: The mean number of ranibizumab (two eyes) or bevacizumab (21 eyes) injections was $3.1 \pm 0.3$ over a mean follow-up time of 14 months. The mean pre-injection BCVA $\log$ MAR was $0.69 \pm 0.31$ and the mean post-injection BCVA was $0.68 \pm 0.30(p=0.49)$. The BCVA for those patients without evidence of fibrosis or atrophy on OCT (17 eyes) improved by $-0.14 \pm 0.09$. In contrast, the BCVA in those with fibrosis or atrophy on OCT ( 6 eyes) worsened by $0.24 \pm$ 0.18 ( $\mathrm{p}=0.048)$. The CRT for those patients without evidence of fibrosis or atrophy decreased by $45.6 \pm 15.7 \mu \mathrm{m}$; however, the CRT for those with fibrosis or atrophy declined by $5.00 \pm 34.6 \mu \mathrm{m}(\mathrm{p}=0.17)$.

Conclusion: Intravitreal anti-vascular endothelial growth factor treatment in eyes with myopic CNV was significantly more effective if administered prior to the fibrotic or atrophic stages of disease progression. This underscores the importance of diagnosing and treating myopic $\mathrm{CNV}$ as early as possible.

\section{NLRP6 in renal injury and inflammation}

Jaye M. Platnich

Hyunjae Chung

N. Adom Bondzi-Simpson

O. Arthur Lau

Justin Chun

Daniel A. Muruve

Department of Medicine, University of Calgary, Calgary, AB

Introduction: Chronic kidney disease (CKD) is a major cause of morbidity and mortality in the general population, affecting over three million Canadians. Regardless of etiology, the progression of CKD involves the recruitment of pro-inflammatory immune cells, the development of renal scarring and, ultimately, kidney failure. The molecular mechanisms that underlie these processes remain poorly understood. The Nod-like receptors (NLRs) are a family of innate immune proteins known to mediate inflammation in various chronic inflammatory diseases, including kidney disease. NLRP6, a recently characterized member of this family, is highly expressed in kidney tissue and is known to 
modulate inflammation in murine models of inflammatory bowel disease and infection. Based on these facts, we hypothesized that NLRP6 regulates both tubulointerstitial inflammation and fibrosis in the context of chronic kidney disease.

Methods and results: The expression of NLRP6 mRNA was characterized in murine and human tissues under both basal conditions and disease states using quantitative polymerase chain reaction (qPCR). A panel of murine organs was examined, with the highest expressing organs found to be intestine, liver, and kidney. NLRP6 is also highly expressed in human kidney. The expression was found to be highest in the glomerular subfraction of both species. High levels of expression were also found in human polymorphonuclear cells (PMNs).

Kidney injury was studied in both wild type and NLRP6-deficient mice via the unilateral ureteral obstruction (UUO) model of disease. Briefly, the left ureter of a cohort of mice was ligated with a suture in order to generate a post-renal, obstructive model of kidney injury. Following seven- and 14-day time points, the mice were sacrificed, both kidneys were harvested, and samples were taken for histology, qPCR, and Western blotting. Specifically, the samples were examined for markers of inflammation (e.g. CD11b) and fibrosis (e.g., TGF, SMA and CTGF). qPCR analysis revealed a significant drop in NLRP6 mRNA following UUO, compared with controls that underwent sham procedures. Histology and Western blot analysis revealed no difference in phenotype between wild type and NLRP6-deficient animals in terms of inflammation or fibrosis.

Conclusions: Our studies have revealed NLRP6 to be highly expressed in both murine and human kidney tissue, particularly within the glomerular subfraction. This expression was differentially expressed in the context of disease, with expression decreasing substantially following the UUO model of murine kidney disease. No difference in disease severity was noted between the wildtype and NLRP6- deficient animals. Based on the glomerular expression of the mRNA, we concluded that UUO was the incorrect disease model to study in the context of this gene and that NLRP6 plays no significant role in mediating tubulointerstitial inflammation and fibrosis in an obstructive model of kidney injury.

\section{Phenylboronic acid-based polymeric micelles for mucoadhesive anterior segment ocular drug delivery}

Graeme Prosperi-Porta ${ }^{1}$

Stephanie Kedzior ${ }^{2}$

Benjamin Muirhead ${ }^{1}$

Heather Sheardown ${ }^{1,2}$

${ }^{1}$ School of Biomedical Engineering

${ }^{2}$ Department of Chemical Engineering, McMaster University, Hamilton, ON

Introduction: Topical administration of ocular pharmaceuticals is most commonly used, due to its ease of application, low physician involvement and minimal invasiveness. Unfortunately, topical ocular drug delivery is extremely inefficient due to the natural barriers such as the impermeable cornea and sclera, and the rapid tear turnover that rinses the drug off of the ocular surface. These precorneal clearance mechanisms result in ineffective drug penetration, requiring frequent administration that results in high systemic exposure and potential for side effects.

Purpose: This work is aimed at improving the precorneal bioavailability of cyclosporine A (CycA) for the treatment of dry eye disease using a polymeric mucoadhesive micelle delivery system.

Methods: Poly(D,L-lactide)-b-poly(methacrylic acid-co-3-acrylamidophenylboronic acid) copolymers were synthesized using reversible addition-fragmentation chain transfer polymerization with varying compositions of 3-acrylamidophenylboronic acid (PBA) from 0-30 mol\%. Blank and CycA loaded micelles were formed using the precipitation method from acetone into purified water. Micelle size was determined using dynamic light scattering (DLS), and morphology was confirmed with transmission electron microscopy (TEM). In vitro mucoadhesion was studied using surface plasmon resonance after adsorbing type III porcine stomach mucin to gold sensors. In vitro release from CycA loaded micelles was measured using high performance liquid chromatography. In vitro cell viability was measured using human corneal epithelial cells (HCEC) at 5 $\mathrm{mg} \mathrm{mL}^{-1}$ and $1 \mathrm{mg} \mathrm{mL}^{-1}$ micelles after 24 and 72 hours. In vivo ocular irritation was examined by applying $10 \mu \mathrm{L}$ of $5 \mathrm{mg} \mathrm{mL}^{-1}$ micelle solution daily into the left eye of Sprague-Dawley rats. A modified Draize protocol with slit lamp microscopy and fluorescein eye stain was used to assess the left and contralateral eyes after 10 days.

Results: CycA-loaded micelles formed nearly transparent solutions when PBA content was $\leq 10 \mathrm{~mol} \%$, but formed 
stable turbid suspensions when PBA content was $\geq 20 \mathrm{~mol} \%$. DLS showed micelle diameters ranging from 35 to $64 \mathrm{~nm}$ as PBA composition increased from 0 to $30 \mathrm{~mol} \%$, respectively. CycA loading did not alter micelle diameter for PBA compositions $\leq 10 \mathrm{~mol} \%$ ( $p>0.05)$, but were found to significantly reduce micelle diameter when PBA content was $\geq$ $20 \mathrm{~mol} \%(\mathrm{p}<0.05)$. TEM confirmed round morphologies indicative of micelles. Mucoadhesion increased with higher PBA content, but this effect diminished at compositions $>10$ mol\% PBA. CycA loading was $>99 \%$ for all micelles with approximately $40 \%$ released after 48 hours and up to $80 \%$ released after 14 days. The micelles were not found to be cytotoxic to HCECs at either one or $5 \mathrm{mg} \mathrm{mL}^{-1}$ at 24 or 72 hours, but did reduce proliferation and altered cell morphology compared with controls. Ocular irritation tests showed minimal corneal irritation after 10 days of exposure, suggesting good biocompatibility.

Conclusion: This study shows that mucoadhesive biomaterials have significant potential to improve ocular drug delivery by increasing the precorneal bioavailability of pharmaceuticals. Overall, these micelles show potential to reduce dosage, frequency and systemic side effects due to their mucoadhesive properties allowing for targeted drug delivery to the ocular surface. Future studies will examine corneal bioavailability and drug penetration into the anterior chamber.

\section{The immunomodulatory role of marcrophage on hair follicle stem cells after a full thickness skin injury}

Waleed Rahman
Yunan Liu $^{1}$
Eko Raharjo $^{1}$
Jessica Yoon
Sarthak Sinha $^{1}$
Robin Yates $^{1}$
Paul I.Kubes
Jeff Biernaskie

1 Department of Comparative Biology and Experimental Medicine, Faculty of Veterinary Medicine

2 Snyder Institute for Chronic Diseases, Cumming School of Medicine, University of Calgary, Calgary, $\mathrm{AB}$

Introduction: The hair follicle cycle (anagen, catagen and telogen) stands as the most impressive example of scarless regeneration in mammalian biology. Our lab is interested in understanding how local and systemic signals modulate the activity of hair follicle stem cells and the continuous regeneration of hair follicles. After a full-thickness wound, surrounding dormant hair follicles regenerate to produce a new hair fibre, a process known as wound-induced hair growth (WIHG). The cellular and molecular mechanisms underlying this process remain a mystery. One study did show that without wound macrophage WIHG is blocked; however, the role of macrophage in WIHG, their identity, origin and functional diversity, and factors mediating hair follicle stem cell activation remain unknown. We hypothesize that monocytes infiltrate the wound site, transition into anti-inflammatory macrophage, and secrete a factor that activates hair follicle stem cells and hairgrowth.

Methods: To address this question, we used various transgenic mice to genetically ablate and fluorescently trace monocytes, macrophage, dendritic cells and mast cells over the course of wound healing. We used flow cytometry, confocal microscopy and gross skin observations to measure and visualize the effects of cell depletion on the hair growth after wounding.

Results: First, ablation of CD11 $b^{+} \mathrm{CD} 11 \mathrm{c}^{-}$macrophage 7-11 days post injury (dpi) completely blocked WIHG. Loss of and not $\mathrm{CD} 11 \mathrm{~b}^{\mathrm{lo}} \mathrm{CD} 11 \mathrm{c}^{\mathrm{hi}}$ dendritic cells and mast cells during wound healing did not block WIHG. Next, we created a temporal and subtype-specific profile of wound-associated macrophage using a dual-reporter mouse, CCR2RFP/+* CX3CR1GFP/+. With flow cytometry, the various expression levels of CCR2 and CX3CR1 fit along a red, orange, yellow, lime and green color gradient and correspond to the various macrophage/monocyte phenotypes present in the wound. Interestingly, fluorescently-labelled cells were transitioned from an orange phenotype (CCR $2>C X 3 C R 1$ : pro-inflammatory) in the early wound ( 12 hours $-6 \mathrm{dpi}$ ) to a lime phenotype (CCR2<CX3CR1: anti-inflammatory) in the late wound (6-10 dpi). This shift coincides with hair follicle stem cell activation preceding WIHG. Furthermore, the phenotype switch and WIHG was non-existent in CX3CR1-deficient mice.

Conclusion: Altogether, preliminary data suggests that CX3CR1 hiCCR2 lo CD11 ${ }^{+} \mathrm{CD} 11 \mathrm{c}$ macrophages stimulated hair follicle regeneration in a CX3CR1-dependant manner after a full-thickness excision wound. Understanding the role macrophages have in hair follicle regeneration will provide us with a novel therapeutically target to treat various forms of alopecia. Furthermore, our data suggests that macrophages are an important part of the hair follicle stem cell niche. 


\section{Anti-cryptococcal recognition and signalling in Natural Killer (NK) cells}

\author{
Richard F. Xiang1,2 \\ Danuta Stack ${ }^{1,2}$ \\ Shu Shun $\mathrm{Li}^{1,2}$ \\ Shaunna M. Huston ${ }^{1,2}$ \\ Henry Ogbomo ${ }^{1,2}$ \\ Stephen K. Kyei ${ }^{1,2}$, \\ Christopher H. Mody ${ }^{1,2,3}$
}

${ }^{1}$ Department of Microbiology, Immunology and Infectious Diseases

2 Snyder Institute for Chronic Disease

3 Department of Internal Medicine, University of Calgary, Calgary, $\mathrm{AB}$

Introduction: Cryptococcus is a pathogen that causes fatal meningitis and pneumonia by subverting the host's adaptive immune response. Fortunately, NK cells of the innate immune system are capable of killing Cryptococcus. NK cytotoxicity against tumor and virally-infected targets are well studied, but less is known about anti-cryptococcal signalling. In this study, we investigated the role of Rac, Src family kinases (SFK) and integrins in anti-cryptococcal cytotoxicity.

Methods: The involvement of Rac, SFK, integrin- linked kinase (ILK), beta-1 and beta-2 integrins and NKp30 were investigated using a combination of small molecule inhibitors, siRNA knockdown, activated Rac pull down assays and immunoblots: Rac function was inhibited by EHT1864, Rac inhibitor II, and small interference RNA (siRNA); SFK were inhibited by dasatinib. ILK was inhibited by Cpd 22; Beta-1 and 2 integrins were inhibited by siRNA knockdown; and NKp30 was inhibited by inhibitory antibodies.

Results: Our studies found that Cryptococcus activated both SFK and Rac signalling pathways. SFK were found to be an activator of the PI3K $\rightarrow$ Erk cytotoxicity pathway. Surprisingly, Rac also functioned as a non-canonical activator of PI3K, and that individually Rac and SFK were not sufficient for activation of PI3K. Additional studies revealed that Rac was activated by beta- 1 integrins through an ILK pathway. On the other hand, SFK were activated by the NK cytotoxicity receptor, NKp30.

Conclusion: Our findings build a model of NK cell mediated anti-fungal killing that is initiated by integrin and NKp30 activation of Rac and SFK pathways respectively. The Rac and SFK pathways ultimately converge on a central PI $3 \mathrm{~K} \rightarrow$ Erk cytotoxicity pathway. Our model provides various potential therapeutic targets that could enhance cryptococcal clearance mediated by the innate immune system.

\section{Muscle fascicle length is reduced in old age owing to shorter sarcomeres and serial sarcomere loss}

Sean Crooks

Geoffrey A. Power

Walter Herzog

Human Performance Laboratory, University of Calgary, Calgary, AB

Introduction: With natural human aging, there are alterations to the structural components of the muscular system that result in impaired contractile function and decreased performance in activities of daily life. Despite reductions in active force, passive force is often reported to be greater in muscles from older adults compared with younger adults. A potential unexplored mechanism for increased passive force in old age could be decreased fascicle lengths owing to a decrease in sarcomeres number in series.

Purpose: The purpose of this study was to compare muscles of young and old rats to determine fascicle length, sarcomere numbers and the sarcomere length at which peak force is obtained (i.e., plateau of the force-length (FL) relationship; $L_{o}$ ). It was hypothesized that fascicle length would be shorter owing to fewer sarcomeres in series.

Methods: A young ( 20 human years) $(\mathrm{n}=9)$ and old cohort ( $~ 75-80$ human years) $(n=8)$ of rats were used. The medial gastrocnemius muscle (MG) from the right leg was surgically isolated and attached to a force transducer. For muscle activation, the tibial nerve was electrically stimulated and $L_{o}$ was determined by performing a standard FL relationship. The animals were sacrificed and the MG muscle was fixed at $\mathrm{L}_{\mathrm{o}}$. Next, the muscles were digested in acid and fascicles isolated for sarcomere length measurement by laser diffraction. Fascicle length measurements were taken using pixel counting software. Serial sarcomere numbers were calculated by dividing the fascicle length by the average sarcomere length. In total, 340 fascicle length and 1,700 sarcomere length measurements weretaken.

Results: A reduction in fascicle length of $\sim 14 \%$ was observed in the old rats when compared with the young rats, $\mathrm{p}<0.001$. There was a reduction in average sarcomere length of $\sim 4 \%$ in the old rats versus the young rats, $\mathrm{p}<0.001$. The MG of old 
rats showed $\sim 10 \%$ fewer sarcomeres in series when compared with young rats, $\mathrm{p}<0.001$.

Conclusion: The reduction in fascicle length was associated with a lower sarcomere number in series for the old age cohort. Serial sarcomere number is important when considering the FL relationship; for example, if the limbs of young and old were at matched joint angles such as what occurs in vivo, the shorter fascicles in the old would suggest the sarcomeres would be stretched to longer lengths. This would mean that older adults may be operating further on the descending limb of the FL relationship contributing to muscle weakness. Additionally, as sarcomeres in aged muscle are being pulled to longer lengths, passive force producing elements such as titin may play a larger role in the increased passive tension in older adult muscle.

The results of this study were consistent with the hypothesis that in muscle fascicles from aged muscle, not only are sarcomeres lost in parallel, but also in series, resulting in shorter fascicles. But quite unexpectedly, average sarcomere length was shorter at $\mathrm{L}_{\mathrm{o}}$ in the old rats compared with the young rats. These results may help to explain increased stiffness and passive force of muscles of olderindividuals.

\section{Effects of concentration on synergistic hyaluronan-PRG4 cartilage boundary lubrication}

Miles Hunter

Tannin Schmidt

Faculty of Kinesiology, University of Calgary, Calgary, $\mathrm{AB}$

Introduction: Proteoglycan 4 (PRG4) and hyaluronan (HA) are constituents of synovial fluid (SF) that act synergistically to contribute to the boundary lubrication of articular cartilage in a dose-dependent manner. However, the potential concentration dependency of this HA-PRG4 synergism remains to be elucidated. The objective of this study was therefore to evaluate the in vitro cartilage boundary lubricating ability of PRG4+HA at varying concentrations of each.

Methods: Cartilage boundary lubricating ability was assessed using bovine osteochondral samples in a cartilage-on-cartilage friction test, as previously described. Test sequences were as follows: Test 1 (PRG4 dose response, + constant $\mathrm{HA}=3.33$ $\mathrm{mg} / \mathrm{mL}, \mathrm{n}=6$ ): phosphate-buffered saline (PBS), $150 \mu \mathrm{g} / \mathrm{mL}$ PRG4 + HA, 450 $\mathrm{g} / \mathrm{mL}$ PRG4 + HA, $1500 \mu \mathrm{g} / \mathrm{mL}$ PRG4 + HA, SF; and Test 2 (HA dose response, + constant PRG4 = $450 \mu \mathrm{g} / \mathrm{mL}, \mathrm{n}=5):$ PBS, $0.3 \mathrm{mg} / \mathrm{mL}$ HA + PRG4, $1.0 \mathrm{mg} / \mathrm{mL}$ HA + PRG4, $3.33 \mathrm{mg} / \mathrm{mL}$ HA + PRG4, SF. Static,
ustatic,Neq, and kinetic, < $\mu$ kinetic,Neq $>$, friction coefficients were then calculated.

Results: In all tests, $\mu$ static,Neq values were consistently highest in PBS and lowest in SF, with all PRG4+HA combinations tested being similar to SF. Test 1: $<\mu$ kinetic,Neq> values in varying PRG4 concentrations + constant HA were neither significantly different from each other nor from SF. Test 2: < $\mu$ kinetic,Neq > values in varying HA concentrations + constant PRG4 were neither significantly different from each other nor from SF.

Conclusions: These results demonstrated that HA+PRG4 lubrication synergism was maintained, provided that either PRG4 or HA was present at a physiologically normal concentration, and that these combinations provided lubricating ability approaching that of SF. Intra-articular PRG4 has been shown to be chondroprotective in animal injury models of osteoarthritis; therefore, clarifying the PRG4+HA synergism will contribute to the potential application of PRG4, with or without HA, as an improved biotherapeutic treatment.

\section{Long term in vivo kinematics of the ovine stifle joint following anterior cruciate ligament transection}

K.I. Barton
J.L. Norman
B. Heard
M. Shekarforoush
M. Atarod
J. Sevick
Y. Achari
C.B. Frank
D.A. Hart
N.G. Shrive

University of Calgary, Calgary, AB

Introduction: Osteoarthritis $(\mathrm{OA})$ is a degenerative joint disease involving the breakdown of articular cartilage, which is common after injury or with aging. Following knee injury, sheep develop OA at a slightly accelerated rate compared with humans but with some similar patterns. In an ovine knee injury model, altered gait mechanics and degradation of the cartilage have been observed 20 weeks post anterior cruciate ligament (ACL) transection (Tx) surgery.

Purpose: Potential alterations in gait at 40 weeks post ACL Tx surgery remain to be determined; therefore, the objective of 
this study was to investigate the in vivo kinematics of the ovine stifle joint over time following ACL Tx.

Methods: Skeletally mature 3- to 4-year-old female Suffolk-cross sheep had a bone plate implanted onto each of the proximomedial aspect of the tibia and the distolateral aspect of the femur of their right hind limb, four weeks prior to kinematic testing. A novel tibial post was designed to further refine accurate kinematic measurement. To assess if plate implantation and/or ACL Tx reduced hind limb vertical ground reaction force (VGRF), sheep were led across an embedded force platform until 20 hind limb hoof strikes were recorded at 1,200 Hz. As described previously, on the day of kinematic testing, a stainless steel post was attached to each plate and an instrumented spatial linkage (ISL) mounted between them. The ISL consists of six rotational encoders providing six degrees of freedom (6-DOF) to its motion. The 6-DOF in vivo kinematics of the stifle joint were measured during "normal gait" while the sheep walked on a treadmill at 2 $\mathrm{mph}(0.89 \mathrm{~m} / \mathrm{s})$.

Each sheep then underwent arthroscopic surgery on their right hind limb in which their ACL was transected mid-substance. The in vivo gait kinematics were measured again over time, at 20-22 weeks and 40 weeks following ACL Tx. Following kinematic testing at 40 weeks, the animals were euthanized by intravenous injection and their hind limbs were disarticulated. All surrounding tissue was removed from the joint. A coordinate measuring machine was used to measure anatomic landmarks on the bone with respect to the ISL in order to create an anatomically relevant coordinate system. Throughout tissue dissection, gross morphological grading was conducted by one expert observer for gross defects, osteophyte formation, and meniscal damage.

Results: The maximum VGRF in the hind limbs within each animal demonstrated different patterns over time. In all animals, there was a shift from intact to the short-term time point (20-22 weeks) in the flexion- extension component. In Animals 2 and 3, the patterns continued to get worse over time; however in Animal 1 the flexion-extension component returned to a more normal pattern. In the medial-lateral component, there was a considerable shift from intact in the short term (20-22 weeks) in all three animals, which continued to get worse over time (at 40 weeks). In all sheep, the inferior-superior and internal-external components were shifted at 20-22 weeks, but returned to more normal patterns by 40 weeks. Each animal displayed variability in changes from the intact kinematic measurement. The combined gross morphological score in all ACL Tx operated limbs was higher $(p=0.0545)$ than that of the respective contralateral limbs.
Conclusion: The most striking finding of this study was the considerable inter-subject variability in both the force plate testing and in the in vivo kinematics. In addition, there was a large shift from intact in the medial-lateral component in the short-term (20-22 weeks, not significant in previous studies) and long-term ( 40 weeks).

\section{Voluntary physical exercise promotes remyelination in a mouse model of multiple sclerosis}

Samuel K. Jensen ${ }^{1}$

Michael B. Keough ${ }^{1}$

Nathan Michaels ${ }^{1}$

V. Wee Yong ${ }^{1,2}$

${ }^{1}$ Department of Clinical Neurosciences

2 Department of Oncology, Hotchkiss Brain Institute at University of Calgary, Calgary, $\mathrm{AB}$

Introduction: Multiple sclerosis (MS) is a common immune-mediated disease of the central nervous system (CNS) characterized by the presence of multifocal lesions with demyelination and axonal injury. Many of the reactive changes that occur following plaque formation, such as an altered lesion microenvironment, persist and ultimately impair attempts at regenerative processes, including remyelination. Currently available treatments for MS focus on preventing leukocytes from entering the CNS or suppressing their activity, with the goal of halting new lesion formation; however, they do not address the chronic failure to repair lesion-induced damage that contributes to progressive disability. Remyelination therapies that attempt to address this latter element, aiming to reverse past demyelination in order to restore homeostatic axon conduction and prevent future axonal injury, are strongly desired by the MS population. Physical activity is known to impact wellbeing in MS with a number of beneficial effects in neurodegenerative disorders, leading us to address whether exercise has the capacity to improve remyelination.

Methods: The ability of physical exercise to promote remyelination was investigated. We induced a focal demyelinating lesion via injection of lysophosphatidylcholine (LPC) into the ventrolateral white matter of the murine spinal cord. Mice were housed singly and given free access to an electronically-monitored running wheel on the day of injury until sacrifice at either seven or 14 days post-lesion (representing peak demyelination and early remyelination, 
respectively). Conditions were mirrored in control animals, with the exception of the wheel being locked.

Results: We have found that LPC-lesioned mice allowed free access immediately post-injury to a running wheel possess $35.9 \%$ more mature oligodendrocytes within the lesion; a $47.6 \%$ increase in lesion-associated myelin basic protein and a 2.7-fold increase in lesion-associated remyelinated axons at 14 days post-lesion, when compared with demyelinated animals housed with a locked wheel. Furthermore, when comparing remyelinated axons between the two groups, the axons within exercising animals possess significantly thicker myelin, as noted by a decreased g-ratio (the ratio between axonal diameter and myelin diameter). Impressively, at 14 days post-lesion only $12.9 \%$ of axons remained demyelinated in the exercising group compared with $51.3 \%$ of axons in the sedentary controls.

Conclusions: We demonstrated that physical exercise was efficacious in promoting the remyelination of experimental toxin-mediated demyelination. This was the first conclusive demonstration of a disease-modifying effect of exercise relevant to MS that is independent of the modulation of secondary disease outcomes. Further, the work provides a foundation for mechanistic investigations and a rationale for further study of exercise in MS patients.

\section{The use of 3D gait analysis in the development of exercise-based interventions in hip osteoarthritis: Does it improve outcomes compared with a standard clinical assessment? A pilot RCT}

RJ Leigh ${ }^{1,2}$

ST Osis ${ }^{2}$

R Ferber $^{2}$

${ }^{1}$ Faculty of Medicine, Leaders in Medicine Program

${ }^{2}$ Faculty of Kinesiology, University of Calgary, Calgary, $\mathrm{AB}$

Background: Effective non-pharmacological and non-surgical treatments are needed in the management of hip osteoarthritis (OA). To date, the effect size of exercise as an intervention in hip OA remains small. This small effect size may in part be related to the difficulty in precisely identifying the impairments inherent to hip OA patients. 3D gait analysis (3DGA) is thought to be a precise, quantitative tool that can identify subtle biomechanical changes in the movement patterns of both healthy and diseased populations. Whether 3DGA is an effective tool in the development of exercise interventions and subsequent outcomes in hip OA patients remains unknown.

Methods:

Design and participants: The study was an assessor- and participant-blinded pilot randomized controlled trial involving subjects with clinical and radiographic diagnosis of hip osteoarthritis. Intervention: Subjects with hip OA $(\mathrm{n}=40)$ were randomized to two groups and received an exercise intervention (strengthening, ROM) based on the results of a 3D gait analysis (GATE group; $\mathrm{n}=20$ ) or based on the results of a standard clinical assessment (CLIN group; $n=20$ ). The $3 \mathrm{D}$ gait analysis assessment measured lower extremity kinematic joint angles during treadmill walking $(1.1 \mathrm{~m} / \mathrm{s})$, while the standard clinical assessment measured ROM, flexibility, strength and alignment measures.

Primary outcome measures: Hip pain and hip function was measured according to the Western Ontario McMaster Universities Osteoarthritis Index (WOMAC) at eight weeks follow-up.

Analysis: Between group differences in WOMAC pain and function were assessed using an analysis of covariance (ANCOVA) with significance at $\mathrm{P}<0.05$.

Results: There were no significant differences in WOMAC pain noted between the GATE and CLIN groups at eight weeks follow-up, when controlling for baseline pain $(F(1,26)=$ 3.646, $\mathrm{P}=0.07)$. Similarly, no significant differences in WOMAC function were noted between groups $(\mathrm{F}(1,26)=$ 3.235, $\mathrm{P}=0.08)$ after controlling for baseline function. No significant differences were noted between groups for any of the secondary outcomes including quality of life or activities of daily living.

Conclusions: Developing exercise programs using 3D gait analysis did not result in improved outcomes in hip OA patients when compared with a standard clinical assessment. Within this population, 3D gait analysis may best be suited for identifying biomechanical abnormalities rather than for prescribing exercise. 
Acute exposure to volatile anesthetics impairs synaptic, but not cytoskeletal, architecture

\author{
Ryden Armstrong ${ }^{1,2}$ \\ Fenglian $\mathrm{Xu}^{2}$ \\ Daniela Urrego ${ }^{1,2}$ \\ Munir Qazzaz ${ }^{2}$ \\ Mario Pehar ${ }^{3}$ \\ J.N. Armstrong 3 \\ Naweed I. Syed ${ }^{2}$ \\ ${ }^{1}$ Leaders in Medicine \\ ${ }^{2}$ Hotchkiss Brain Institute \\ 3 Department of Anesthesia, Cumming School of Medicine, \\ University of Calgary, Calgary, $\mathrm{AB}$
}

Introduction: Recent pre-clinical and clinical studies have questioned the long-term safety of volatile anesthetics when administered to young children during surgery. Pre-clinical animal studies have demonstrated that anesthesia exposure during critical periods of neurodevelopment leads to behavioral and cognitive impairments. Retrospective clinical trials have shown that children that have been exposed to general anesthesia have an increased risk of developing learning disabilities in later life; however, the mechanisms that lead to these impairments remain unknown. Our work aims to determine the subcellular targets that are impacted by acute anesthetic exposure to clinically relevant doses of the two most commonly used volatile anesthetics: sevoflurane; and desflurane.

Methods: Rat cortical neurons were isolated from post-natal day 0 rats, and exposed to either $1.3 \%$ sevoflurane or $4.3 \%$ desflurane for one hour in an airtight chamber. Cells were then stained for cell viability, cytoskeletal and synaptic proteins and imaged with confocal microscopy.

Results: Cell viability was not impacted by either sevoflurane or desflurane, which implies that these drugs may exert sub-lethal effects on neuron function. To investigate this, we then visualized cytoskeletal $\mathrm{F}$-actin and $\beta$-tubulin with confocal microscopy and observed no changes in neurite branching or total cytoskeletal protein expression. Finally, we stained neurons for the pre- and post-synaptic proteins, synaptophysin and PSD-95, respectively. Confocal microscopy demonstrated that both sevoflurane and desflurane led to a reduction in co-localization of these proteins, indicating a reduction in functionalsynapses.

Conclusions: Our study demonstrated that at clinically relevant doses, sevoflurane and desflurane exerted sub-lethal impacts on rat cortical neurons. Neither sevoflurane nor desflurane reduced cell viability or the development of neurites and a robust cytoskeleton; however, the co-localization of synaptic proteins was dramatically reduced. As such, synaptic transmission may be impaired. Following the "fire-together, wire-together" paradigm, this impairment of synaptic transmission may then lead to downstream impairments in cognitive function in later life.

\section{Cerebral arterioles can dilate to synaptic activation without astrocyte endfoot $\mathrm{Ca} 2+$ transients}

Ádám Institoris

David G. Rosenegger

Grant R. Gordon

Hotchkiss Brain Institute, Department of Physiology and Pharmacology, University of Calgary, Calgary, AB

Introduction: $\mathrm{Ca}^{2+-}$ dependent pathways in neurons and astrocyte endfeet initiate changes in arteriole diameter to regulate local brain blood flow. Whether there exists a threshold of synaptic activity in which arteriole diameter is controlled independent of astrocyte endfeet $\mathrm{Ca}^{2+}$ remains unclear.

Methods: Two-photon fluorescence microscopy was used to examine synaptically-evoked synthetic or genetic $\mathrm{Ca}^{2+}$ indicator signals around penetrating arterioles in acute slices of the rat neocortex.

Results: We discovered a threshold below which vasodilation occurred in the absence of endfeet $\mathrm{Ca}^{2+}$ signals but with consistent neuronal $\mathrm{Ca}^{2+}$ transients.

Conclusion: This suggests that endfoot $\mathrm{Ca}^{2+}$ is not necessary for activity-dependent vasodilation under subtle degrees of brain activation. 


\section{The critical role of microglial $\mathrm{P} 2 \mathrm{X} 7$ receptors in the development of morphine tolerance}

\author{
Heather Leduc-Pessah ${ }^{1,3}$ \\ Nicholas Weilinger ${ }^{2,3}$ \\ Churmy Fan ${ }^{1,3}$ \\ Nicole Burma ${ }^{1,3}$ \\ Roger Thompson ${ }^{2,3}$ \\ Tuan Trang ${ }^{1,3}$
}

1 Departments of Comparative Biology \& Experimental Medicine, and Physiology \& Pharmacology

${ }^{2}$ Department of Cell Biology \& Anatomy

${ }^{3}$ Hotchkiss Brain Institute, University of Calgary, Calgary, AB

Introduction: Chronic pain is a pervasive clinical problem and profoundly impacts quality of life. Morphine is a potent analgesic used in the treatment of chronic pain; however, its use is limited by the development of analgesic tolerance, such that higher and more frequent doses are required to achieve the same level of pain control. Growing evidence suggests that microglia are critically involved in the development of morphine tolerance. In the present study, we examined the role of microglial ATP-gated P2X7 receptors (P2X7Rs) in the development of morphine analgesic tolerance.

Methods: Male Sprague Dawley rats were treated with morphine $(15 \mathrm{mg} / \mathrm{kg}$; IP) over 7 days. Anti-nociception was assessed using thermal tail flick and mechanical paw pressure tests. Cultured BV2 microglial cells were treated with morphine $(1 \mu \mathrm{M})$, and changes in $\mathrm{P} 2 \mathrm{X} 7 \mathrm{R}$ protein expression were assessed by Western blot, whereas modulation of P2X7R function was determined by whole cell patch clamp recordings and calcium imaging.

Results: Repeated morphine caused a progressive decline in morphine anti-nociception and a loss in morphine analgesic potency. This coincided with an increase in microglial reactivity and an up-regulation of total $\mathrm{P} 2 \mathrm{X} 7 \mathrm{R}$ expression. Intrathecal administration of the selective P2X7R antagonist, A740003, daily with morphine caused a significant attenuation of the development of analgesic tolerance. To further characterize the effects of morphine on microglial P2X7Rs, we used the BV2 microglial cell line and found an up-regulation of total $\mathrm{P} 2 \mathrm{X} 7 \mathrm{R}$ expression with repeated morphine administration consistent with our observation in spinal cord lysates. The morphine-induced increase in P2X7R protein expression was concomitant with a potentiation of BzATP evoked P2X7R calcium responses and inward current. Both the increase in P2X7R expression and potentiation of function were selective to $\mu$ receptor activation. We next tested whether receptor phosphorylation played a role in the potentiation of P2X7R function. Daily co-administration of a protein kinase inhibitor with morphine prevented the morphine-induced potentiation of P2X7R BzATP- evoked calcium influx and inward current. This demonstrated a critical role for morphine-mediated phosphorylation of the P2X7R and, through this mechanism, a modulation of ion channelfunction.

Conclusion: Our findings demonstrated that microglial P2X7Rs were causally involved in the development of morphine analgesic tolerance. In addition, we found that repeated morphine increased total microglial P2X7R expression and induced a potentiation of $\mathrm{P} 2 \mathrm{X} 7 \mathrm{R}$ function. These critical mechanisms involved in the modulation of P2X7R function in response to morphine present a potential therapeutic target in the management of morphine tolerance. Supported by: NSERC, CFI, CIHR, Rita Allen Foundation and AIHS

\section{Sequestration of toxic reactive aldehydes by the monoamine oxidase inhibitor phenelzine and its metabolite-phenylethylidenehydrazine (PEH)}

\author{
DmitriyMatveychuk ${ }^{1}$ \\ Yanlin Wang ${ }^{2}$ \\ Satyabrata $\operatorname{Kar}^{2}$ \\ Glen B. Baker ${ }^{1}$ \\ ${ }^{1}$ Neurochemical Research Unit \\ ${ }^{2}$ Centre for Prions and Protein Folding Diseases, Department \\ of Psychiatry, University of Alberta, Edmonton, $\mathrm{AB}$
}

Introduction: The antidepressant and anxiolytic drug, phenelzine, has been shown in animal models of cerebral ischemia, multiple sclerosis and traumatic brain injury to possess neuroprotective properties. It appears that a metabolite of phenelzine, $\beta$-phenylethylidenehydrazine $(\mathrm{PEH})$, shares some of its neuroprotective mechanisms. Due to the presence of a hydrazine moiety, phenelzine and PEH may be able to directly sequester aldehydes. Levels of acrolein and malondialdehyde, toxic reactive aldehydes generated by lipid peroxidation, are increased in the brains of Alzheimer disease (AD) patients and are implicated in $\mathrm{AD}$ pathogenesis via several pathways, including potentiation of amyloid- $\beta$ oligomerization/fibrillogenesis and tau hyperphosphorylation. We investigated the ability of phenelzine and PEH to sequester acrolein and malondialdehyde in vitro, attenuate 
acrolein toxicity in mouse cortical neurons and reduce rat whole brain levels of extractable acrolein and malondialdehyde exvivo.

Methods: Gas chromatography combined with mass spectrometry (GC-MS) was employed for quantitation of acrolein and malondialdehyde sequestration in vitro and in rat whole brain following administration of both drugs. Mouse cortical neurons were co-treated with acrolein and phenelzine or PEH, followed by assessment of cell viability with the MTT assay.

Results: Phenelzine and PEH dose-dependently sequestered acrolein and malondialdehyde in vitro. In mouse cortical neurons, cell viability was increased when acrolein was co-treated with phenelzine or PEH. Neither phenelzine nor PEH treatment had a significant effect on rat whole brain acrolein levels. Phenelzine, but not PEH, reduced rat whole brain malondialdehyde levels.

Conclusion: These results suggest that further investigation is warranted on aldehyde sequestration by phenelzine and PEH, as both drugs have the potential to be protective against aldehyde toxicity and may be useful as adjunctive treatments in AD.

\section{Amyloid beta modulates opening of pannexin-1 channels during hypoxia}

Laura A. Palmer ${ }^{1,3}$

Roger J. Thompson ${ }^{2,3}$

${ }^{1}$ Department of Neuroscience,

${ }^{2}$ Department of Cell Biology and Anatomy

${ }^{3}$ Hotchkiss Brain Institute, Cumming School of Medicine, University of Calgary, Calgary, $\mathrm{AB}$

Introduction: Ischemic stroke is a condition during which blood flow is reduced in the brain, resulting in downstream hypoxia and cell death. Under low oxygen conditions, there is excessive neurotransmission and impaired neurotransmitter reuptake to astrocytes due to a loss of energy substrates $\left(\mathrm{O}_{2}\right.$ and ATP). This results in excess glutamate receptor stimulation, anoxic depolarizations (aDPs) and excitotoxicity. The aDP is well characterized, and pannexin-1 channels (Panx1) have been demonstrated to be an important contributing factor to ionic dysregulation during the aDP. Stroke survivors are at risk of developing neurodegenerative disorders such as Alzheimer disease (AD). While the reason for this is not fully defined, it has been reported that brain hypoxia leads to increased production of the pathological form of the protein amyloid beta $(A \beta) . A \beta$ is aggregated into neuronal plaques in patients with $A D$, and oligomeric $A \beta$ is thought to disrupt synaptic activity and initiate inflammation pathways, contributing to the common symptoms of the disease.

Interestingly, little is known about the physiological role of $A \beta$, and less still is known of the reason behind its increased production during hypoxia. Our overall hypothesis is that physiological concentrations of $A \beta$ can modulate responses to hypoxia.

Methods: Whole cell patch clamp electrophysiology was used in hippocampal slices from rats, which were continuously perfused with hypoxic artificial cerebral spinal fluid in order to mimic ischemia and induce the aDP. Various concentrations of $\mathrm{A} \beta$ protein ( $\mathrm{pM}$ to $\mu \mathrm{M}$ range) were applied concurrently with hypoxia. Low concentrations of $A \beta$ were applied with a Panxl antibody in order to observe if there are any additive neuroprotective effects of pharmacologically blocking Panx 1 in the presence of $A \beta$. A different model of stroke involving the overstimulation of the $N$-methyl- $D$-aspartate receptor (NMDAR) was used in a separate set of experiments to assess if $A \beta$ can directly interact with Panxl channels. $100 \mu \mathrm{M}$ NMDA was applied to the tissue with and without $A \beta$ in order to determine if it could block currents associated with toxicity and cell death.

Results: Application of low concentrations of $A \beta$ during hypoxia attenuated the $\mathrm{aDP}$. Interestingly, high concentrations of $A \beta$ did not have this effect. Concurrent application of $A \beta$ and a Panx 1 antibody was not additive on the effect of $A \beta$ or the antibody alone. Lastly, $\mathrm{A} \beta$ did not block excitotoxic currents during NMDAR overstimulation.

Conclusion: These data suggest that $A \beta$ acts as a neuroprotective agent during hypoxia/ischemia by interacting with Panxl. This gives insight into the physiological activity of $A \beta$ and can provide better understanding of the underlying cause of stroke-induced AD.

\section{Novel stress rescues homotypic stress-induced cannabinoid receptor downregulation}

Laura Senst ${ }^{1,2,3}$

Jaclyn I. Wamsteeker Cusulin ${ }^{1,2}$

Jaideep S. Bains ${ }^{1,2}$

${ }^{1}$ Hotchkiss Brain Institute,

${ }^{2}$ Department of Physiology and Pharmacology

3 Cumming School of Medicine, University of Calgary, Calgary, $\mathrm{AB}$ 
Background: The paraventricular nucleus lies at the apex of the hypothalamic-pituitary-adrenal (HPA) axis stress response, and displays bi-directional plasticity. Synapses here respond to retrograde messengers including the ubiquitous endocannabinoids (eCBs). Both $\mathrm{eCB}$ and cannabinoid type-1 receptor $(\mathrm{CB} 1 \mathrm{R})$ signalling modulates the output of the HPA axis in response to stress. Our lab has previously demonstrated that, in adolescent male rats, repetitive exposure to homotypic stress functionally downregulates CB1R signalling at synapses on parvocellular neuroendocrine cells (PNCs) in the paraventricular nucleus. Following stress exposure, CB1R function passively recovers over several days. Here, we hypothesized that recovery of CB1R signalling would be sensitive to synaptic and behavioural manipulations.

Methods: Experiments were performed using male Sprague Dawley rats (post-natal day 21-35). The stress paradigm used was a mixed psychological and physical stressor, 30-min immobilization. In the novel stress condition a different stress paradigm (forced swim or predator odor) was substituted on the last day. Coronal hypothalamic brain slices were prepared for in-vitro whole-cell patch clamp recordings of PNCs. Depolarization-induced suppression of inhibition (DSI) at GABAergic synapses was used to identify retrograde short-term plasticity and activation level of CB1R.

Results and conclusions: GABA synapses in PNCs of naive animals showed robust depolarization- induced suppression of inhibition $(45.2 \pm 4.9 \%$ inhibition of post-synaptic currents, $\mathrm{n}=28, \mathrm{p}<0.01)$, which was eliminated following 5-day immobilization stress $(10.6 \pm 2.5 \%, \mathrm{n}=26, \mathrm{p}<0.01)$. Interestingly, exposing an animal to a novel forced swim stress on the fifth day caused a robust recovery of CB1R signalling (63.1 $\pm 9.3 \%, n=7, p<0.01)$. eCB signalling completely recovered with low frequency stimulation $(1 \mathrm{~Hz}, 10 \mathrm{~min})$ of PNCs following 5D stress, suggesting that novel stress may modify CB1 signalling through alterations to presynaptic activity state. These observations indicate that $\mathrm{eCB}$ signalling is labile and can be regulated in a bi-directional manner by homotypic and novel stressors.

\section{Altered functional connectivity at sites of malformation of cortical development}

CA Beers ${ }^{1,2}$

A Cull ${ }^{1}$

I Gaxiola-Valdez ${ }^{1}$

Daniel J Pittman ${ }^{2}$

Paolo Federico ${ }^{1,2}$

${ }^{1}$ University of Calgary

${ }^{2}$ Hotchkiss Brain Institute, Calgary, AB

Rationale: Over the past decade, resting state functional magnetic resonance imaging (rsfMRI) has emerged as a method for characterizing network phenomena in the brain. Areas with correlated signal changes over time are deemed to be connected with one another. RsfMRI studies of epileptic patients have demonstrated alterations in typical brain connectivity within various cognitive domains including language, motor and memory function, as well as widespread network abnormalities. For example, patients with mesial temporal lobe epilepsy have decreased connectivity within the epileptogenic temporal lobe and increased connectivity to extra-temporal sites. Malformations of cortical development (MCD) are a common cause of epilepsy and can be associated with both focal and widespread network alterations. In this study, we examined rsfMRI connectivity changes associated with MCDs in patients with epilepsy compared with healthy controls.

Methods: Seven subjects diagnosed with epilepsy and MCDs, confirmed by structural MRI, were recruited: two subjects with focal nodular heterotopia; two with polymicrogyria; and three with focal cortical dysplasia. Each subject underwent a five-minute rsfMRI scan while looking at a projected fixation cross using a 3 T GE scanner (GE, Waukesha, WI). Regions of interest (ROIs) were generated encompassing the entire MCD for each subject. These ROIs were used to assess the connectivity between the MCD and the rest of the brain. These data were then compared with five age-matched healthy controls, looking for areas of increased or decreased connectivity at the sites of malformation.

Results: In 6/7 (85\%) subjects, the MCDs showed significantly less global connectivity when compared with analogous healthy tissue in the control subjects. Also, the MCDs had greater connectivity within the malformation ROI compared with the analogous site in healthy controls. One subject demonstrated significantly greater global connectivity at the MCD compared with healthy controls. The type of malformation did not impact the connectivity findings. 
Conclusions: Based on these data, MCDs showed less connectivity to the rest of the brain compared with analogous healthy tissue. In contrast, there was increased connectivity within the MCD itself compared with healthy tissue. These data suggest that focal MCDs may cause widespread network disturbances that result in relative isolation of the malformed tissue.

\section{Analysis of left ventricular filling dynamics}

Lindsay Burrowes ${ }^{1}$

Alessandro Satriano ${ }^{2}$

Richard Thompson ${ }^{3}$

Nigel Shrive ${ }^{1}$

John Tyberg ${ }^{2}$

1 Schulich School of Engineering, University of Calgary, Calgary, $\mathrm{AB}$

2 Libin Cardiovascular Institute, University of Calgary, Calgary, $\mathrm{AB}$

3 Department of Biomedical Engineering, University of Alberta, Edmonton, $\mathrm{AB}$

Introduction: During diastole, the left ventricle (LV) fills with blood in two phases: late filling; and early filling. Late filling, resulting in the mitral flow "A-wave", is a result of left atrial contraction. Early filling, manifest as the "E-wave", is thought to be substantially due to diastolic suction (DS), a phenomenon where the LV aspirates blood and fills itself, independent of atrial activity. To study DS, we have invasively measured pressure and used cardiac MRI to evaluate cavitary volume and flow (velocity). Here we demonstrate the ability to quantify changes in DS resulting from varied experimental conditions.

Methods:

Experimental: The study was performed in pigs $(\mathrm{n}=15)$ and was approved by the Animal Care Committee. Pressure was measured in the LV and aorta (high-fidelity) and in the right atrium, as an estimate of pericardial pressure $(n=5)$. Cardiac MRI captured a full 3-chamber short-axis (SAX) stack, and 2-, 3- and 4-chamber long-axis (LAX) views. Phase-contrast MRI was performed for a 3-chamber SAX at the level of the mitral valve and in mutually orthogonal directions in the 3-chamber LAX view.

Computational: Pressure and MRI data could not be recorded simultaneously from their respective measuring devices, so were temporally aligned by making end diastole (i.e., $0.1 \mathrm{x}$ maximum $\mathrm{dP}_{\mathrm{LV}} / \mathrm{dt}$ ) simultaneous with Phase 1 of the image sequence. Alignment was refined by relating maximal and minimal LV volumes to fiducial pressure events. Only after optimal alignment was further analysis performed. All analysis was performed in Matlab (The MathWorks, Natick, MA). Pressure gradients were calculated using Euler's equation along a user-determined line drawn from the top of the left atrium to the LV apex. Wave intensity analysis (WIA), which uses independent measures of pressure and velocity to determine the direction and nature of waves, was calculated from the LV pressure and measured mitral velocity. The energy of the early diastolic backwards decompression wave (BDW; a pressure-decreasing and flow-increasing wave) was calculated. LV volumes were determined using GIUSEPPE, an in-house program which incorporates the full SAX stack as well as the 2-, 3- and 4-chamber LAX views. Pressure-volume (PV) loops were then plotted to study the amount measure filling prior to minimum LV pressure $\left(\mathrm{PLV}_{\min }\right)$.

Results: The amount of filling occurring before $P L V_{\min }$ was compared with the energy contained in the BDW after the opening of the mitral valve. The analyzed results indicate that the amount of filling that occurs before $P L V_{\text {min }}$ may be related to ESV but that the energy of the BDW may not be related to ESV. The benefit of WIA is its unique ability to discriminate an atrial "push" from a ventricular "pull". Pressure gradients, as derived solely from mitral inflow velocity, do not appear to provide any additional information beyond velocity studies alone.

Conclusions: Preliminary analysis indicates that DS can be quantified by both PV loop analysis and WIA however further analysis will continue in order to study if there is a relationship between these measurements. 
Cardiovascular magnetic resonance imaging of cardiac remodelling and strain analysis in adolescent obesity

\author{
Anna Schmidt ${ }^{1}$ \\ Alessandro Satriano ${ }^{1}$ \\ Kate Fenwick ${ }^{1}$ \\ Madeline Arnold ${ }^{1}$ \\ Andrew Howarth ${ }^{1}$ \\ Daniele Pacaud ${ }^{2}$ \\ Bobby Heydari 1,3 \\ James White ${ }^{1,3}$ \\ Matthias Friedrich ${ }^{4}$
}

1 Stephenson Cardiovascular Imaging Center, Libin Cardiovascular Institute of Alberta, Calgary, AB

2 Division of Paediatric Endocrinology, Department of Paediatrics, University of Calgary, Calgary, $\mathrm{AB}$

${ }^{3}$ Division of Cardiology, Department of Medicine, University of Calgary, Calgary, AB

${ }^{4}$ Division of Cardiology, Department of Medicine, McGill University, Montreal, QC

Background: The worldwide increased prevalence of childhood obesity has resulted in a rise in cardiometabolic disease including type 2 diabetes and cardiovascular disease (CVD) within an obese population. Cardiovascular magnetic resonance (CMR) imaging provides a sensitive imaging tool that may afford early detection of structural and functional alterations secondary to obesity. This pilot study explores the potential for CMR imaging, inclusive of left ventricular (LV) volume and mass analysis, native T1 mapping and 3D strain analysis to identify such changes related to obesity in adolescence.

Methods: Eleven subjects between the ages of 10 and 20 years were prospectively recruited from a Pediatric Weight Clinic, and defined as obese if they were $\geq 95^{\text {th }}$ percentile of weight for their age. Fourteen age and gender-matched healthy weight control subjects were also enrolled. All subjects had baseline anthropometric and laboratory measures in addition to a standardized CMR imaging protocol, inclusive of routine multi-planar cine imaging and native T1 mapping using the shMOLLI pulse sequence. Left ventricular (LV) volumetric analysis and T1 mapping were blindly analyzed using cvi 42 (Circle Cardiovascular, Calgary, AB) and 4D strain analysis was performed using GIUSEPPE in-house software.

Results: The mean age of the obese group was $14 \pm 1$ years versus $14 \pm 2$ years in the control group $(p=0.572)$. BMI was higher in the obesity cohort $\left(32 \pm 6\right.$ and $21 \pm 4 \mathrm{~kg} / \mathrm{m}^{2}$, $\mathrm{p}<0.0001)$ as was systolic blood pressure (SBP) serum lipid levels and markers of insulin resistance. The LV ejection fraction was non-significantly elevated in the obese group $(62.8$ $\pm 4.7 \%$ vs $59.1 \pm 5.4 \%, \mathrm{p}=0.084)$. A trend was seen for elevation in LV mass index $\left(26.1 \pm 5.9\right.$ vs $21.6 \pm 6.7 \mathrm{~g} / \mathrm{m}^{2.7}$, $\mathrm{p}=0.084$ ) with the mean segmental wall thickness significantly elevated in the obese cohort $(5.5 \pm 0.8$ vs $4.6 \pm 0.8 \mathrm{~mm}$, $\mathrm{p}=0.019)$. BMI was positively correlated with LV mass index $(\mathrm{r}=0.648, \mathrm{p}<0.0001)$, wall thickness $(\mathrm{r}=0.708, \mathrm{p}<0.0001)$ and mass:volume ratio $(\mathrm{r}=0.415, \mathrm{p}=0.039)$. The $4 \mathrm{D}$ strain analysis revealed significant elevations in peak circumferential endocardial strain $(-20.5 \pm 2.1$ vs $-18.7 \pm 2.0 \% \mathrm{p}=0.04)$ and peak longitudinal endocardial strain $(-16.7 \pm 1.6$ vs $-14.4 \pm 1.9 \%$ $\mathrm{p}=0.006)$ in the obese cohort, consistent with a hyper-contractile state. Native T1 mapping was not significantly different between the groups $(954 \pm 21$ vs $954 \pm 31$ $\mathrm{ms}, \mathrm{p}=0.985$ ).

Conclusion: In this pilot study we showed childhood obesity to be associated with objective elevations in LV mass, LV wall thickness and mass:volume ratio. In this early stage of remodelling, these structural changes were not associated with elevation in native T1, a surrogate marker of tissue fibrosis. Strain analysis revealed a hyper-contractile state, likely representing a combined contribution of elevated systolic blood pressure with compensatory myocyte hypertrophy. Longitudinal study into the effects of childhood obesity on LV remodelling with multi-component CMR protocols are warranted.

\section{Mapping the full extent of the seizure onset zone using high frequency oscillations recorded simultaneously with functional MRI: A case report}

Aaron M Spring

Craig A Beers

Anita T Kang

Ismael Gaxiola-Valdez

Daniel J Pittman

Paolo Federico

University of Calgary, Calgary, AB

Introduction: Pathological high frequency oscillations (HFOs) recorded via intracranial electroencephalography (iEEG) have recently emerged as a marker for the seizure onset zone (SOZ) in patients with focal epilepsy, and have been 
shown to be more specific to the SOZ than conventional markers such as interictal discharges (IEDs). Like IEDs, the detection of HFOs is limited to the tissue surrounding implanted electrodes. We aim to overcome this limitation by capitalizing on the spatial resolution of functional magnetic resonance imaging (fMRI) through the use of simultaneous iEEG-fMRI to map the spatial extent of the HFO-associated network, and correlate the post-resection outcome with the overlap between that network and the area of resection in a patient with post-traumatic epilepsy and left temporal lobe seizures.

Methods: Data (11 minutes of iEEG data) were collected at 20 $\mathrm{kHz}$ simultaneously with $\mathrm{fMRI}$ at $3 \mathrm{~T}$. The data were artifact rejected, decimated to $2 \mathrm{kHz}$ and filtered for HFOs (80-250 $\mathrm{Hz}$ ). HFO events were detected using in- house developed software and IED events were visually identified by an experienced epileptologist. HFO and IED event timings were used to generate $\mathrm{MMRI}$ activation maps of the respective event types. The active fMRI clusters were assessed to determine their relationship to the area of resection using an anatomical MR scan acquired following surgical resection of the presumed seizure focus.

Results: Events (310 HFO events) were detected within the 11-minute scan. The HFO-associated fMRI activity was comprised primarily of two clusters: one completely contained within the resection margins of the temporal lobe; and the other located in the ipsilateral parietal lobe. In comparison, none of the IED-associated networks overlapped with the area of resection. The patient achieved a positive surgical outcome with no recurrence of dyscognitive or convulsive seizures as of the 2-year follow-up.

Discussion: We have overcome several technical limitations in order to acquire the high quality recordings necessary to perform the aforementioned analyses, which will be implemented in a larger cohort of patients to formally investigate the findings of this case study. These data support existing evidence of the superior specificity of HFOs to the SOZ compared with conventional markers. While preliminary, these data are the first to establish the feasibility of analyzing iEEG data collected simultaneously with fMRI for HFOs, and may provide a promising new tool for localizing the SOZ.

\section{Sensory-motor network connectivity in children with perinatal stroke}

KE Woodward ${ }^{1}$

H Carlson ${ }^{1}$

E Zewdie ${ }^{1}$

A Kuczynski

J Saunders

J Hodge

A Kirton ${ }^{1,2}$

${ }^{1}$ Department of Neuroscience

${ }^{2}$ Calgary Pediatric Stroke Program, University of Calgary

Background: Perinatal stroke causes most hemiparetic cerebral palsy and lifelong disability. Human developmental plasticity models are defining sensorimotor neurophysiology; identifying central targets for neuromodulation. Network connectivity is poorly understood but can be defined with resting-state fMRI (rsfMRI). We hypothesized that sensorimotor connectivity is measureable with rsfMRI in perinatal stroke and associated with functional outcome.

Methods: Children were recruited through the Alberta Perinatal Stroke Project (APSP), a population-based cohort. Subjects aged 6-18 years with MRI-defined periventricular venous infarction (PVI) were compared with controls. Resting-state BOLD signal was acquired on 3T MRI with children at rest for 5 minutes. Data was analyzed using the FEAT tool of FSL. Functional connectivity was computed between four anatomical nodes: S1 and M1 of the left/stroke and right/non-stroke hemispheres; and a control node (right temporal pole). Primary outcome was connection strength (coefficient of determination, $r^{2}$ ). Subjects were ranked for motor outcome (Assisting Hand Assessment, AHA), proprioceptive dysfunction (robotic position matching variability, VAR) and corticospinal arrangement defined by single pulse TMS.

Results: Twenty-five subjects included 10 PVI (mean age $13.50+/-3.85$ ) and 15 control subjects (age 12.67+/-3.89 years). The network was measureable in all subjects. Strong, symmetrical INTRAhemispheric M1-S1 correlations observed bilaterally in controls (left $=0.782+/-0.10$, right $=0.785+/-0.11)$ were comparable in PVI (stroke $=0.758+/-0.11$, non-stroke $=0.776+/-0.09)$. INTERhemispheric correlations were weaker and comparable between cases and controls: $\mathrm{M} 1-\mathrm{M} 1=0.371,0.366$, $S 1-S 1=0.368,0.398$. Correlations to right temporal pole were all $<0.1$. Functional outcomes AHA and VAR were not associated with network connectivity. Subjects with 
prominent ipsilateral CST arrangements had the weakest INTRAhemispheric M1-S1connectivity, consistent with PVI sensory-motor dissociation theory.

Conclusions: Sensorimotor network connectivity in perinatal stroke can be assessed with rsfMRI. INTRAhemispheric M1-S1 connectivity is robust across normal and stroke children but may be disordered in some patterns of reorganization. Future connectivity analyses need to assess larger samples, wider networks and interventional effects.

\section{Development of high-sensitivity torque magnetometers using cavity optomechanics}

\author{
L Nathanael \\ $\mathrm{Y} \mathrm{Wu}^{1,2}$ \\ Marcelo $\mathrm{Wu}^{1,2}$ \\ Tayyaba Firdous ${ }^{2,3}$ \\ Fatemeh F. Sani ${ }^{2,3}$ \\ Joseph E. Losby 2,3 \\ Mark R. Freeman ${ }^{2,3}$ \\ Paul E. Barclay ${ }^{1,2}$
}

${ }^{1}$ Institute for Quantum Science and Technology, University of Calgary, Calgary, AB

${ }^{2}$ National Institute for Nanotechnology, Edmonton, $\mathrm{AB}$

3 Department of Physics, University of Alberta, Edmonton, $\mathrm{AB}$

Introduction: Ultralow-field magnetometers are powerful magnetic field sensors that can be used in a variety of applications, ranging from the study of magnetic materials, geology, memory storage, defense, space exploration and medicine. Recently, there has been a push towards developing highly sensitive miniaturized devices with operational ranges extended into ambient conditions. Such developments could pave the way to significant advances in various fields including magnetic resonance imaging by allowing increased image resolution and operation at lower magnetic field strengths, thus significantly reducing the current costs and imaging constraints associated with using a superconducting magnet to generate the required magnetic fields.

Methods: One approach to developing highly sensitive miniaturized magnetometers is to apply recent advances from photonics and the burgeoning field of cavity optomechanics. To that end, we developed a nanoscale torque magnetometer involving the formation of a nanocavity from two collinear nanophotonic beams. Incorporating magnetic material onto one of the beams allowed the beam to be mechanically driven by external magnetic fields. These mechanical deformations were then sensitively detected by light trapped within the nanocavity, thus allowing the device to behave as a very sensitive magnetic fieldsensor.

Results: The devices were designed and then produced from silicon-on-insulator substrates using electron beam lithography. A record-breaking magnetic moment sensitivity of $10^{9}(\mathrm{~A} / \mathrm{m})$ was measured for these devices in ambient conditions. The devices were sensitive enough to detect nanoscale defects within the magnetic material.

Conclusion: By applying design principles from cavity optomechanics, highly sensitive torque magnetometers were developed and found to have record-breaking magnetic moment sensitivities in ambient conditions.

\section{Dose-dense paclitaxel with carboplatin vs intraperitoneal cisplatin with paclitaxel for the treatment of advanced ovarian cancer}

Sarah Glaze, MD, FRCSC ${ }^{1}$

Amanda Forsyth, $\mathrm{MSc}^{2}$

${ }^{1}$ Department of Gynecologic Oncology, Tom Baker Cancer Centre, Calgary, AB

2 Cumming School of Medicine, University of Calgary, Calgary, $\mathrm{AB}$

Introduction: Ovarian cancer is the leading cause of death from gynecological cancers in Canada. It is estimated that 2,800 Canadian women will be diagnosed with ovarian cancer in 2015, and that 1,750 women will die from it. The current standard treatment in most centres consists of a combination of cytoreductive surgery and platinum-based chemotherapy. The order of treatment is determined by stage at presentation; women with more advanced stage at presentation are treated with chemotherapy first. Standard adjuvant chemotherapy after surgery consists of combined intravenous carboplatin and paclitaxel given every three weeks, but intravenous paclitaxel and intraperitoneal cisplatin (IV/IP) in three-week intervals is also used in many centres in North America after this protocol was shown to have considerable benefit in 2006; however, the use of the IP catheter can be logistically challenging with worsening side effectsfor patients.

In 2009, a large Japanese trial showed a survival benefit for patients given intravenous paclitaxel with carboplatin in a dose-dense weekly protocol. The Tom Baker Cancer Centre in 
Calgary was the first centre in North America to adopt a dose-dense protocol for treatment of advanced ovarian cancer in 2010. The objective of this study is to determine if dose-dense chemotherapy has provided similar patient outcomes as IV/IP chemotherapy in our patient population.

Methods: Data from all patients treated with dose-dense paclitaxel with carboplatin or cisplatin with paclitaxel at the Tom Baker Cancer Centre, Calgary, AB between 2006 and 2014 is currently being retrospectively extracted from medical records. Demographic information, patient characteristics, treatment type, adverse events, disease recurrence and patient survival are being recorded.

Results: Data collection is currently ongoing; however, a previous review of 46 patients undergoing treatment with dose-dense paclitaxel and carboplatin since March 2010 showed the Calgary protocol to be a feasible option in our population. Sixty-one percent of women completed the protocol with minimal interruption, and the most commonly reported grade 3 and 4 toxicities were fatigue, neuropathy and neutropenia. In comparison to the landmark Japanese dose-dense study, fewer women experienced adverse events, particularly hypomagnesemia and neutropenia, which is likely due to our regular administration of magnesium and prophylactic filgrastim.

Conclusion: Dose-dense paclitaxel with carboplatin is a practical treatment option for advanced ovarian cancer. We anticipate that this study will support the feasibility of the Calgary dose-dense chemotherapy protocol and provide a comparison with IV/IP chemotherapy to determine if it provides comparable, if not better, outcomes for our patient population.

\section{Magnetic resonance imaging in awake preschool-age children: An evaluation of different training protocols}

Ashleigh Frayne ${ }^{1}$

Deborah Dewey ${ }^{2,3}$

Catherine Lebel 2,3

${ }^{1}$ Department of Radiology

2 Department of Pediatrics

${ }^{3}$ Community Health Sciences, University of Calgary, Calgary, $\mathrm{AB}$

Introduction: Magnetic resonance imaging (MRI) is a non-invasive technique useful for numerous research and clinical applications; however, this technique is sensitive to motion and often requires a considerable amount of time for image acquisition, making it difficult to implement in young pediatric populations. In a clinical setting, sedation or general anesthesia is often used to overcome these difficulties, but these are not appropriate in a research setting. Previous studies have utilized a variety of strategies and preparation procedures to mitigate the practical challenges of scanning young children. These strategies include scanning children during natural sleep, training children in a mock scanner and using play therapy. This study aimed to identify and assess the efficacy of training methods for preschool age children undergoing multiple MRI scans.

Methods: Children $(n=122$, mean age 3.3 years, range 2.3-4.7 years) were initially recruited from the Calgary community and the Alberta Pregnancy Outcomes and Nutrition (APrON) Study. Different preparation techniques were used to prepare children for MRI scanning, including training in a mock scanner or immersion in a space-adventure story. Imaging was performed at the Alberta Children's Hospital on the research-dedicated 3T GE MR750w MRI scanner with a 32-channel head coil. Chi- squared tests were used to assess probability of success in children receiving mock scanner training or story training. Total length of time spent in the scanner and the total number of sequences successfully completed were compared between groups using independent samples t-tests.

Results: Of the 122 children initially recruited, 89 (73\%) successfully completed at least one scan. Of these 89 children, 86 (97\%) completed two or more scans. The majority of children $(n=82,67 \%)$ were trained with the space-adventure story only. There were no significant differences in demographics between groups trained with different preparation techniques. Children trained with the mock scanner were significantly less likely to be successful than those trained without $(p=0.004,40 \%$ success with: $78 \%$ success without). The space-adventure story was not a significant predictor of success, but demonstrated a trend relationship ( $\mathrm{p}=0.087,76 \%$ success with: $63 \%$ success without) toward increasing success in children who participated. Among those with successful scans, participants trained using the space-adventure story spent a statistically significantly longer time on average $(41 \mathrm{~min} \pm 11 \mathrm{~min})$ in the scanner than other 
children ( $34 \mathrm{~min} \pm 10 \mathrm{~min}, \mathrm{p}=0.005)$ and were able to successfully complete more sequences (six versus five sequences, $\mathrm{p}=0.013)$. Use of the mock scanner was not significantly associated with number of sequences completed or total minutes in the scanner.

Conclusion: Overall, 89 preschool-age children successfully completed at least one MRI scan during this study, demonstrating the feasibility of conducting MRI exams in awake preschool-age children, without use of sedatives or general anesthesia. Significant differences between children trained with or without the mock scanner may suggest a selection bias, as parents were offered a choice to complete training in the mock scanner, and most parents who thought their children would be successful opted out of the extra visit. Our finding that children participating in the space-adventure story spent significantly longer in the scanner compared with other children suggests that stories and props increase compliance for MRI scanning in young children.
Results from the EVVA study: Prevalence of anal intraepithelial neoplasia and acceptability of screening for anal cancer among women living with HIV

Elaina Kaufman ${ }^{1}$

Alexandra de Pokomandy ${ }^{1,2}$

Christina de Castro ${ }^{2}$

Marie Munoz $1,2,3$

Bernard Lessard $1,2,4$

Marie-Hélène Mayrand 5

Louise Charest ${ }^{3}$

Manon Auger ${ }^{6}$

Victoria Marcus ${ }^{6}$

Ann Burchell ${ }^{7}$

Marina Klein ${ }^{2}$

François Coutlée $e^{2,8}$

1 Department of Family Medicine, McGill University, Montreal, QC,

2 Chronic Viral Illness Service, McGill University Health Centre, Montreal QC

${ }^{3}$ Clinique Médicale L'Actuel, Montréal QC

${ }^{4}$ Clinique Médicale du Quartier Latin, Montréal QC

5 Department of Obst. \& Gyn., Centre Hospitalier de l'Université de Montréal, Montréal QC

6 Pathology Department, McGill University Health Centre, Montreal QC

${ }^{7}$ Ontario HIV Treatment Network, Toronto, ON

${ }^{8}$ Department of Microbiology \& Infectious Diseases, Centre Hospitalier de l'Université de Montréal, Montréal, QC

Background: Given the disproportionately high rates of anal cancer in people living with HIV, routine anal cancer screening has been recommended for this population. Various tools are being considered for screening, but they must be acceptable to the individuals being screened in order for screening programs to be successful. While anal cancer screening acceptability has been studied in men who have sex with men (MSM), this acceptability is not known for women living with HIV (WLHIV).

This sub-study measures the prevalence of anal intraepithelial neoplasia (precancerous lesions known as AIN) and assesses the acceptability of screening for anal cancer in WLHIV, within the EVVA study ("Evaluation of HPV, HIV and AIN in women").

Methods: EVVA is an ongoing cohort study of 150 WLHIV in Montreal, Canada, involving biannual cervical/anal human papillomavirus testing and cytology over two years. A 
systematic high-resolution anoscopy (HRA) and digital ano-rectal examination (DRE) were performed at baseline and at two years. An acceptability questionnaire was completed at two years or the subject was withdrawn from the study.

Results: Of 150 women enrolled, 75 had completed the acceptability questionnaire at time of analysis. Prevalent high-grade AIN was identified in 17\% (24/141; 95\% confidence interval [CI]:11-23) at baseline. Among questionnaire respondents, $73 \%$ (55/75; 95\% CI:63-84) considered routine anal cancer screening in WLHIV an absolute necessity. Pain during anal swabs and DRE was similar to cervical swabs (median grade: $1 / 10$ ), while HRA was considered more painful than cervical swabs (median grade: $6 / 10)$. Yearly cervical swabs were considered very acceptable by $80 \%$ (60/75; 95\%CI:71-89) of respondents. Anal swabs were considered very acceptable yearly by $75 \%$ (56/75; 95\%CI:65-85), every two years by 93\% (70/75; $95 \% \mathrm{CI}: 88-99)$, and every 5 years by $95 \%$ (71/75; 95\%CI:89-100). DRE was considered very acceptable yearly by 79\% (59/75; 95\%CI:69-88), every 2 years by $92 \%$ (69/75; $95 \% \mathrm{CI}: 86-98)$, and every five years by $93 \%$ (70/75; 95\%CI:88-99). HRA was considered very acceptable every two years by $77 \%$ (58/75; $95 \%$ CI:68-87), every five years by $91 \%$ (68/75; 95\%CI:84-97), and every 10 years by $93 \%$ (70/75; 95\%CI: 88-99). Pain was a key reason for low acceptability. Embarrassment, feelings of trauma, inconvenience and perceived non-necessity were also reported in written comments.

Conclusion: AIN is highly prevalent in WLHIV. The vast majority of participants to date considered screening necessary and very acceptable. Pain management can be improved and potential adverse psychological effects of screening should be explored.

\section{Pilot study of an interdisciplinary educational and support program for patients with neovascular age-related macular degeneration (AMD)}

Shehzad Kassam ${ }^{1}$

Carissa Kratchmer ${ }^{2,3}$

Abid Valji ${ }^{1}$

Imran Jivraj ${ }^{1,2}$

Matthew Tennant ${ }^{1,2}$

${ }^{1}$ University of Alberta, Edmonton, $\mathrm{AB}$

${ }^{2}$ Alberta Retina Consultants, Edmonton, $\mathrm{AB}$

${ }^{3}$ University of Calgary, Calgary, AB

Background: Age-related macular degeneration (AMD) is a progressive disease of the retina and is the leading cause of chronic vision loss in older adults. Several studies indicate that $20 \%-30 \%$ of patients with the disease report depressive symptoms. There is a need for interdisciplinary programs to educate and support patients living with AMD.

Methods: Six didactic modules were developed with each covering different topics related to AMD: ocular exam and anatomy, AMD biology, treatment, healthy living, vision rehabilitation and mood. Patients diagnosed with neovascular AMD within one year prior to enrollment were randomly assigned to one of three interventions: didactic lectures; a combination of didactic lectures with a support group component; or standard clinical care. Participants were assessed using10-item Likert scale measuring knowledge about AMD, stress levels, hopefulness, perceived support and empowerment at baseline and six weeks post-randomization. Patient satisfaction was determined using qualitative surveys.

Results: Forty-six participants participated in the study with an average age of $78.2 \pm 7.3$ years; $52.1 \%$ were male. Patients had a diagnosis of AMD for $5.7 \pm 3.5$ months. Knowledge levels rose by $23.0 \%$ in the control group, $41.3 \%$ in the didactic group and $35.6 \%$ in the support group. Stress levels rose by $16.3 \%$ in the control group but decreased in the didactic $(-11.9 \%)$ and support $(-27.5 \%)$ groups. Hopefulness declined in the control group $(-7.9 \%)$, remained stable in the support group $(0.1 \%)$ and increased in the didactic (10.8\%) group. Levels of perceived support remained stable in the control $(-1.9 \%)$ and support $(-2.6 \%)$ groups but rose in the didactic group (12.9\%). Levels of empowerment declined in the control group (-11.3\%), and the didactic group (-6.8\%), but slightly increased in the support group (3.0\%). Overall, patients were highly satisfied with the content presented in the six modules using qualitative measures. 
Conclusion: The study did not have the power to show any significant differences in outcome following the didactic and combined interventions compared with standard care or preand post-intervention; however, the data are suggestive of an increase in knowledge, hopefulness and perceived support, as well as a reduction in stress levels following education and support sessions. It is also worth noting that patient satisfaction with the program was high overall. Future study should aim for a larger sample size and follow up period. More research is required to determine the interventions effectiveness in addressing depressive symptoms and improving patient's quality of life.

\section{The effect of temperature on metered-dose inhalers}

Chelsea M.D. Morin

James W. Ivey

Jordan T.F. Titosky

Jonathan D. Suderman

Jason S. Olfert

Reinhard Vehring

Warren H. Finlay

Department of Mechanical Engineering, University of Alberta, Edmonton, $\mathrm{AB}$.

Introduction: Inhaler testing is typically conducted at standardized atmospheric conditions: at room temperature with a constant pressure and humidity. Patients do not always use their inhalers in a controlled environment, leading to an unknown dose of medication actually reaching the small airways of their lungs. The purpose of this study is to look at various temperature conditions at which patients may use their inhalers and compare the in vitro lung dose at room temperature with the in vitro lung dose at various other temperature conditions.

Materials and methods: Four metered-dose inhalers (MDIs) were used in this study: Symbicort ${ }^{\circ}$, Ventolin ${ }^{\circ}$, Airomir ${ }^{\mathrm{TM}}$ and QVARTM. Inhalers were actuated into the Alberta Idealized Throat, a leading model for testing inhaler devices, and the samples were collected on filters and weighed. Testing conditions included equilibrated inhaler and environmental temperatures of approximately $-10^{\circ} \mathrm{C}, 0^{\circ} \mathrm{C}, 10^{\circ} \mathrm{C}, 20^{\circ} \mathrm{C}$ and $40^{\circ} \mathrm{C}$ as well as room temperature inhalers at approximately $20^{\circ} \mathrm{C}$ actuated in environmental temperatures of approximately $0^{\circ} \mathrm{C}$ and $-10^{\circ} \mathrm{C}$. The Alberta Idealized Throat was kept at approximately $37^{\circ} \mathrm{C}$ and the humidity was kept below $1 \%$ relative humidity.

Results: A significant decrease in the in vitro lung dose for equilibrated inhaler and environmental conditions was seen for all four devices when comparing temperatures of approximately $-10^{\circ} \mathrm{C}$ and $20^{\circ} \mathrm{C}(\mathrm{p}<0.01)$. At $-10^{\circ} \mathrm{C}$, in vitro lung deposition for Symbicort, Ventolin, Airomir and QVAR was $11 \%(\mathrm{SD}=3 \%), 19 \%(\mathrm{SD}=7 \%), 28 \%(\mathrm{SD}=4 \%)$ and $56 \%$ $(\mathrm{SD}=14 \%)$, respectively, of that at $20^{\circ} \mathrm{C}$. When the devices were kept at room temperature and actuated in ambient temperatures of approximately $0^{\circ} \mathrm{C}$ and $-10^{\circ} \mathrm{C}$, both Ventolin and Airomir showed significant reduction in the in vitro lung dose $(\mathrm{p}<0.05)$.

Conclusions: Temperature has a significant impact on the performance of metered-dose inhalers. Each inhaler was impacted differently, suggesting that formulation or thermally-induced changes to the valve, seal or other inhaler components may contribute to the in vitro lung dose. Evaporation rates are slower in lower ambient temperatures, suggesting that the volatile components may not have time to completely evaporate, increasing the momentum of the particles and thus increasing oropharyngeal deposition. This study suggests that it is important to ensure that patients are mindful of using their devices in conditions as close to ideal as possible, to ensure that the optimal dose is delivered to their lungs.

\section{An investigation of parent and child sleep patterns on an in-patient oncology unit}

Gaya Narendran ${ }^{1,2}$

Soeun Lee ${ }^{1}$

Lianne Tomfohr 3,4

Fiona Schulte $1,2,4$

1 Cumming School of Medicine, University of Calgary, Calgary $\mathrm{AB}$

2 Oncology, Hematology and Transplant, Alberta Children's Hospital, Calgary, AB

3 Alberta Children's Hospital, Calgary, AB

${ }^{4}$ Department of Psychology, University of Calgary, Calgary $\mathrm{AB}$

Introduction: Sleep is vital for innumerable processes in a developing child. Pediatric cancer patients and their caregivers often stay in hospital for extended periods of time and report 
disruptions in usual sleep patterns. The primary aim of this research is to study objective as well as subjective measures of sleep quality in pediatric cancer patients, as well as their parents, receiving care in the oncology ward at the Alberta Children's Hospital.

Methods: The effects of sleep disruption were investigated as they relate to the fatigue and level of anxiety experienced by this population. Data obtained from this research will help to identify ways to decrease the effects of an important physiological consequence of current cancer care practices thus, results from this study will be used to develop guidelines for hospital practices (i.e., the frequency of routine blood draws) relevant to sleep interruption in order to enhance the quality of the experience of staying in the hospital.

Results: Hospitalized pediatric cancer patients experienced significantly disrupted sleep experiences, compared with previously published data outlining the sleep experience of pediatric cancer patients who sleep in their home environment. Increased nocturnal awakenings translated into greater self-reported anxiety and mood disturbance the following day, in both patients and their parents.

Conclusions: Importantly, our study focuses on the total family unit and aimed to address both the physical and psychological aspects of wellness in children and their families who are impacted by pediatric cancer. This study is still ongoing but our preliminary findings indicate that $80 \%$ of pediatric oncology patients self- reported symptoms of sleep disruption and/or fatigue based upon measures of Child Sleep Habits, Anxiety and Sleep Quality. Additionally, the PedsQL Multifatigue Scale indicated that all eight children reported greater general, cognitive and sleep/rest fatigue in comparison with a control group of 163 children hospitalized for renal function observation.

\section{Highlighting the perspectives of nephrologists on sex hormone status in women with chronic kidney disease: A survey of Canadian nephrologists}

Sharanya Ramesh ${ }^{1}$

Ellen Wells Seely ${ }^{2}$

Matthew T. James 1,2

Jayna M. Holroy-Leduc ${ }^{3}$

Stephen B. Wilton ${ }^{3,4}$

Sofia B. Ahmed ${ }^{1,3,4}$

1 Department of Medicine, Cumming School of Medicine, University of Calgary, Calgary, $\mathrm{AB}$

2 Brigham and Women's Hospital, Boston, MA

3 Community of Health Sciences, University of Calgary, Calgary, $\mathrm{AB}$

${ }^{4}$ Libin Cardiovascular Institute of Alberta, University of Calgary, Calgary, AB

Background: Chronic kidney disease (CKD) in women is often accompanied by menstrual and fertility disorders due to kidney-mediated endocrine disturbances. Whether this issue is recognized by nephrologists and discussed by them with their patients is unknown.

Methods: We electronically surveyed 124 nephrologists in Canada, identified via Canadian Society of Nephrology membership ( $44 \%$ response rate), regarding their impressions of sex hormone status, menstrual cycle, fertility and the role of postmenopausal hormone therapy (HT) in women with CKD. Responses were stratified by kidney transplant nephrologist status.

Results: Twelve percent of respondents were transplant nephrologists. There were no differences in age or sex between transplant and non-transplant nephrologists. Ninety-three percent of respondents agreed that kidney function has an important impact on regulation of sex hormones; however, only $36 \%$ reported discussing fertility, and even fewer (13\%) reported discussing menstrual irregularities with their patients. Only 39\% of respondents advised their patients to address these concerns with their family physician or an endocrinologist or gynecologist. Most of the nephrologists responded that they do not know if there is a role for postmenopausal HT in patients with CKD (47\%) and 22\% of nephrologists disagreed with the statement.

Nephrologists did not know whether the potential benefits of postmenopausal HT outweighed the risks in CKD $(50 \%)$ or if the formulation (39\%), route of administration 
(41\%), and time of HT initiation (50\%) play a role in the actions of postmenopausal hormone therapy.

Conclusion: While nephrologists recognized the impact of CKD on sex hormone status in women, few addressed fertility and menstrual disorders with their patients. Our survey highlights existing uncertainties of nephrologists about how to manage disturbances of sex hormones in people with CKD.

\section{Sex doesn't matter: Capillary blood volume and diffusing capacity response to exercise in men and women}

Melissa Bouwsema ${ }^{1,2}$

Vince Tedjasaputra ${ }^{1}$

Michael K. Stickland ${ }^{2}$

${ }^{1}$ Faculty of Physical Education and Recreation

2 Faculty of Medicine and Dentistry, University of Alberta, Edmonton, $\mathrm{AB}$

Introduction: Previous work suggests that women exhibit a greater respiratory limitation to exercise compared with height-matched men. Pulmonary capillaries are recruited with incremental exercise to increase pulmonary diffusing capacity. Because of their smaller lungs, women may potentially have a reduced pulmonary capillary blood volume $\left(\mathrm{Vc}_{\mathrm{c}}\right)$ and membrane diffusing capacity $(\mathrm{Dm})$ available to meet the increased oxygen demand. We hypothesized that females would have a lower $\mathrm{Vc}$ at rest and during exercise compared with males.

Methods: Sixteen females $(112 \pm 12 \%$ predicted relative $\left.\mathrm{VO}_{2 \max }\right)$, and sixteen males $(118 \pm 22 \%$ predicted relative $\left.\mathrm{VO}_{2 \max }\right)$ were matched for height and weight. $\mathrm{Hb}$ corrected diffusing capacity (DLCO), Vc, and Dm were determined via the multiple-FIO2 DLCO technique at rest, and during incremental exercise up to $90 \%$ of $\mathrm{VO}_{2 \max }$.

Results: Both groups demonstrated an increase in DLCO, Vc, and $\mathrm{Dm}$ with exercise intensity, but women had $20 \%$ lower DLCO $(p<0.001), 18 \%$ lower Vc $(p=0.002)$, and $22 \%$ lower $\mathrm{Dm}(p<0.001)$ compared with men across all workloads. When these variables were corrected for alveolar volume (VA), the between-sex difference was eliminated.

Conclusion: Women demonstrate consistently lower DLCO, Vc and Dm compared with height-matched men; however, these differences disappear with corrected for lung size, indicating that any difference between men and women can be explained by lung size, and not an intrinsic sex difference in the pulmonary vasculature.

Supported by: Natural Sciences and Engineering Sciences Council of Canada and Alberta Innovates Health Solutions

\section{Progressive resistance exercise for head and neck cancer patients during and following radiation treatment: results from the randomized, controlled ENHANCE trial}

Lauren C Capozzi ${ }^{1,2}$

Harold $\mathrm{Lau}^{3,4}$

Margaret L McNeely5

Raylene A Reimer ${ }^{1,6}$

Janine Giese-Davis 3,7

S Nicole Culos-Reed ${ }^{1,3,7}$

${ }^{1}$ Faculty of Kinesiology, University of Calgary, Calgary, AB

2 Cumming School of Medicine, University of Calgary, Calgary, $\mathrm{AB}$

${ }^{3}$ Department of Oncology, Faculty of Medicine, University of Calgary, Calgary, $\mathrm{AB}$

4 Department of Oncology, Tom Baker Cancer Centre, Alberta Health Services, Calgary, AB

${ }^{5}$ Department of Physical Therapy, University of Alberta \& Cross Cancer Institute, Edmonton, AB

6 Department of Biochemistry \& Molecular Biology, Cumming School of Medicine, University of Calgary, Calgary, $\mathrm{AB}$

7 Department of Psychosocial Resources, Tom Baker Cancer Centre, Alberta Health Services, Calgary, AB

Introduction: Head and neck cancer (HNC) and related treatments affect a multitude of basic physiological and psychological functions, leading to decreased nutritional status, impaired physical functioning and decreased quality of life (QOL). There is a growing body of literature to support the use of physical activity (PA) in managing these side effects; however, it is unclear what the role of progressive resistance exercise and lifestyle education is for HNC patients, and the optimal timing of intervention initiation (before radiation initiation or post-radiation completion).

Methods: Sixty HNC patients were recruited to participate in a lifestyle intervention trial evaluating the primary outcome of body composition (lean body mass, body mass index and body fat), as well as secondary outcomes of fitness, QOL and nutrition status. Patients were randomized to a 12-week 
progressive resistance training and lifestyle intervention either during radiation treatment (immediate lifestyle intervention from baseline to 12-week assessment, ILI) or immediately following completion (delayed lifestyle intervention from 12-week to 24-week assessment, DLI). Assessments occurred before the start of radiation and at 12, 24, 36 and 48-weeks post diagnosis. The first three assessment time points (up to 24 weeks) are discussed here.

Results: Despite group differences in PA across the 24-weeks, the ILI and DLI groups did not differ significantly for lean body mass $(F(2,74.5)=0.280, p=0.756)$, BMI $(\mathrm{F}(2,74.9)=0.361, \mathrm{p}=0.698)$ or percent body fat $(\mathrm{F}(2,75.9)=0.301, \mathrm{p}=0.741)$. Follow-up analysis revealed a significant decline in all body composition scores by 12 -weeks, which remained at 24-weeks, regardless of group assignment. While statistically significant between-group differences were not detected across the first 12-week period for body composition, Cohen's $d$ effect size calculations revealed a small effect of the ILI on lean body mass ( $d=0.130), B M I(d=0.183)$, and percent body fat $(\mathrm{d}=0.146)$ when compared with the DLI group. A small to medium intervention effect was also noted for secondary outcomes, with improved fitness scores (sit and reach $\mathrm{d}=0.407$, total grip strength $\mathrm{d}=0.517)$, QOL $(\mathrm{d}=-0.207)$, and nutrition status $(\mathrm{d}=-0.289)$.

Conclusions: HNC patients experienced a similar decline in body composition during and following radiation treatment, regardless of lifestyle intervention timing. Despite this, effect size calculations supported intervention benefits, suggesting larger multi-site trials investigating the impact of lifestyle intervention on HNC patients following their cancer diagnosis are warranted.

\section{The See Kidney Disease (SeeKD) targeted screening program for chronic kidney disease}

Lauren Galbraith, BSc ${ }^{1}$

Paul Ronksley, $\mathrm{PhD}^{1}$

Lianne Barnieh, $\mathrm{PhD}^{2}$

Joanne Kappel, MD MSc${ }^{3}$

Braden Manns, MD, MSc1,2

Susan Samuel, MD, MSc ${ }^{2}$

Min Jun, $\mathrm{PhD}^{2}$

Rob Weaver, $\mathrm{MSc}^{2}$

Nadine Valk ${ }^{4}$

Brenda Hemmelgarn, MD, $\mathrm{PhD}^{1,2}$

${ }^{1}$ Department of Community Health Sciences, University of Calgary, Calgary, $\mathrm{AB}$

${ }^{2}$ Department of Medicine, University of Calgary, Calgary, AB

3 Division of Nephrology, University of Saskatchewan, Saskatoon, SK

${ }^{4}$ Kidney Foundation of Canada, Ottawa, ON

Background: The effectiveness of targeted screening for identification of chronic kidney disease (CKD) is largely unknown. The See Kidney Disease (SeeKD) targeted screening project aimed to determine the prevalence of unrecognized CKD in Canada.

Study Design: Cross-sectional.

Setting and Participants: The SeeKD project was conducted across Canada with events to identify adults with risk factors for CKD (e.g., diabetes, hypertension, vascular disease and family history of kidney problems).

Outcomes and measurements: Participants with at least one risk factor for CKD received a point-of-care creatinine measurement to identify unrecognized CKD (estimated glomerular filtration rate $[\mathrm{eGFR}]<60 \mathrm{~mL} / \mathrm{min} / 1.73 \mathrm{~m}^{2}$ ). Baseline information collected included clinical characteristics, sociodemographics and health knowledge. Semi-structured telephone interviews were conducted with each branch after the screening events to characterize local screening strategies, which were subsequently categorized as individual-targeted (specifically targeting individuals at risk of CKD) and community-targeted (event in a community location in proximity to a high risk population). We calculated the prevalence of unrecognized CKD overall, and by screening strategy.

Results: Between January 2011 and February 2014, 6,329 Canadians participated in the SeeKD screening events. Participants were predominantly female (65.3\%), middle-aged 
(mean $=58.5$ years) and the majority $(88.9 \%)$ self-reported at least one risk factor for CKD. Of participants with at least one risk factor, 92.3\% $(\mathrm{N}=5,194)$ were screened for $\mathrm{CKD}$, of whom $18.8 \%$ (95\% confidence interval [CI] 17.8-19.9) had unrecognized CKD; the majority (13.8\%) had stage 3a CKD (eGFR $45-60 \mathrm{~mL} / \mathrm{min} / 1.73 \mathrm{~m}^{2}$ ). The prevalence of unrecognized $\mathrm{CKD}$ was higher for branches with individualversus community-targeted events (21.9\% (95\% CI 20.5-23.4) vs. $14.7 \%$ (95\% 13.2-16.2)).

Limitations: CKD was defined using a single creatinine measurement.

Conclusions: Targeted screening identified a high proportion of individuals with risk factors for CKD and a high prevalence of unrecognized CKD. Future research will evaluate the ability of targeted screening to promote self-management behaviours addressing priorities for people with CKD.

\section{Sulcoflex piggyback intraocular lens implantation for correction of refractive errors following cataract surgery}

Mona Purba, BSc OD ${ }^{1}$

Cynthia Mardinger, $\mathrm{BHSc}^{2}$

Abby Hibma, $\mathrm{BSc}^{3}$

Howard V. Gimbel, MD MPH FRCSC1,4

${ }^{1}$ Gimbel Eye Centre, Calgary, AB

${ }^{2}$ Faculty of Medicine, University of Calgary, Calgary, $\mathrm{AB}$

3 Faculty of Medicine, Loma Linda University, Loma Linda, CA USA

${ }^{4}$ Department of Surgery, University of Calgary, Calgary, AB

Introduction: Refractive errors following cataract surgery can affect visual outcomes and decrease patient satisfaction. The purpose of this study was to investigate the visual and refractive outcomes following implantation of a Sulcoflex intraocular lens (IOL) to correct pseudophakic refractive errors.

Methods: This retrospective chart review included 14 pseudophakic eyes of 13 patients. All eyes underwent implantation of a secondary piggyback IOL (Sulcoflex Rayner $653 \mathrm{~L}$ or $653 \mathrm{~T}$ ) placed in the ciliary sulcus. The mean patient age at treatment was 64 years. Visual outcomes were determined based on target refraction, postoperative refraction and uncorrected distance visual acuity (UDVA) measurements.
Results: Eleven eyes $(78.6 \%)$ had a postoperative spherical equivalent (SE) within 0.50 diopters (D) of the targeted SE. Preoperative UDVA was $20 / 30$ in four eyes (28.6\%), and $20 / 40$ or worse in 10 eyes $(71.4 \%)$. Postoperative UDVA was $20 / 30$ or better in all eyes, where half $(50.0 \%)$ of eyes were $20 / 20$ or better. The mean preoperative $\log$ MAR $(0.50 \pm$ 0.33 ) significantly improved to $0.06 \pm 0.09, p<.01$. There were no complications.

Conclusions: The significant improvement in UDVA and the precision in reaching the target refraction suggest that the Sulcoflex lens is a viable and successful treatment option for pseudophakic residual refractive error.

\section{Insulin receptor signalling is necessary for the maintenance of epithelial phenotype in MCF10A cells}

Vanessa Di Palma ${ }^{1,2}$

Vuk Stambolic ${ }^{1}$

1 Department of Medical Biophysics, Princess Margaret Cancer Center, University of Toronto, Toronto, ON

2 Undergraduate Medicine University of Calgary, Calgary, AB

Introduction: Obesity is an adverse factor in the development and severity of breast cancer (BC). Obesity is often accompanied by an increase in circulating insulin, which is associated with a poor BC prognosis. Compared with women of normal weight, overweight women with $\mathrm{BC}$ have a higher risk of death and distant recurrence. The obesity-related increase in circulating insulin levels is accompanied by a decrease in insulin sensitivity in insulin-target tissues. High fasting insulin levels are associated with a poor prognosis of $\mathrm{BC}$, and insulin resistance syndrome triples $\mathrm{BC}$ recurrence. Finally, the type 2 diabetes drug metformin, which effectively lowers circulating insulin levels, reduces risk and severity of cancer in diabetics.

Although not expressed in normal breast tissue, the insulin receptor (IR) is highly expressed in $\mathrm{BC}$ tissue, raising the possibility that insulin signalling in BC cells may be responsible for the negative prognostic effects associated with hyperinsulinemia. Considering that insulin has essential metabolic functions, modelling the relationship among obesity, high insulin levels and their impact on $\mathrm{BC}$ represents a major challenge.

Methods: This project describes the development of a cell-based system (the non-transformed breast epithelial cell line, MCF10A) to study the mechanisms by which insulin affects BC. Through both depletion of insulin from their 
growth media, as well as lentiviral knockdown of the IR in these cells in $2 \mathrm{D}$ and $3 \mathrm{D}$ growth, changes in morphology, migration, growth and signalling in response to insulin were characterized. By inhibition of downstream signalling in the insulin receptor pathway, including AMPK which is inhibited by the DM2 drug Metformin, the level at which signalling through the IR induced the observed changes was determined.

Results: MCF10A human breast epithelial cells, which express the IR and are untransformed, require insulin signalling for normal proliferation and morphology. Interestingly, hyperactivation of ERK1/2 in MCF10A cells was seen in response to insulin withdrawal, resulting in a loss of epithelial phenotype. Unexpectedly, while losing epithelial phenotype, MCF10A cells depleted of insulin failed to migrate.

Conclusion: Breast cells that expressed IR required insulin for migration and maintenance of epithelial characteristics.

\section{Inhibition of colonic apithelial apoptosis by Proteinase-Activated Receptor 2 occurs independently of MMP activation and EGFR transactivation}

\author{
Vadim Iablokov \\ Elizabeth Trusevych \\ Mike Dicay \\ Wallace K. MacNaughton
}

Inflammation Research Group, Department of Physiology and Pharmacology, Snyder Institute for Chronic Diseases, University of Calgary, Calgary, $\mathrm{AB}$

Introduction: Mucosal biopsies from patients with inflammatory bowel disease (IBD) display significantly increased levels of epithelial apoptosis compared with biopsies from healthy individuals. IBD likely arises due to an inappropriate immune response to intestinal microbiota, which develop in genetically susceptible individuals with a defective epithelial barrier. Understanding the pathways that regulate intestinal epithelial homeostasis may provide additional targets for IBD therapy. A potential candidate for maintaining intestinal epithelial homeostasis is the $G$ protein-coupled receptor, proteinase-activated receptor 2 (PAR2), which is expressed on epithelial cells. We previously showed that PAR2 inhibits IFN- $\gamma$ - and TNF- $\alpha$-induced apoptotic cell death in colonic epithelial cells through MEK1/2- and PI3K-dependent pathways. Here we sought to identify how PAR2 activates these pro-survival pathways in order to block apoptosis in colonic epithelial cells.
Methods: HT-29 colonic epithelial cells expressing PAR2 were pretreated with small-molecule or antibody antagonists of epidermal growth factor (EGFR; AG-1478, PD153035 and Cetuximab) as well as matrix metalloproteinases (MMPs; Marimastat, GM6001) in serum-free media. The levels of phosphorylated ERK1/2 and Akt in response to the PAR2 agonist 2-furoyl-LIGRLO (2f-LI) were then determined by Western blotting. The same inhibitors as above were then used to determine whether PAR2 stimulates MMP and EGFR activity to decrease apoptosis. HT-29 cells were stimulated with $2 \mathrm{fLI}$ for one hour and apoptosis was induced with 40 $\mathrm{ng} / \mathrm{ml} \mathrm{IFN-} \gamma$ and $10 \mathrm{ng} / \mathrm{ml}$ TNF- $\alpha$ for six hours. Levels of cleaved poly (ADP-ribose) polymerase (PARP) and cleaved caspase-3 were measured by Western blotting as markers of apoptosis.

Results: MMP inhibition with Marimastat or GM6001 significantly blocked ERK1/2 phosphorylation stimulated by 5 $\mu \mathrm{M} 2 \mathrm{fLI}$ in a dose-dependent manner. Both inhibitors partially but significantly decreased PAR2-induced Akt phosphorylation. Blocking EGFR with AG-1478 or PD153035 significantly decreased the phosphorylation of ERK1/2 in response to $2 \mathrm{fLI}$. The level of Akt phosphorylation remained significantly elevated above baseline with the highest concentration of EGFR inhibitors. Pre-treating HT-29 cells with Cetuximab, an EGFR-specific antibody that blocks ligand binding, significantly reduced phosphorylation of both ERK1/2 and Akt; however, inhibition of MMPs and EGFR with any of the compounds tested did not block the ability of $2 \mathrm{fLI}$ to reduce apoptosis stimulated by IFN- $\gamma$ and TNF- $\alpha$.

Conclusions: Activation of PAR2 stimulated ERK1/2 phosphorylation dependent on the activity of MMPs and EGFR. Phosphorylation of Akt was not dependent on MMPs but was dependent on ligand binding to EGFR. In spite of this, PAR2 inhibited apoptosis by a mechanism not involving MMPs nor EGFR. Further work is required to elucidate the mechanism by which PAR2 facilitates epithelial cell survival. 
The majority of children with epilepsy are affected by postictal phenomena, often preventing a return to normal activities of childhood

Sarah J. MacEachern ${ }^{1,2}$

Nancy Thornton ${ }^{2}$

Jeffrey R. Buchhalter ${ }^{1,2}$

1 Cumming School of Medicine, University of Calgary, Calgary $\mathrm{AB}$

2 Alberta Children's Hospital Research Institute, Alberta Children's Hospital, Calgary AB

Rationale: Following a seizure, patients with epilepsy have reported diverse symptoms in the postictal period, from headache to psychosis, with varying degrees of frequency, duration and severity. These phenomena are not well characterized, and their impact on patient well-being is not understood. The aim of this study is to characterize postictal symptoms in a population of children with epilepsy.

Methods: We proposed that, in a subset of epilepsy patients, postictal symptoms will affect their ability to return to normal childhood behavior. To test this hypothesis, we used a questionnaire-based approach to characterize postictal symptoms, including type, frequency and duration in a population of children with epilepsy, and we evaluated the impact these symptoms had on the ability of these children to perform their regular activities. Additionally, we sought to identify aggravating and alleviating factors. Ethics approval was obtained from the Conjoint Health Research Ethics Board (CHREB) at the University of Calgary.

Results: Preliminary data was analyzed from 154 patients seen in the outpatient Neurology Clinic at the Alberta Children's Hospital (average age: 9 [0-17]; 48\% F, 52\% M). Initial results found that $88 \%$ of respondents experienced postictal symptoms $(135 / 154)$, with the most common symptom being fatigue (90\%; 121/133; Fig 1) and the most bothersome being weakness, which prevented $79 \%$ of those affected $(n=55 / 70)$ from returning to normal activities of childhood. These data will be correlated with age, gender, ethnicity, seizure type and epilepsy syndrome.

Conclusions: With this preliminary study, we hope to further our understanding of symptoms experienced in the postictal period and gain a better understanding of how these symptoms impact children with epilepsy. To the best of our knowledge, this is the first prospective study of this type in the pediatric population.

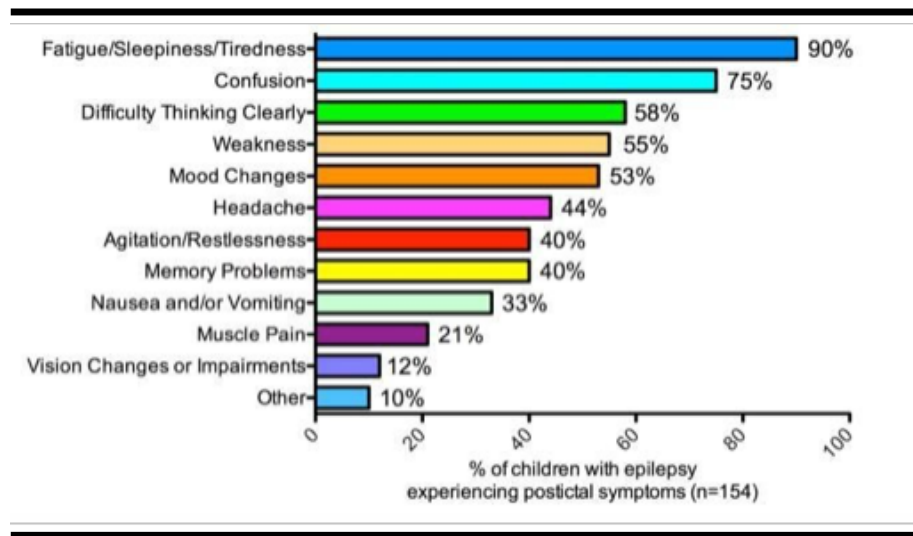

FIGURE 1. Percentage of children with epilepsy experiencing specific post-ictal symptoms. $\mathrm{n}=154$

\section{Introduction of a pilot nicotine awareness program for school age youth in Pincher Creek, AB}

Meghan Olson

Jacqueline Williamson

Gavin Parker, MD

Tobias Gelber, MD

Cumming School of Medicine, University of Calgary, Calgary $\mathrm{AB}$

Introduction: Over the past 15 years, the market for novel nicotine products, such as smokeless tobacco and electronic cigarettes, has expanded greatly. Many of these products are targeted towards youth and are marketed as safer alternatives to traditional cigarettes. Despite the popularity of these products, there are very few resources available to raise awareness about the potential health risks they may carry. The goal of this project was to develop a presentation for school aged youth to increase awareness about the risks of various nicotine products.

Methods: The presentation, titled "Up in Smoke: Trends, Truths and Tall Tales" focused on the risks associated with cigarettes, smokeless tobacco, and electronic cigarettes. Two medical students delivered the 30 - minute presentation to 79 students in grades 5-10 in Pincher Creek Alberta. Students were given short Likert Scale surveys to complete before and after the presentation. Time was dedicated afterwards for questions and student-led discussion.

Results: In the initial survey data, $>75 \%$ of participants knew someone who used nicotine products, yet $<30 \%$ knew how to find accurate information about potential risks associated with 
their use. Before the presentation, electronic cigarettes and smokeless tobacco were perceived to pose less health risk than traditional cigarettes. After the presentation all three products had similar risk perception.

Conclusion: The presentation was extremely well received by students and teachers, and was an effective way to raise awareness about the risks associated with novel nicotine products.

Future directions: The program will be continued in the 2015-2016 school year in most the schools in the Pincher Creek area. Given the interest expressed by the schools, we hope to expand the program to include an interactive component, and create two slightly different visual presentations geared towards different age ranges.

\section{Health related behaviors and comorbidity in people with epilepsy: changes in the past decade}

Jodie I. Roberts ${ }^{1,2,3}$

Scott B. Patten 2,3,4

Samuel Wiebe ${ }^{1,2,3}$

Brenda R Hemmelgarn 3,5

Tamara Pringsheim $1,2,3,4,6$

Nathalie Jetté1,2,3

1 Department of Clinical Neurosciences, University of Calgary, Calgary, AB

${ }^{2}$ Hotchkiss Brain Institute, University of Calgary, Calgary, AB

3 Department of Community Health Sciences and O'Brien Institute for Public Health, University of Calgary, Calgary, AB ${ }^{4}$ Department of Psychiatry and Mathison Institute for Mental Health Research \& Education, University of Calgary, Calgary, $\mathrm{AB}$

${ }^{5}$ Department of Medicine, University of Calgary, Calgary, AB

${ }^{6}$ Department of Pediatrics, University of Calgary, Calgary, AB

Introduction: Epilepsy is associated with negative health-related behaviors (HRBs), including smoking and poor participation in physical activity compared with the general population. However, there are reasons to be optimistic that HRBs in people with epilepsy (PWE) have improved. Recommendations regarding physical activity for PWE have changed and many benefits of exercise for PWE have been demonstrated. Additionally, the prevalence of cigarette smoking among the adult general population is declining and consequently similar declines may have occurred in PWE. It is unknown whether these HRBs have improved over time, and if potential changes in HRBs have impacted the prevalence of comorbidities. We aimed to explore estimates of HRBs and comorbidities in PWE and examine whether behaviors have changed over the past decade.

Methods: A repeated cross-sectional study was conducted using data from a series of five cycles (2001-2011) of the Canadian Community Health Survey, a national population-based survey. The proportions and odds ratios with 95\% confidence intervals for HRBs and comorbidities in PWE compared with respondents without epilepsy were calculated for each survey over the 10-year period and estimates were examined for changes over time.

Results: The study included 522,722 participants of which $3,220(0.6 \%)$ had epilepsy. The proportion of PWE who did not participate in physical activity decreased over time (2001=17.2\%, 2010/2011=8.5\%), as did the proportion of PWE who smoked cigarettes $(2001=32.3 \%$, 2010/2011=18.0\%). PWE consistently reduced alcohol consumption in comparison to the general population. Less than half of participants met the recommended daily guidelines for fruit and vegetable consumption. An apparent reduction in the prevalence of heart disease occurred in PWE $(2001=11.7 \%, 2010 / 2011=4.0 \%)$, but not in those without epilepsy; however, similar trends were not observed for other cardiovascular comorbiditiesstudied.

Conclusion: Apparent improvements in smoking cessation and physical activity occurred in PWE. Despite these improvements, physicians should continue to counsel their patients on the importance of healthy lifestyle choices. Further exploration into the reasons for the apparent decrease in the prevalence of heart disease in PWE is warranted. 


\section{Accuracy of venous blood gas sodium and potassium results compared to standard serum laboratory measurements}

Omar F. Khan, $\mathrm{MD}^{1}$

Barbora Sporinova, $\mathrm{BSc}^{2}$

Christopher Naugler, MSc, MD 3

Aleem M. Bharwani, MD

${ }^{1}$ Internal Medicine Residency Program, Cumming School of Medicine, University of Calgary, Calgary, AB

2 Undergraduate Medical Education Program, Cumming School of Medicine, University of Calgary, Calgary, AB

3 Department of Pathology and Laboratory Medicine, Cumming School of Medicine, University of Calgary, Calgary, $\mathrm{AB}$

${ }^{4}$ Department of Medicine, Cumming School of Medicine, University of Calgary, Calgary, AB

Introduction: Sodium and potassium disturbances are frequently encountered across all patient populations. Electrolyte disturbances increase length of stay, hospital costs, morbidity and mortality, and thus an accurate and rapid assessment of electrolytes is needed to prevent medical complications and decrease healthcare costs. Currently, serum electrolyte measurement can take over $60 \mathrm{~min}$ before results are reported. Measuring electrolytes using venous blood gases (VBGs) can yield results within minutes; however, the data regarding accuracy of VBG electrolytes is sparse and equivocal. This study aimed to assess the accuracy of VBG electrolyte values in comparison with serum electrolyte values in a generalized patient population.

Methods: Data from all patients presenting to four Calgary Emergency Departments between December 1-31, 2014, and who had concurrently drawn VBGs and serum electrolyte panels, were retrospectively extracted from medical records. Factors analyzed included patient age, gender, disposition from the Emergency Department, collection time and Emergency Department discharge diagnosis for all sets of blood draws.

Results: Data were collected on 1,005 concurrent VBG and electrolyte panel blood draws from patients presenting to Emergency, of which 1003 were used for potassium analysis and 1,004 for sodium analysis. Strong correlations were observed between VBG and serum electrolyte panel potassium (Spearman Rank Correlation, $\mathrm{R}=0.912)$ and sodium $(\mathrm{R}=$ $0.940)$ levels. Sodium levels were overestimated slightly by VBGs (mean Nadiff value of $+1.45 \mathrm{mmol} / \mathrm{L}$ ) compared with serum electrolytes, while potassium levels were not (mean
Kdiff value of $0.0045 \mathrm{mmol} / \mathrm{L}$ ). Overall, 960 blood draws (95.7\%) had VBG potassium levels within $0.5 \mathrm{mmol} / \mathrm{L}$ of the serum electrolyte panel level, while 953 (94.9\%) had VBG sodium levels within $4 \mathrm{mmol} / \mathrm{L}$ of the electrolyte panel (these values are within currently accepted limits). Negative predictive values were found to be especially notable with electrolyte imbalances when using VBGs. VBGs were highly specific for sodium and potassium disorders, however their sensitivity was variable. There were no clinically significant differences in results among various patient demographics or disease factors.

Conclusion: Our study demonstrated that venous blood gas electrolyte sodium and potassium values have excellent correlation with serum electrolyte panel levels, as well as strong negative predictive values for sodium and potassium disorders.

\section{Examining the psychometric properties of three standardized screening tools in a pregnant and parenting population}

Ingunn Benediktsson ${ }^{1}$

Sheila McDonald 2,3

Suzanne Tough ${ }^{1,2,4}$

${ }^{1}$ Faculty of Medicine, University of Calgary, Calgary, AB

${ }^{2}$ Faculty of Medicine, Department of Pediatrics, University of Calgary, Calgary, $\mathrm{AB}$

3 Alberta Health Services, Maternal and Child Health, Research and Innovation, Public, Population and Aboriginal Health

${ }^{4}$ Department of Community Health Sciences, Faculty of Medicine, University of Calgary, Calgary, AB

Introduction: Although standardized tools such as the Speilberger State Anxiety scale (1970) and Cohen's Perceived Stress Index (1983) have often been used in pregnant and postpartum populations to evaluate psychosocial risk, there exists very little literature on the reliability of these scales in this population. Given the potentially grave consequences of undiagnosed pre- and postpartum mental distress, it is of paramount importance that the tools that are commonly used are evaluated for their psychometric properties within the pregnant and parenting population.

Methods: The psychometric properties of three mental health scales were evaluated using the All Our Babies data. The All Our Babies cohort is a prospective longitudinal study based in Calgary, Alberta. A total of 3,300 women were recruited from 
prenatal care sites from all four quadrants of the city. Participants were mailed questionnaires at 24-26 weeks gestation, 34-36 weeks and at four months postpartum. Reliability for the Speilberger State Anxiety Scale, the "Perceived Stress Index and the Life Optimism Test Revised" were evaluated by calculating Cronbach's alphas.

Validity with related constructs was tested for the State Anxiety Scale and the Perceived Stress Index by calculating Pearson Correlation Coefficients with closely related constructs.

Results: The Cronbach's Alphas for the Speilberger State Anxiety scale were 0.92, 0.92 and 0.93 for 24-26 weeks, 34- 36 weeks and four months postpartum respectively. The alpha's for the Perceived Stress index were 0.88, 0.88 and 0.89, respectively. The Life Optimism Test Revised was only measured in the third trimester and the Cronbach's alpha was 0.83. The Pearson Correlation Coefficient for Anxiety with depression were $r=0.73, r=0.72$ and $r=0.77$ respectively. The coefficients for stress and depression were $r=0.75, r=0.75$ and $\mathrm{r}=0.77$ respectively.

Discussion and conclusion: The psychometric properties for all three scales were strong, with alphas that were comparable to or higher than literature values. These data provide evidence that the use of these scales, previously validated in other populations, are appropriate for use in among pregnant and parenting women at risk for mental distress.

\section{Drug abuse and misuse: A report on prescription-monitoring program underuse}

Jennifer Chen

Sonja Wicklum, MD

Cumming School of Medicine, University of Calgary, Calgary, $\mathrm{AB}$

Introduction: Did you know that Canada is the second largest consumer of prescription opioids in the world? Did you also know that there has been a significant increase in the number of people seeking treatment for addiction to prescription drugs? In the first two months of 2015 alone, there were 50 deaths in Alberta connected to fentanyl abuse. Drug abuse and misuse is an undisputable and fatal epidemic that must be urgently addressed.

In 2013, the National Advisory Council on Prescription Drug Misuse published "First Do No Harm: Responding to Canada's Prescription Drug Crisis", in which it strongly endorses Prescription-Monitoring Programs (PMP) as a strategy in combatting prescription drug abuse. Alberta has been a frontrunner in leading this effort - the College of Physicians \& Surgeons of Alberta (CPSA) has been collecting prescribing and dispensing data for listed drugs in Alberta since 1986. However, the data collected under the Triplicate Prescription Program is under- utilized, and the PMP itself continues to be undermined by problems of user accessibility, prescription forgery, theft, and loss, as well as questionable prescribing behaviours. This study aimed to explore the evidence behind PMPs, evaluate Alberta's current PMP, summarize PMPs of other provinces/territories, and make recommendations for achievable change.

Methods: A literature search was performed with Medline, Pubmed, Google as well as on the websites of CPSA, Alberta College of Pharmacists, and Canadian Centre on Substance Abuse in June 2015. Keywords searched were "prescription monitoring program", "substance related disorder", "opioid related disorder", "drug and narcotic control", "narcotics", "doctor shopping", "multiple prescribers". This study and the authors' recommendations are built upon the findings of the "2012 Alberta Triplicate Prescription Program Atlas" and the "2015 Prescription Monitoring Programs in Canada: Best Practice and Program Review".

Results: The evidence behind PMPs comes from observational studies, case studies, expert opinions and accumulated experience or perception. Benefits of PMPs include lower rates of doctor shopping and changed prescribing behaviour; however, studies show that healthcare providers are often not accessing information available through PMPs.

Alberta's PMP has several ideal characteristics, but its shortcomings result in data under- utilization by healthcare providers. In order to optimize PMP outcomes and effectively reduce drug abuse and misuse, the authors have made specific recommendations for immediate and long-term changes to Alberta's PMP. A national concerted effort will be necessary to improve patient care and standardize practice across Canada. 
Monitoring quality of care for joint replacements: Assessing alternative statistical methods to accurately estimate time to revision

Sarah Lacny ${ }^{1}$

Peter D Faris ${ }^{2,3}$

Eric Bohm ${ }^{4}$

Linda J Woodhouse ${ }^{5,6,7}$

Otto Robertsson ${ }^{8}$

Deborah A Marshall, $\mathrm{PhD}^{1,3,7}$

1 Department of Community Health Sciences, Faculty of Medicine, University of Calgary

2 Alberta Health Services

${ }^{3}$ Alberta Bone and Joint Health Institute

${ }^{4}$ Department of Surgery, Section of Orthopaedic Surgery, University of Manitoba

5 Alberta Health Services Bone and Joint Health Strategic Clinical Network

${ }^{6}$ Department of Physical Therapy, University of Alberta

${ }^{7}$ McCaig Institute for Bone and Joint Health

8 The Swedish Knee Arthroplasty Register, Department of Clinical Sciences, Orthopedics, Lund University

Background: With increased longevity and frequency with which joint replacements are being performed on younger, more physically active patients, it has become common for patients to outlive the life of their prosthesis and require a revision. The cumulative incidence of revision (i.e., revision rate) provides a measure of the failure rate of joint replacements and can be used to project future demand for revisions.

Due to varying patient follow-up times and censoring, survival analysis is required to estimate revision rates. The most commonly applied method, the Kaplan-Meier (KM) method, does not account for the competing risk of death and consequently overestimates revision rates. Our objective was to assess alternative methods for estimating revision rates using hip and knee replacement data from Alberta, Canada, over a 10 year period and knee replacements recorded in the Swedish Knee Arthroplasty Register (SKAR) over 24 years. We recommend a preferred method that can be used to inform health policy decisions.

Methods: Time to revision, death, or censoring were measured for cohorts of total hip arthroplasties $(n=12,496)$ and total knee arthroplasties (TKAs) $(\mathrm{n}=19,172)$ in Alberta from 2003 to 2013 and TKAs $(n=80,177)$ in Sweden from 1989 to 2012. Cumulative incidence of revision were estimated using the KM failure function and the cumulative incidence function (CIF), which accounts for competing risks. Relative difference (RD) were calculated between KM and CIF estimates. Three regression models were compared: the Cox proportional hazards model; Fine and Gray subdistribution hazards model; and Royston and Parmar flexible parametric model. We adjusted for age, sex, Charlson Comorbidity Score (Alberta cohorts only) and year of the primary operation (Swedish cohort only). Our base case analysis included primary unilateral operations only. In sensitivity analysis, we included unilateral and staged-bilateral operations.

Results: The KM cumulative incidence estimate was greater than the CIF at each time point, and the magnitude of overestimation increased with follow-up time. The magnitude of overestimation was greatest for the Swedish knee cohort, which also had the highest cumulative incidence of death due to its longer follow-up time and older population. At five years, the RDs between the cumulative incidence estimated using the KM method and the CIF for the Alberta hip, Alberta knee and Swedish knee cohorts were $1.8 \%, 2.3 \%$, and 3.8\%, respectively. At nine years, the RDs increased to $3.1 \%, 5.8 \%$, and $8.2 \%$, respectively. At 23 years, the $\mathrm{RD}$ for the Swedish cohort reached $39.1 \%$, where the cumulative incidence estimated using the $\mathrm{KM}$ method was $7.4 \%$ compared with $5.3 \%$ for the CIF. Each adjusted regression model yielded similar coefficients, standard errors and P-values for our base case analysis. In sensitivity analysis, the Fine and Gray subdistribution hazard ratio differed from the hazard ratios obtained from the Cox and Royston and Parmar models ( $\mathrm{P}=0.365, \mathrm{P}=0.039, \mathrm{P}=0.045$, respectively).

Conclusions: Our results support the application of the CIF and competing risks regression models to more accurately estimate the cumulative incidence of revision following joint replacement surgery for the purposes of healthcare planning and resource allocation. 


\section{Jump power reflects the degree of disability in} neuromuscular disease

\section{Christopher Newell ${ }^{1}$}

Barbara Ramage ${ }^{2}$

Alberto Nettel-Aguirre 3,4

Ion Robu², Aneal Khan ${ }^{3,5}$

${ }^{1}$ Department of Medical Science

${ }^{2}$ Neurosciences

3 Pediatrics

${ }^{4}$ Community Health Sciences

${ }^{5}$ Medical Genetics, Faculty of Medicine, University of Calgary and Alberta Children's Hospital,

Calgary, $\mathrm{AB}$

Rationale: Mitochondrial diseases can impair muscle function and restrict mobility. Motor disability can affect the ability to work, ambulate and quality of life. Clinical measures of functional ability currently exist (timed chair up-and-go, 6-minute walk test, grip strength, etc.) although tests that apply functional measures in patients with mitochondrial disease are scarce. Whether to measure degenerative changes in mobility or response to therapy, a reproducible and easy-to-perform functional measure is needed. We therefore developed a novel application of peak jumping power (PJP) as a quantitative index of functional mobility in mitochondrial disease patients.

Objectives: Determine if PJP can measure the functional ability in patients with mitochondrial disease.

Methods: Adult and pediatric patients were recruited from the metabolic diseases clinic and healthy control subjects through a recruitment poster. Subjects performed five successive jumps from a squat position, without countermovement, while standing on a force plate. Mass and instantaneous force were used to compute velocity from which instantaneous power (watts) and the best result was divided by the mass $(\mathrm{kg}$ ) to give the PJP (watts $/ \mathrm{kg}$ ). Ambulatory ability was recorded as healthy controls (HC), patients with no reported functional weakness (ambulatory competent; AC), patients with self-reported functional weakness (ambulatory weakness; AW), patients dependent on walking aids (ambulatory assistance; AA), and patients dependent on a wheelchair(WC).

Results: A total of 88 healthy controls and 110 patients with neuromuscular disease were recruited. Most patients were diagnosed with mitochondrial disease $(\mathrm{n}=61)$. The PJP in HC $(19.1 \mathrm{~W} / \mathrm{kg} \pm 0.7)$ was not different compared with AC (17.2
$\mathrm{W} / \mathrm{kg} \pm 1.1, p<0.275)$; however, AW (9.9 W/kg \pm 0.7$)$, AA $(5.7 \mathrm{~W} / \mathrm{kg} \pm 1.1)$ and $\mathrm{WC}(2.3 \mathrm{~W} / \mathrm{kg} \pm 0.6)$ followed a stepwise decline in PJP as ambulatory capacity decreased $(p<0.01)$. This trend persisted across the age range of our subjects (controls; 3-65 years, patients; 4-83 years of age), demonstrating that PJP and ambulatory capacity are related.

Conclusions: PJP is an easy-to-perform, quantitative measure of ambulatory ability in patients with neuromuscular diseases including mitochondrial disease. PJP scores clearly distinguish patients who require different types of ambulatory assistance.

Source of funding: ACHRF, ACHRI, MitoCanada

\section{It's about more than just baby teeth: An examination of early oral care in Canada}

Leonard Smith ${ }^{1}$

Larry Katz ${ }^{2}$

Herbert Emery ${ }^{3}$

Jackie Sieppert ${ }^{4}$

Zoe Polsky

Kimberly Nagan ${ }^{6}$

1 Department of Pediatrics in the Faculty of Medicine, University of Calgary, Calgary, $\mathrm{AB}$

2 Faculty of Kinesiology, University of Calgary, Calgary, AB

${ }^{3}$ Department of Economics, University of Calgary, Calgary, $\mathrm{AB}$

${ }^{4}$ Faculty of Social Work, University of Calgary, Calgary, AB

${ }^{5}$ Kinesiology, McGill University, Montreal, QC

${ }^{6}$ Research Associate, University of Calgary, Calgary, AB

Background: It may come as a surprise to medical practitioners that the most common infectious disease in young children is dental decay and that oral health is the most prevalent unmet healthcare need of children. Children who present with early childhood caries (ECC) can suffer from pain, sleeplessness, malnutrition, failure to thrive and toxic stress. ECC has been dismissed by the medical system as a dental problem not a bealth problem, and service providers have failed to appreciate the vital link between oral health in children and overall wellbeing.

Several actions (or inactions) on multiple levels interact to create an environment that allows for poor oral health in a child. A conceptual model has been developed which proposes that children's oral health is influenced on three levels: child, family, and community. What follows are suggestions to preventing ECC on all three levels. 
Preventative strategies: Prevention of ECC on a child-level includes simple, low-cost tasks such as daily wiping of gums and brushing teeth with an appropriate sized toothbrush and a small amount of fluoridated toothpaste, reducing the amount of sugary foods consumed and eliminating the use of bottles and/or no-spill cups of milk, formula or juice in bed. A visit to a dentist by the first birthday to assess the oral condition and prevent progression if decay is present is the current recommendation made by the Canadian Dental Association as delayed first dental visits are directly correlated with increases in ECC. On a family level, important influences to address include the health status of the parents, socioeconomic status (SES), social support, coping skills, physical safety, culture and health behaviors and practices. Community- wide prevention comes in the form of interdisciplinary collaboration, public health education programs and public policy at the various levels of government. Community-wide services should include preventative education and treatment from non-dental professionals such as primary care physicians, nurses and social workers. As such a minor amount of time is required to educate health professionals in identifying early oral deficiencies; continuing education is a viable avenue for training with the assistance of dental professionals. In addition to screening for oral health disease, doctors can take this opportunity to educate parents on the risk factors for caries even before a child's teeth begin to erupt. Greater collaboration between medical and oral health professionals is needed to provide adequate, holistic care.

Conclusion: Uniquely to this particular infectious disease, ECC can be almost completely eliminated through prevention. Furthermore, prevention comes at little cost to families and the healthcare system; therefore, the benefits of shifting funding from tertiary restoration to primary prevention should be highlighted. Educational efforts, community awareness and changes in public policies are required, as ECC remains a complex problem necessitating multifaceted intervention.

\section{Cardiovascular responses to standing in older adults: How do the prospective data measure up?}

\author{
Brett H. Shaw ${ }^{1,2}$ \\ Thomas M. Loughin ${ }^{3}$ \\ Stephen N. Robinovitch ${ }^{1}$ \\ Victoria E. Claydon ${ }^{1}$ \\ 1 Department of Biomedical Physiology and Kinesiology, \\ Simon Fraser University, Burnaby, BC \\ 2 Cumming School of Medicine, University of Calgary, \\ Calgary, $\mathrm{AB}$ \\ ${ }^{3}$ Department of Statistics and Actuarial Science, Simon Fraser \\ University, Burnaby, BC
}

Background: Orthostatic hypotension refers to a significant decline in blood pressure when upright. It has a high incidence and prevalence in older adults, and represents a potential risk factor for falls in these individuals. We showed previously that in long-term care residents, fallers have larger delayed orthostatic declines in systolic arterial pressure, as well as poorer recovery of systolic arterial pressure compared with non-fallers. Fallers also showed greater orthostatic declines in systolic cerebral blood flow velocity, a potential downstream mechanism linking orthostatic hypotension and falls. These analyses were done using retrospective falling data. In the present study we aimed to assess whether the relationships between cardiovascular responses to orthostasis and falling are maintained when using prospective fallingrisk.

Methods: Elderly residents $(\mathrm{n}=59)$ in two long-term care facilities underwent a passive seated orthostatic stress test, and beat-to-beat blood pressure and cerebral blood flow velocity responses were assessed throughout testing. Other risk factors for falls were ascertained from patient medical records. Falling history was collected from facility records over the subsequent year. Cardiovascular responses to orthostasis were compared between fallers ( $\geq 1$ fall) and non-fallers. We compared fallers and non-fallers using the Student's t-test or Wilcoxon rank test.

Results: After one year of follow-up, 45 (age range: 66-94 years) residents remained in the long-term care facilities: 32 were classified as fallers; and 13 as non-fallers. Fallers were more likely to use a walker or wheelchair and to have had a prior stroke. Supine systolic arterial pressure was significantly higher in non-fallers compared with fallers, despite no significant differences in medication use. Fallers achieved a lower nadir systolic arterial blood pressure when moved upright during the consensus (0-3 min) and delayed (3-15 $\mathrm{min})$ periods of testing. The recovery of systolic arterial pressure 
was also significantly more impaired across multiple time intervals in fallers compared with non- fallers. Finally, fallers reached a lower nadir cerebral blood flow velocity in the delayed period. There were, however, no differences in the absolute change in systolic arterial pressure, or cerebral blood flow velocity.

Conclusions: In this prospective study, we found that older adults in long-term care continue to display impairments in systolic arterial pressure and cerebral blood flow velocity that are worse in fallers. These data confirm our earlier observations that in older adults in long-term care, impaired cardiovascular responses to orthostatic stress are associated with an increased risk of falling.

\section{Serum Infliximab levels following first infliximab dose for induction of remission in children with ulcerative colitis}

Mariel Van Woudenberg ${ }^{1}$
Iwona Wrobel ${ }^{1}$
Hien Huynh $^{2}$
Kevan Jacobson $^{3}$
Wael El-Matary $^{4}$
Cynthia Seow $^{5}$
Remo Panaccione $^{5}$
Jennifer deBruyn $^{1}$

1 Division of Pediatric Gastroenterology, Department of Pediatrics, Faculty of Medicine, University of Calgary, Alberta Children's Hospital, Edmonton, AB

2 Division of Pediatric Gastroenterology, Department of Pediatrics, Stollery Children's Hospital, University of Alberta, Edmonton, $\mathrm{AB}$

3 Division of Pediatric Gastroenterology, Department of Pediatrics, British Columbia Children's Hospital, University of British Columbia, Vancouver, BC

4 Division of Pediatric Gastroenterology, Department of Pediatrics, University of Manitoba

5 Division of Gastroenterology, Department of Medicine, Faculty of Medicine, University of Calgary, Foothills Medical Center

Introduction: Infliximab (IFX) is an important therapy in children with ulcerative colitis (UC). It is effective as a rescue therapy in children who fail to respond to conventional corticosteroid therapy; however, up to $39 \%$ of children do not demonstrate an adequate initial response or subsequently lose response and still require colectomy. Serum IFX levels have been shown to correlate with clinical outcomes in inflammatory bowel disease. Limited studies have been conducted in children with UC. An improved understanding of serum IFX levels in children with UC is essential and may lead to future strategies of optimizing response to IFX. The aim of this study is to evaluate the relationship between serum IFX levels at Weeks 1,2 and 6 and clinical outcomes of clinical remission and response at Week 8 in children with UC.

Methods: This is a multicenter prospective observational study of children with UC who commenced IFX therapy at the Alberta Children's Hospital (Calgary), Stollery Children's Hospital (Edmonton), British Columbia Children's Hospital (Vancouver) and Children's Hospital (Winnipeg) between March 1, 2013 and September 1, 2014. Baseline data was collected on demographics, current and past medication use, disease classification and activity (Pediatric Ulcerative Colitis Activity Index [PUCAI]) and growth parameters. Patients received IFX according to standard induction and maintenance protocol: $5 \mathrm{mg} / \mathrm{kg}$ intravenous infusion Weeks 0 , 2, 6 (induction) followed by infusions every 8 weeks thereafter (maintenance) with corticosteroid premedication. Serum IFX levels were drawn at Week 0 (peak), Week 1, Week 2 (trough) and Week 6 (trough). PUCAI scores were recorded at Weeks 1, 2, 6 and 8. Clinical remission was defined by PUCAI $<10$ and clinical response was defined by a decrease in PUCAI score of at least 20 from baseline to Week 8 .

Results: A total of 19 children (eight males, 11 females) were included in the current analysis. The median age of enrolment was 13.7 years, the median age of diagnosis was 13.1 years and the median time from diagnosis to enrolment was one year. Indication for starting IFX therapy was acute severe UC in 10 children and chronic active UC in nine children. Past and current medications included 5-aminosalicylic acid, steroids, Imuran/Azathioprine, methotrexate and others. Median IFX dose was $300 \mathrm{mg}$ at Weeks 0, 2 and 6. Median time between the first and second dose was 14 days, and 28 days between the second and third dose.

Median IFX levels were $199.2 \mu \mathrm{g} / \mathrm{ml}, 62.1 \mu \mathrm{g} / \mathrm{ml}, 50.3$ $\mu \mathrm{g} / \mathrm{ml}$ and $46.7 \mu \mathrm{g} / \mathrm{ml}$ at Week 0 (peak), 1, 2 (trough) and 6 (trough), respectively. Based on our outcome criteria, $71 \%$ of patients were in clinical remission and 59\% demonstrated clinical response by Week 8. Only one subject required colectomy and none developed antibodies.

Conclusion: This study demonstrates promising outcomes in pediatric patients with UC who initiate Infliximab therapy for induction of remission. Further work needs to be done to correlate IFX levels with clinical outcomes in these patients. 


\section{Narcotic and Benzodiazepine agreements and monitoring: An implementation 'how-to'}

\author{
Jacqueline Williamson \\ Tracy Burton \\ Beverly Burton \\ Jared Van Bussel \\ Jeff Brockman \\ Cathy Scrimshaw \\ Gavin Parker \\ John Rottger \\ Tobias Gelber
}

*All authors contributed and should be recognized equally

Introduction: There have been over 50 opiate-related deaths in southwestern Alberta in the first quarter of 2015, with the media focusing specifically on the aboriginal reserves in this area. Opiate prescription in Alberta is monitored by the provincial Triplicate Program, but as a medication with increasing tolerance with use, physicians can easily find themselves prescribing long-term opioids often in increasing doses. In large quantities, they have significant implications on the respiratory drive and can easily lead to loss of consciousness and death. These medications also have a high street value and can be diverted to supplement income to low-income households. What process can be implemented to ensure adequate pain control for those who need it, and decrease the number of diverted medications?

Methods: The Associate Clinic physicians and staff drafted a new Narcotic and Benzodiazepine Agreement (NBA) based on published examples. The electronic medical records (EMR) were used to identify the number of people prescribed opioids or benzodiazepines and flagged to renew their NBA at their next visit. Random urine drug screening (UDS) was implemented on one randomly-selected day each week for anyone on an NBA. UDS was used to determine compliance and illicit drug use. Number of NBAs due, new NBAs signed and UDS samples were tracked and qualitative discussions were had with staff and physicians regarding the implementation of this policy. Regular practice redesign meetings were held to discuss the process and preliminary numbers to trouble-shoot issues. Consensus was sought from all meeting participants before new process changes were implemented.

Results: Prior to implementing the new policy, there were 665 people receiving long-term opioids in the last 12 months, averaging to 13.4 booked appointments per day of patients on narcotics. There were 331 narcotic contracts that had been signed, ever. There had been 15 UDS samples in the month of May 2015. In July 2015, following implementation of the policy, there were 452 patients seen in clinic with narcotic contracts due for renewal, 30 of which were completed, and 19 UDS samples were taken. In August 2015, there were 367 patients seen in clinic with narcotic contracts due, nine of which were completed, and 25 UDS samples were taken.

Qualitative analysis of the process uncovered physician discretion for patient exclusion from the process due to opioid use for cancer-related pain, palliative care and extremely low usage. Inclusion and exclusion criteria were honed and UDS process was further developed to ensure patient privacy, patient protection and efficiency. We identified that EMR data is only as useful as the information that goes into it and many medication lists were not up to date, resulting in falsely-elevated patient numbers. Regular patient pain evaluations were valuable for identifying any benefit or lack of benefit to narcotic use, and to open a discussion about supplementary and alternative pain management strategies.

Conclusion: Narcotics are a useful tool to combat acute pain, when used long-term in chronic pain they can lead to increased dependency and may be used for recreational purposes. There are many other modalities to tackle chronic pain that can be used in tandem with, or in place of, narcotics. NBAs and regular pain scale monitoring are valuable tools to appropriately follow a patients progress and adjust treatment accordingly. UDS without judgement should be used to ensure appropriate use of these medications. NBAs and UDS protocols require process evaluation, clear definitions of inclusion and exclusion criteria to ensure a consistent approach. Our goal is to reduce harm to the population as a whole by managing the appropriate use of narcotics within the community. This is a process that can be done effectively on a small scale. Until implemented and monitored on a large scale, population wide effects cannot be appreciated.

\section{Professionalism and disruptive behaviour in healthcare}

Michael Arget

Janet de Groot ${ }^{2}$

${ }^{1}$ Undergraduate Medical Education

${ }^{2}$ Equity and Professionalism Office, Cumming School of Medicine, University of Calgary, Calgary, AB

Purpose: The purpose of this study was twofold: to highlight the effect of disruptive behaviour on professionalism and patient safety; and, to explore leading ways to measure 
disruptive behaviour and highlight successful programs that have been developed.

Introduction/background: Professionalism is a concept emphasized throughout the trajectory of a medical career, When one looks to define professionalism, there are policies, competencies, frameworks and codes of conduct that attempt to direct physicians, but these tend to be high level, often aspirational and without specific direction on how to act in a professional manner. The converse, disruptive behaviour, is defined as: "Any inappropriate behavior, confrontation, or conflict, ranging from verbal abuse to physical and sexual harassment."

Methods: This project started with a meeting of the Professionalism Scholarship group in the Cumming School of Medicine in Calgary. Content experts in the field were identified and their work was explored. Literature searches on physician conflict were completed with the assistance of the Health Sciences Librarian.

Findings: Disruptive behaviour has an adverse impact on health human resources and patient safety. Review of the literature resulted in very little information about disruptive behaviour between staff physicians, but well documented between various other health professionals groups. Mechanisms to quantify disruptive behaviour include unsolicited patient complaints and metrics that look at workplace culture like the Safety Attitudes Questionnaire. The Medical School Graduation questionnaire is an instrument that provides information about learner mistreatment, including disruptive behaviour. Leading programs exist to work on reducing disruptive behaviour among physicians. One successful program addresses disruptive behaviour directly, with an awareness and peer support program for high-risk physicians. Other programs aim to improve workplace culture by focusing on non-technical skills improvement, such as the Comprehensive Unit-based Safety Program (CUSP).

Future directions: Metrics to quantify professionalism are being developed for individual behaviour. Organizational interventions, such as CUSP (and Team Steps), focus on teamwork skills to enhance the culture of professionalism. In future, integrating measurement and response to unprofessional behaviour and organizational interventions require evaluation of their capacity to reduce disruptive behaviour and improve patient safety.
Can we use administrative data to define an emergency department population at risk for pulmonary embolism? Development of an algorithm of presenting complaints and test ordering to define a target population

Kristin Burles ${ }^{1}$

Dongmei Wang2

Kevin Senior ${ }^{2}$

Eddy Lang2

Andrew McRae ${ }^{2}$

${ }^{1}$ Faculty of Medicine, University of Calgary, Calgary, $\mathrm{AB}$

2 Department of Emergency Medicine, Alberta Health Services, Calgary, AB

Introduction: Administrative data is a valuable tool for clinical research purposes; it can be used to monitor disease trends and appropriateness in test ordering, and can inform operational decisions and health policies. Many diseases have been studied using administrative data, and most research has relied on the use of diagnostic codes, as defined by the International Statistical Classification of Diseases and Related Health Problems, 10th Revision (ICD-10). Pulmonary embolism (PE) is a potentially life-threatening condition that is considered in the differential diagnosis of many cardiopulmonary presentations, and can be a difficult diagnostic challenge. As such, clinical research on PE diagnostic strategies remains important. Two ICD- 10 codes exist for PE (I26.0 and I26.9); however, studying this disease remains challenging since no diagnostic code exists for suspected PE. The purpose of our work was to develop an algorithm of administrative data that can be used to define a population at risk for PE, thus aiding in clinical research and quality improvement for this group of patients.

Methods: Hospital administrative data from the Calgary region were obtained for patients over the age of 18 years presenting to the emergency department from July 2013 to January 2015. Medical charts, computerized tomographic angiography (CTA) reports and/or ventilation perfusion (V/Q) scan reports were reviewed to confirm the diagnosis of PE. To develop an algorithm for identifying patients at risk for $\mathrm{PE}$, the fraction of patients diagnosed with PE for each presenting complaint to the emergency department was determined. Only patients undergoing D-dimer testing and/or a CT scan and or a V/Q scan were considered. Combinations of presenting complaints capturing the greatest numbers of 
PEs were assessed using sensitivity, specificity and positive predictive value calculations.

Results: By including patients whose presenting complaint was Cardiac Pain, Chest Pain (Cardiac Features), Chest Pain (Non-Cardiac Features), Shortness of Breath, Syncope/Pre-syncope, Hemoptysis, or Unilateral Swollen $\mathrm{Limb} / \mathrm{Pain}$, in combination with D-dimer testing and/or a CT scan and or a V/Q scan, we captured PE diagnoses with a sensitivity of $77 \%$ and a specificity of $87 \%$ (95\% CI 0.7426-0.7944). The positive predictive value was 0.807 . We also evaluated the use of ICD-10 codes for identifying patients diagnosed with PE. Of 1,453 patients with PE, 46 were improperly coded and should have been assigned an alternative code. Another 211 patients were miscoded, and did not have a $\mathrm{PE}$, although their exact diagnosis remained to be determined. Thus, 257 patients were falsely recorded in hospital administrative database as being diagnosed with a PE, resulting in a miscoding rate of $17.7 \%$ for I 26.0 and I26.9 codes.

Conclusions: Our algorithm can be used to identify patients at risk for PE with reasonable specificity and sensitivity; however, caution should be exercised when using administrative data for studying $\mathrm{PE}$, and a strategy that includes review of medical records to validate the accuracy of ICD-10 coding is recommended. This work will be used to monitor the appropriateness of imaging for PE.

\section{A novel use of a point-of-view camera for teaching lateral canthotomy and cantholysis to emergency physician trainees}

\section{Stephanie Cote ${ }^{1}$ \\ Karim Punja ${ }^{2}$ \\ Kevin Warrian ${ }^{2}$ \\ Adrian Gooi ${ }^{3}$ \\ Patrick Gooi ${ }^{2}$}

1 Faculty of Medicine, Cumming School of Medicine, University of Calgary Alberta Health Services

2 Division of Ophthalmology, University of Calgary Alberta Health Services

${ }^{3}$ Department of Otolaryngology-Head and Neck Surgery, University of Manitoba, Winnipeg, MB

Introduction: Orbital compartment syndrome (OCS) is a vision threatening ocular emergency that occurs when there is a sudden rise in orbital pressure resulting in damage to intraocular structures. Lateral canthotomy and cantholysis
(LCC) is a simple procedure used to decompress the orbit. Emergency physicians should be comfortable evaluating, diagnosing and performing a LCC to manage an OCS to decrease the risk of vision loss in the event that consultation and intervention by an ophthalmologist is not possible in a timely manner. Developing this skill is challenging, as this procedure is seldom performed; therefore, resources need to be available. Current training videos are an excellent learning tool but are limited by several factors, such as not capturing from the perspective of the physician performing the procedure. Point-of-view (POV) cameras show the physician's perspective, which is more conducive to training as it mimics the experience for trainees. We report our novel technique of recording a LCC using a head-mounted POV camera as a resource for emergency physician trainees in learning this procedure.

Methods: A head mounted POV GoPro Hero 4 Silver camera (GoPro, San Mateo, CA, U.S.A.) with a modified $5.4 \mathrm{~mm} \mathrm{f/2.5}$ aftermarket lens with a $60^{\circ}$ field of view (Peau Productions Inc, San Diego, CA, U.S.A.) as used. This lens was pre-focused to a working distance of 17 inches, set to $1080 \mathrm{P}$ on narrow recording at 48 frames per second, and had spot metering and the low light functions turned on. The camera functions were controlled remotely by an assistant with the use of GoPro App on a tablet computer to ensure proper framing of the camera.

Results: The recording system was effective in capturing the surgical field, fine detail of the procedure and positioning of the instruments. The instructive teaching of the LCC procedure was done simultaneously during the surgery filming, with minor editing post-surgery.

Conclusion: Point-of-view cameras have great potential in assisting the education at the post-graduate level within residency training programs. The POV recording system can be a training device in an emergency setting for performing a LCC or other procedures that emergency physicians may seldom encounter. Video recording from the physician's perspective simulates the experience for trainees and could leave them feeling more confident in their ability to perform the procedure. 
Optimal shift duration for emergency physician efficiency, effectiveness and safety: A comparison of 6, 7 and 8-hour shifts

\author{
Michele Foster ${ }^{1,2}$ \\ Zhankun Sun ${ }^{1,3}$ \\ Dongmei Wang 1 \\ Grant Innes 1,5 \\ Laurie-Ann Baker ${ }^{6}$ \\ Andrew $\mathrm{McRae}^{7}$ \\ Eddy Lang ${ }^{1,4}$ \\ ${ }^{1}$ University of Calgary \\ 2 Department of Medical Sciences \\ ${ }^{3}$ Haskayne School of Business \\ ${ }^{4}$ Academic and Clinical Department Head for Emergency \\ Medicine \\ ${ }^{5}$ Research Director, Department of Emergency Medicine \\ ${ }^{6}$ Zone Deputy Department Head, Operational Lead, Calgary \\ Zone, Alberta Health Services \\ 7 Clinician-Scientist and Research Director, Department of \\ Emergency Medicine, University of Calgary and Alberta \\ Health Services
}

Introduction: Emergency departments (ED) require 24-hour physician coverage, and must develop a schedule that balances patient safety with physician efficiency and preferences. There are no studies comparing shift length in regards to safety, metrics and productivity. Our objective is to determine if there is a difference in the efficiency of patients seen per hour between 6,7, or 8-hour shifts in the ED. Secondary outcomes include the number of patient handovers, and unscheduled ED revisit rates associated with different shift lengths.

Methods: This retrospective study is based on one urban ED, where 81 physicians provided care for 79,941 visits during the study period (2013/09/01 to 2014/08/31). Forty-one physicians met our inclusion criteria; working a minimum of 30 shifts of varying lengths over this period. Minor treatment shifts were excluded from analysis. Administrative data and an online scheduling system was used to compare scheduled shift length and number of patients seen per hour as well as percentage of patients handed over to the next physician from the total seen that shift, and return visits within 72 hours. One-way analysis of variance and t-tests were used to compare the means between three different shift types.

Results: A total of 3,214 shifts of varying start times (1,467 6-hour shifts, 531 7-hour shifts and 1,216 8-hour shifts) were included. Mean start times for 6,7 and 8-hour shifts were
12:00, 17:00 and 10:00 respectively. The average number of patients seen per hour for 6-, 7- and 8-hour shifts was 2.56 (95\%CI 2.53-2.59), 2.75 (95\%CI 2.68-2.82) and 2.50 (95\%CI $2.47-2.53)$, respectively ( $<<0.001$ for comparison of 7 - against 6- and 8-hour shifts). The average handover rate for 6-, 7-and 8-hour shifts was $22.14 \%$ (95\%CI 21.50-22.78), $27.45 \%$ (95\%CI 26.37-28.53) and $17.36 \%$ (95\%CI 16.86-17.87), respectively ( $p<0.001$ for the comparison of 8 - against 6 - and 7-hour shifts). All night shifts in this ED are 7-hours; a limitation of these results is the inclusion of night shifts, which have higher handover rates. There was no significant difference between shifts for 72-hour return rates.

Conclusion: In this comparison, 7-hour shifts offer optimal physician efficiency, while 8-hour shifts may be more desirable from a safety perspective, as fewer handovers mean fewer opportunities for error. Higher efficiency means reduced wait times, increased patient flow and fewer adverse events in the ED waiting room.

\section{The surgical management of malrotation: A Canadian Association of Pediatric Surgeons survey}

Ceilidh Kinlin ${ }^{1}$

Anna C. Shawyer ${ }^{2}$

1 Cumming School of Medicine, University of Calgary, Calgary, $\mathrm{AB}$

${ }^{2}$ Alberta Children's Hospital, Calgary, AB

Background: Intestinal malrotation, present in approximately $0.2-1 \%$ of the population, puts the patient at risk for volvulus, bowel obstruction and massive intestinal loss and requires urgent surgical correction. With the advent of laparoscopy, more surgeons are utilizing this approach in place of the traditional open "Ladd's procedure". Laparoscopic advantages are decreased risk of infection, decreased cost, increased surgical visualization and precision and smaller incisions. Although typically a benefit of laparoscopy, the lack of surgical adhesions has been theorized as a risk for post-operative or recurrent volvulus.

Objective: To examine practice patterns of pediatric surgeons for the management of stable patients with malrotation.

Methods: A pre-piloted survey was distributed at the 2015 Canadian Association of Pediatric Surgeons annual meeting. Descriptive statistics were calculated.

Results: The response rate for the survey was 35\% (150 distributed, 52 returned). Most institutions (39.5\%) saw, on average, 5-10 cases of malrotation per year. Most respondents 
(54.2\%) indicated that the laparoscopic and open Ladd's procedure were considered equal surgical approaches for stable patients. The respondents were nearly equally divided (47.9\% yes; $44.7 \%$ no) with respect to whether laparoscopic Ladd's led to a higher risk of post-operative or recurrent volvulus. Of those who answered yes, most indicated that an increased risk of volvulus was due to an inadequate widening of mesentery (43.4\%). All participants who indicated a standard open approach believed that there was a higher risk of post-operative or recurrent volvulus with laparoscopic Ladd's, although several respondents who indicated a different standard surgical approach also held thisbelief.

Conclusion: Despite the low response rate, the results of this study highlight a lack of consensus in the surgical approach to intestinal malrotation, which is in keeping with the lack of clear recommendations from the available literature. Collaboration via a multi-center observational study could provide the basis for consensus for this uncommon, but serious entity.

\section{Cyberbullying physicians and the impact on empathy}

Veronique Dorais Ram

University of Calgary, Calgary, $\mathrm{AB}$

Introduction: Cyberbullying and online rating sites have become a prominent media topic. According to the Oxford English Dictionary, it entails: "the use of information technology to bully a person by sending or posting text or images of an intimidating or threatening nature." With the increase in technology, information has become harder to monitor and control. The line between what constitutes constructive criticism and intimidating bashing remains skewed. What happens when patients employ social media to do more than evaluate their experience or satisfaction with health care providers? For instance, a rural town of northern Alberta $(<10,000)$ has a Facebook page employed to report which physician is on-call, who to avoid, who will give out drugs and to vent about dissatisfaction with decisions made by doctors in complex care situations. What is the impact of such forums on the patient-physician relationship? Can such sites impact physician empathy, welfare, autonomy and social justice?

Methods: This project is divided into two parts: a literature review and systematic analysis of studies on the ethical principles for physician rating sites and other forms of social media; and, a series of interviews with physicians on their experiences with social media and its impact on their sense of empathy.

Results: Literature review using serial terms in PubMed, OVID Embase, OVID Medline, and Google Scholar. Search terms were as follows: 1) "cyberbullying" OR "cyber bullying"; 2) "physicians" AND “patients"; 3 ) “empathy”; 1) AND 2); and, 1) AND 2) AND 3).

\begin{tabular}{l|c|c|c|c}
\hline & PubMed & $\begin{array}{c}\text { OVID } \\
\text { Embase }\end{array}$ & $\begin{array}{c}\text { OVID } \\
\text { Medline }\end{array}$ & $\begin{array}{c}\text { Google } \\
\text { Scholar }\end{array}$ \\
\hline 1 & 3427 & 339 & 266 & 25,100 \\
\hline 2 & 114,932 & 145,593 & 125,193 & $2,120,000$ \\
\hline 3 & 17,547 & 17,721 & 13,746 & 892,000 \\
\hline 4 & 16 & 2 & 1 & 818 \\
\hline 5 & 0 & 0 & 0 & 222 \\
\hline
\end{tabular}

Conclusions: There is an increase in the number of publications on the potential benefits and harms of social media and ranking sites with regards to patient evaluations of physicians; however, direct evidence remains limited. Given the subjective nature of the topic, measures will remain qualitative in nature and therefore, the second part of this project will interview physicians to examine the impact of inflammatory and incriminating commentaries on physician empathy and wellness. The aim is to employ the results to offer strategies for quality standards and regulation of physician-related websites to maintain the transparency of the health care system without stripping both patients and physicians of their humanity.

\section{Medicine in Sci-Fi: Our hopes and fears}

Adam Shumate

\section{University of Calgary Cumming School of Medicine}

Introduction: It is a well-established phenomenon that patient expectations and perceptions can influence health outcomes in measurable ways. Although it may be impossible to predict the expectations of individual patients, larger societal trends may be gleaned by observing popular media. Regardless of whether mass media is the product of public opinion or the other way around, by watching for common trends we can identify themes that exist in the public subconscious. To identify subject matter specific to the future 
of medicine source material was chosen not from modern portrayals of our current medical establishments, but the imaginary futures presented in the Science Fiction genre.

Methods: A list of the top 100 grossing films at domestic box offices was obtained from www.boxofficemojo.com for each year of the last decade (2004-2013), totaling 1,000 titles. Films on this list whose genre were not identified as Science Fiction, and whose synopsis on the Internet Movie Database (www.imdb.com) that had no indications of fictional technology were omitted, leaving 170 entities. Films that were poorly received were considered to be less likely to have had significant contributions to the public mindset, thus any film with a rating below $50 \%$ by either the public audience or film critics on www.rottentomatoes.com were omitted. Eighty-six films passed the criteria for minimal acclaim, however 11 of these were excluded based on an assessment of their synopsis. Seventy-five films proceeded to full analysis, which consisted of a critical viewing by the author with focus on the aforementioned topics of medical technology, future medical issues and interactions with healthcare systems. Twenty-four films did not yield any relevant findings. The findings from 51 films were reviewed for common themes.

Results: Major themes identified about future medical issues include (in arbitrary order): Creative Innovation: regardless of the particulars, it is expected that we will invent new technology, from handheld gadgets to body-encasing pods that will drastically alter our medical capabilities; Unequal Access: large populations will not have access to medical care or new innovations; Imaging: internal structures will be clearly identifiable to layman eyes, often in colour-coded 3D; Mad Science: cutting edge research gets performed in a moral vacuum with no respect to ethics or common sense; Man-Machine Interface: the boundary of human physical and mental limitation will be expanded, but at the expense of horrendous invasion of body and mind; Loss of Self: by improving humans we may lose sight of humanity, many biological and technological means may have the ability to undermine our individuality; Zombies: genetics have replaced voodoo as the dark force best not meddled with; The Apocalypse: whether through best intentions or poor judgment, global catastrophe could arise unexpectedly from an obscure laboratory.

Conclusion: Various pervasive themes in popular media about the future of medicine may be present in the subconscious expectations of patients, and have the potential to influence their interactions with modern healthcare.

\section{The effects of fuzzy trace decision-making on estimation of post-test probabilities in medical students}

Alec Campbell
Jolene Haws
Pietro Ravani
Kevin Busche
Kevin McLaughlin
University of Calgary, Calgary, AB

Introduction: Accurate diagnosis is a keystone of optimal healthcare and diagnostic errors have been identified as a major cause of preventable morbidity and mortality in Canada. Most clinicians initially learn to diagnose using simple heuristics (i.e., how closely the patient's presentation fits with an idealized version of disease), which are extremely susceptible to base-rate neglect resulting in overestimation of rare disease prevalence. Many diagnostic strategies have been proposed to reduce this bias, among them mathematical models such as Bayesian analysis (the gold standard) as well as hybrid strategies such as improper linear modelling (ILM). We hypothesized that ILM would allow medical students to correctly diagnose fictional cases as accurately as Bayesian analysis with less mental effort.

Methods: Twenty-nine second-year University of Calgary medical students were randomized to ILM vs. Bayesian groups. Both groups received nearly identical test booklets, containing four cases asking the student to evaluate the probability of presence of a fictitious disease and the mental effort needed for this evaluation, based on a referral letter and appropriate background information. Booklets differed slightly in the amount of information supplied; the Bayesian group received likelihood ratios for each clinical sign of each disease, while the ILM group was only told which of the seven clinical signs provided was most sensitive and most specific. Cases differed in the likelihood of disease presence and the prevalence of each disease.

Results: No statistically significant difference in diagnostic accuracy, cognitive load or time required to finish was observed between the two groups. Both groups consistently overestimated the probability of a rare disease.

Conclusion: In addition to the lack of difference between the two groups, it was also noted that both groups vastly overestimated the probability of rare disease presence. These results suggest that despite being directed to use ILM or Bayesian analysis, students reverted to using heuristics when forced to commit to a diagnosis. 
The use of a policy dialogue to facilitate evidence-informed policy development for improved access to care: The case of the Winnipeg Central Intake Service (WCIS) for total joint replacement surgery of the hip and knee

\author{
Zaheed Damani ${ }^{1}$ \\ Gail MacKean ${ }^{1}$ \\ Eric Bohm² \\ Brie DeMone ${ }^{3}$ \\ Brock Wright ${ }^{4}$ \\ Tom Noseworthy ${ }^{1}$ \\ Jayna Holroyd-Leduc ${ }^{1}$ \\ Deborah A. Marshall ${ }^{1}$ \\ ${ }^{1}$ Department of Community Health Sciences, University of \\ Calgary, Calgary, $\mathrm{AB}$ \\ 2 Department of Surgery, University of Manitoba, Winnipeg, \\ $\mathrm{MB}$ \\ 3 Manitoba Health, Healthy Living and Seniors, Acute, \\ Tertiary and Specialty Care, Winnipeg, MB \\ ${ }^{4}$ Winnipeg Regional Health Authority, Winnipeg, MB
}

Introduction: Engagement processes such as policy dialogues are critical for the development of responsive, effective and sustainable, evidence-informed policy. The Winnipeg Central Intake Service (WCIS), a single-entry model (SEM) was introduced in 2012 in Winnipeg, MB to improve patient access to hip and knee replacement surgery. Findings from our evaluation of this model were used to develop five policy directions to help improve access to scheduled services across Manitoba. We hosted a Policy Roundtable Meeting, designed using elements of best practice for deliberative dialogues, to discuss these policy directions.

Methods: The five policy directions were centred on the following: 1) measurement and monitoring of quality; 2) central intake as a preferred model for service delivery; 3 ) provincial scope; 4) transparent processes and performance indicators; and 5) patient choice of provider. Fifty individuals were invited by the research team; 44 attended from five stakeholder groups-patients, family physicians, orthopedic surgeons, surgical office assistants and WCIS team members from across Manitoba-to participate. Through facilitated sessions, groups were asked to answer two overarching questions based on their experiences: 1) Do you agree in principle with the policy direction?; and 2) Should the policy directions be pursued? Responses from discussions and evaluation forms were analyzed, to identify key themes and trends.

Results: Though there was strong support, it was not universal for all policy directions-they were prioritized and refined to be more responsive and inclusive. Four key findings are clear from participant responses, engagement and summative evaluations. Firstly, participants felt that the Roundtable Meeting achieved its purpose and was useful. Secondly, participant engagement and enthusiasm suggests that the policy directions resonated with the stakeholders and that their consideration will be critical for the improvement of access to scheduled and clinical services in Manitoba. Thirdly, our tailored adaptation of the key design elements for deliberative processes worked well for the discussion of issues related to access to care. Finally, our decision-maker research partners' expectations were exceeded, demonstrating the effect of aligned objectives, close work with research teams and co-development of the policy directions.

Conclusion: There was keen interest in centralized intake for scheduled services from all health regions in Manitoba. Although supportive, stakeholder groups wished to see further, more rigorous use and evaluation of SEMs. Across healthcare, and in access improvement in particular, a true deliberative policy dialogue process holds promise for creating an environment that enables all of these voices to be heard, and for effective, evidence-informed policy as a result.

\section{Flipping the undergraduate medical education classroom to challenge students in LGBTQ health}

Stephanie Hudson ${ }^{1}$

Shoghi Nikoo ${ }^{1,2}$

Kira Ableson ${ }^{1}$

Marguerite Heyns ${ }^{1}$

Joe Raiche ${ }^{1}$

Murray Lee ${ }^{1}$

${ }^{1}$ Cumming School of Medicine

${ }^{2}$ Department of Sociology, University of Calgary, Calgary, AB

Introduction: Despite recent improvements in public policy regarding lesbian, gay, bisexual, trans and queer (LGBTQ) issues, LGBTQ people continue to endure high burdens of poor health in Canada. A lack of cultural sensitivity around LGBTQ health issues within the Canadian healthcare system is one of the drivers of this inequity. A Stanford University School of Medicine study published in Journal of the American Medical Association found that there are on average 
five hours dedicated to LGBTQ health over entire North American medical curricula; it is, therefore, no surprise that a 2005-2006 AAMC survey found that 50 percent of medical students felt they had insufficient training to care for LGBTQ patients. The purpose of this pilot change to the University of Calgary's Population Health curriculum was to improve education on LGBTQ health by employing a flipped classroom model to promote critical thinking and active learning.

Methods: A collaboration of students and faculty at the University of Calgary, Cumming School of Medicine designed a four-part audio podcast series that introduced basic terms and concepts, explained how phobias, stereotypes and discrimination negatively impact health, and described current initiatives and research in LGBTQ health. Enrolled students reviewed the audio series prior to attending a mandatory large group session. During the large group session, students were posed ambiguous multiple-choice questions and encouraged to respond to one another's answers.

Results: Students were engaged during group discussion and provided thoughtful answers that reflected familiarity with material presented in the audio series. The facilitated discussion challenged students to critically reflect on their learning, subsequently demonstrating an enhanced understanding of the concepts and issues introduced in the audio series. Initial feedback suggests the flipped classroom model to be a valuable tool.

Conclusion: The flipped classroom model of undergraduate medical education appears to be an effective way to build medical students' basic competence in discussing LGBTQ issues and understanding challenges in LGBTQ health. Further evaluation of this education method will help guide future curricular restructuring, thus increasing cultural sensitivity among Canadian physicians and increasing the capacity of the Canadian healthcare system to provide culturally sensitive care to LGBTQ people.

\section{Should Primary Care Networks cover foot care for diabetics in Alberta?}

Maninder Longowal ${ }^{1}$

Daniel Edgcumbe ${ }^{2}$

${ }^{1}$ Cumming School of Medicine

2 Department of Family Medicine, University of Calgary, Calgary, $\mathrm{AB}$

Introduction: Many Primary Care Networks (PCNs), especially those in rural areas, have large proportions of aging diabetic patients seen in multidisciplinary diabetes clinics. These patients are often unable to manage their own foot care, or cannot afford the $\$ 35$ charge per session of foot care, which can lead to further neuropathic damage, diabetic foot ulcers (DFUs), infections and amputation. With funds distributed on a per-patient basis, PCNs often have to make difficult decisions regarding where to allocate the limited resources. This project aimed to analyze both cost-effectiveness data and high quality articles to make a recommendation as to which subsets of patients should be offered free foot care.

Methods: The literature selection was performed on September 6, 2015 by searching Pubmed, OVID Medline, and Cochrane Central Register of Controlled Trials for the terms "diabetes foot care", "diabetes foot ulcer", "diabetes foot ulcer cost" and "diabetes foot ulcer prognosis". Another search was performed in Google Scholar for "Alberta diabetes statistics".

Results: Markov Model simulation of three theoretical groups of 10,000 patient cohorts showed that management of the diabetic foot according to guideline-based care improves survival, reduces diabetic foot complications, is cost-effective and even cost-saving compared with usual care. Each of these models predicted a cost of $<\$ 25,000$ per Quality Adjusted Life Year obtained, which is considered to be a cost-effective threshold for policy makers. Furthermore, by risk stratifying patients using the International Working Group on the Diabetic Foot (IWGDF) risk classification system, physicians can identify those in whom foot care is most likely to prevent a DFU: those with peripheral neuropathy and peripheral vascular disease or foot deformity (category 2 ); or those with a previous ulcer or amputation (category 3 ).

Conclusion: The evidence suggests that PCNs would best utilize limited resources by implementing the 2015 IWGDF Risk Classification System to categorize ulceration and infection risk in diabetic patients. Those in category 2 and 3 who receive scheduled foot care at 3-6 months and 1-3 months, respectively, had greatly reduced rates of amputation and ulceration compared with lower risk patients. Data 
showed that providing free foot care for these patients may be cost-saving for the health care system.

\section{Creation and implementation of a peer mentorship program for Leaders in Medicine: initial experiences}

\author{
Nabeela Nathoo ${ }^{1}$ \\ Craig A. Beers ${ }^{1}$ \\ Bryan G. Yipp ${ }^{2}$ \\ Morley Hollenberg ${ }^{2}$ \\ Paul L. Beck ${ }^{3}$ \\ ${ }^{1}$ Hotchkiss Brain Institute \\ ${ }^{2}$ Snyder Institute of Chronic Diseases \\ ${ }^{3}$ Department of Medicine, University of Calgary. Calgary, AB
}

Introduction: The Leaders in Medicine (LIM) program at the University of Calgary provides students with the opportunity to pursue a graduate degree (Master's, $\mathrm{PhD}$ ) in conjunction with the MD program. To aid in navigating through the program and its various educational sessions (e.g., journal clubs and research-in- progress seminars) and to help create a well-developed cohort of future clinician-researchers, a peer mentorship program was proposed for joint degree students in LIM.

Methods: Students newly admitted to the joint program in LIM in $2015 \quad(n=19)$ were contacted by the Program Administrator to determine the following: 1) if they were interested in having a mentor; and 2) what attributes they were looking for in a mentor. Senior students in the joint program in LIM (admitted in 2014 or earlier) $(n=49)$ were also contacted by the Program Administrator to determine the following: 1) if they were willing to be a mentor; and 2) what attributes they felt they could offer to a potential mentee. Attributes listed for both mentors and mentees were restricted to a maximum of three items.

Results: Just under half of newly admitted students (9/19) requested a mentor (six $\mathrm{MD} / \mathrm{PhD}$ and three $\mathrm{MD} / \mathrm{MSc}$ ). Similarly, just under half of senior LIM students (22/49) volunteered to be mentors (19 MD/PhD, two MD/MSc and one $\mathrm{MD} / \mathrm{MBA}$ ). Attributes listed by those who requested to be mentees and those who volunteered to be mentors were compared, and those whose attributes appeared to align best were paired up. Introductions between mentors and mentees took place during a LIM social event in September 2015. In instances where either the mentee or mentor could not be present to meet face-to-face, introductions were carried out via email.
Conclusion: A peer mentorship program in the context of a highly specialized program such as LIM may greatly benefit all members of the program. To help optimize mentor/mentee relationships, the "One Minute Mentor" document previously created by Dr. Mitchell Feldman at the University of California (San Francisco) has been adapted for LIM and will be available to LIM members to facilitate meetings between mentors and mentees. Future directions include hosting mentorship workshops and assessing the usefulness and impact of the mentor/mentee relationship through short surveys.

\section{What impact does academic mentoring have on subsequent academic performance in medical school?}

Monika Oliver, $\mathrm{BSc}^{1}$

Hatem Alnassar, $\mathrm{MD}^{2}$

Sita Gourishankar, MD MSc ${ }^{2}$

${ }^{1}$ University of Calgary, Calgary, $\mathrm{AB}$

2 University of Alberta. Edmonton, $\mathrm{AB}$

Introduction: Academic mentoring (AM) is considered a valuable aspect of undergraduate medical education at the University of Alberta. Historically, our undergraduate program has not had a formal AM program for students experiencing academic difficulty. Subsequently, a faculty member was appointed to proactively and systematically identify struggling learners and offer one-on-one AM with the goal of improving subsequent performance.

Methods: A retrospective cohort study was preformed, examining the impact of $\mathrm{AM}$ on academic performance of undergraduate medical students. Seventeen students (years 14) were flagged for academic difficulty as measured by a score of $<60 \%$ on mandatory course work. Students were asked to complete a preliminary questionnaire with subsections on self-perception as a learner, sources of stress, school-life balance, study methods, and prior educational background. Results were used to guide discussion in a subsequent 60-90 minute mandatory AM session with the AM Coordinator. Success of the AM was measured by the need for further remediation for continued academic struggle following initial intervention.

Results: Of the seventeen participants, eleven (64.70\%) experienced repeat performance deficiencies. Of this subset, ten students were noted to have $>1$ subsequent failure, suggesting persistent academic struggles. All participants expressed appreciation for the opportunity to discuss their 
performance candidly with a faculty member; the majority reported being satisfied with the AM session.

Conclusion: Academic mentoring, as a component of remediation, is a new initiative at the University of Alberta. The high percentage of students with persistent struggles following an initial mentoring session suggests an ongoing need that requires faculty attention. The positive feedback from students implies targeted mentorship is a worthwhile component of successful remediation.

\section{Canadian Association of Emergency Physicians 2015 Academic Symposium: Current state and recommendations for improvement for funding emergency medicine academic units}

\author{
Eddy S. Lang, $\mathrm{MD}^{1}$ \\ Jennifer D. Artz, $\mathrm{PhD}^{2}$ \\ Ryan D. Wilkie, $\mathrm{BSc}^{3}$ \\ Ian G. Stiell, MD MSc4 \\ Claude Topping, $\mathrm{MD}^{5}$ \\ Francois P. Belanger, $\mathrm{MD}^{6}$ \\ Marc Afilalo, $\mathrm{MD}^{7}$ \\ Tia Renouf, $\mathrm{MD}^{8}$ \\ Anthony Crocco, $\mathrm{MD}^{9}$ \\ Kelly Wyatt, BA ${ }^{2}$ \\ Jim Christenson, MD ${ }^{10}$
}

${ }^{1}$ Department of Emergency Medicine, University of Calgary, Calgary, $\mathrm{AB}$

2 Canadian Association of Emergency Physicians (CAEP), Ottawa, ON

${ }^{3}$ Faculty of Medicine, University of Calgary

${ }^{4}$ Ottawa Hospital Research Institute, University of Ottawa

5 Faculty of Medicine, Université Laval, Québec City, QC

${ }^{6}$ Alberta Health Services

${ }^{7}$ Faculty of Medicine, McGill University, Montréal, QC

${ }^{8}$ Discipline of Emergency Medicine, Memorial University of Newfoundland. St. John's, NL

9 Department of Pediatrics, Division of Emergency Medicine, McMaster University, Hamilton, ON

${ }^{10}$ Department of Emergency Medicine, University of British Columbia, Vancouver, BC

Introduction: Emergency medicine (EM) is a burgeoning academic medical discipline that struggles for equitable recognition of its contributions to the health care system. We set out to describe the current state of academic EM funding in
Canada and to develop recommendations for improving its support. Like other disciplines, EM requires the appropriate academic and research support to ensure that it can deliver high quality health care to patients. Healthy academic funding is critical to providing growth in the three key pillars of any medical discipline: undergraduate education, post-graduate education and research. This funding must be aligned with the mission and goals of the medical school and the regional health authority, with the ultimate objective of enhancing emergency care delivery and patient outcomes.

Methods: A panel of eight leaders from different EM academic units in Canada was assembled. To develop our recommendations, we used a number of distinct approaches, including conducting a literature review, surveying current EM academic heads, as well as incorporating data from a prior environmental scan. Recommendations were drafted and presented at an academic symposium, where attendee feedback was incorporated. The draft recommendations were then distributed to the Canadian Association of Emergency Physicians (CAEP) Academic Section for feedback before being finalized.

Results: Overall, ten recommendations were developed by the panel to address funding challenges and recommend solutions experienced by EM academic programs across Canada. A strategic plan was seen as integral to the success of an EM unit, especially when aligned with departmental and institutional priorities. A business plan, occasionally overlooked, was deemed an important component for planning and sustaining the academic mission. A number of recommendations surrounding philanthropy were included to create partnerships with existing foundations and to engage multiple stakeholders and communities. Synergy between academic and clinical EM departments was also viewed as an opportunity to strengthen both missions. Education and networking for current and future members was also seen as invaluable to ensure the continued success of our academic missions through developing strongleadership.

Conclusions: The panel felt strongly that these recommendations could improve the financial circumstances for many Canadian EM units. There is a considerable wealth of resources that can contribute to financial stability for academic units and an annual networking meeting in conjunction with continuing education on these issues will be important for future success. 


\section{Accessibility of medically-appropriate nutrition for clients of Calgary poverty support agencies}

Emily Macphail

Cumming School of Medicine, University of Calgary, Calgary, $\mathrm{AB}$

Introduction: Nutrition is an integral component of wellbeing; however, for those who have particular medical conditions (e.g. allergies, celiac disease, diabetes, hypertension) or are in specific life stages (e.g., childhood, pregnancy), it becomes even more important in the management of their health for them to have access to particular types of food. Other unavoidable dietary restrictions (e.g., religious-based, requirement for soft foods, etc.) may also decrease one's ability to access sufficient nutrition, further compromising health outcomes.

Individuals experiencing poverty often have poorer health than their counterparts with higher socioeconomic status, while at the same time, also facing greater barriers to addressing their health concerns. Following medically-required diets with particular restrictions often necessitates foods that are more expensive or less readily available, thereby compounding the health impacts of poverty. Because a variety of organizations and agencies in Calgary provide nutrition support for those living in poverty, an exploration of the dietary requirements and restrictions that they are able to provide for was felt to be indicated.

Methods: A sample of agencies within Calgary who provide food to those living in poverty were contacted and asked a series of questions regarding whether the meals that they serve are required to meet certain nutrition standards (and if so, how these were determined), if they are able to accommodate various dietary restrictions and needs and, if so, which ones and in what manner. Inquiries were also made around accommodation of non-medical dietary restrictions (e.g., vegetarianism, religiously-based, etc.). If an agency was unable to accommodate a particular dietary restriction or need, the obstacles to doing so were explored.

Results: Access to medically-appropriate nutrition and to diets accommodating particular non- medical restrictions is variable across different Calgary agencies that provide individuals living in poverty with nutrition support. Barriers to such vary between agencies, but common themes include time and resource constraints.

Conclusion: For health care professionals working with patients who are struggling financially, it is important to pay attention not only to education regarding dietary requirements and health impacts, but also to the patient's resources with regards to their ability to access appropriate nutrition for their condition. To improve health outcomes for those living in poverty, support agencies may require additional funding or other resource, making advocacy work in this area a consideration.

\section{Understanding evidence-to-practice gaps in upper gastrointestinal bleeding management in Albertan emergency departments through qualitative focus groups: Developing a guide}

Shoghi Nikoo ${ }^{1,2}$

Marilynne Hebert ${ }^{3}$

Daniel Grigat ${ }^{4}$

Catherene Joseph ${ }^{4}$

Gilaad Kaplan ${ }^{3,5}$

Kerri Novack ${ }^{6}$

Ian Surdhar ${ }^{7}$

Eddy Lang1,4

1 Cumming School of Medicine, University of Calgary, Calgary, $\mathrm{AB}$

2 Department of Sociology, University of Calgary, Calgary, AB

${ }^{3}$ Department of Community Health Sciences, University of Calgary, Calgary, $\mathrm{AB}$

4 Emergency Strategic Clinical Network, Alberta Health Services

${ }^{5}$ Department of Medicine, University of Calgary, Calgary, AB

${ }^{6}$ Division of Gastroenterology, University of Calgary, Calgary, $\mathrm{AB}$

7 Winnipeg Regional Health Authority, Winnipeg, MB

Introduction: Between 2012-2014, an average of 6,347 patients presented annually to emergency departments in Alberta with upper gastrointestinal (GI) bleeding; representing a provincial health system burden of over 20,000 inpatient bed days, at an estimated operating cost of over $\$ 16$ million. Alberta provincial administrative data and an audit of the Calgary Zone reveal significant opportunities to improve the management of nonvariceal upper GI bleed (NVUGIB). Data indicate substantial variation $(28 \%-76 \%)$ between sites in the admission of NVUGIB, with $8.7 \%$ of admissions being for fewer than 24 hours, suggesting some stable patients are admitted in order to access endoscopy resources that could be provided on an outpatient basis. Furthermore, $71 \%$ of transfusions were administered to patients with hemoglobin 
levels above $70 \mathrm{~g} / \mathrm{L}$ and $29 \%$ to patients above $90 \mathrm{~g} / \mathrm{L}$. Aggressive management of NVUGIB can expose patients to risks associated with admission, endoscopy and transfusion, which might be avoided in stable low-risk patients through application of evidence-based clinical guidelines. This ongoing project investigates current physician behaviour in risk stratification and disposition of patients, endoscopic management and red cell transfusion through qualitative focus groups.

Methods: Because of their ability to elicit rich data from the perspectives of groups under study, focus groups are often used in social science and behavioural research to understand factors that drive behaviour. Until recently, however, focus groups have been used relatively infrequently to understand barriers to clinical practice guideline adoption. The Theoretical Domains Framework provides a validated approach to understanding behaviour and designing behavioural interventions. Building on this avenue of investigation, this project adapts the Theoretical Domains Framework to develop a focus group guide relevant to emergency practice in Alberta.

Results: The focus group guide incorporates validated domains of behavioural change into a ranking exercise and activity-based discussion. The ranking exercise focuses subsequent examination of a NVUGIB case and discussion of how, when and why evidence-based recommendations and tools are or are not used in practice. Domains explored include knowledge/skills, social/professional role and identity, beliefs about capabilities and consequences, reinforcement, goals and environmental context and resources.

Conclusion: By eliciting rich data characteristic of focus group research, the authors expect to understand current practice and develop a clinical pathway for NVUGIB that reduces patient risks and healthcare costs.

\section{A retrospective chart review identifying burn injuries treated from the suspected manufacturing of hash oil at Foothills Hospital Medical Centre}

Vincent Gabriel ${ }^{1}$

Kyle Ricord ${ }^{2}$

Patricia Harasym ${ }^{3}$

1 Division of Physical Medicine and Rehabilitation, Departments of Clinical Neurosciences, Pediatrics and Surgery

2 Cumming School of Medicine

3 University of Calgary, Calgary, AB

Background: Burn injuries sustained during the illegal manufacturing of hash oil often constitute major burns that require significant surgery and prolonged burn care. The incidence of these burns are likely to increase due to the increased legalization of marijuana, producing leftover lower grade leaf and stalk material that can then be used to make hash oil, despite the inherent risk of doing so. Burn units should be aware of the blast mechanism involved in these burn injuries and the population it is affecting. This awareness will contribute to modifications of burn treatment management and expectations. We report the incidence, demographics and outcomes of such burns treated at the Burn Unit of Foothills Medical Complex (FMC), Calgary, Alberta.

Methods: A retrospective chart review of all burn admissions treated at FMC between 2010 and 2015 was performed for evidence or suspicion of burns sustained during the manufacturing of illegal drugs; in particular, hash oil. Total burn admissions treated during period of study are represented graphically; including the number of patients, their age group and gender. The same data for patients sustaining burns from hash oil manufacturing are also represented. Individual cases (represented by case number) are tablated; detailing mechanism, area of body affected, burn depth, total body surface area affected, admission duration, treatment type and outcome.

Results: Of the adult burn patients $(\mathrm{n}=161)$ admitted over the 24-month review period, 12 (7.5\%) had burns sustained as a result of an explosion during illegal drug manufacturing. Nine out of the 12 patients (75\%) were under the age of 41 years and $83 \%$ were male. Body areas suffering the most burns were the face and neck (occurring in 11 patients), followed by the hands (occurring in nine patients). Three patients sustained inhalation injuries with one patient succumbing to his injuries. Face and neck burns accounted for the highest percentage of body part affected in burn injuries, with both being $67 \%$. The 
average burn body surface area was $23.5 \%$ (range 3\%-70\%). The average length of admission was 31.7 days (range 3-69 days). Nine of the twelve patients in the study group required debridement and split skin grafting.

Conclusion: Twelve cases of burns related to hash oil manufacturing were extrapolated from records representing $7.5 \%$ of all adult burn admissions treated at FMC during the 24 month investigation period. The majority of patients were young males with facial and neck burns. There was a greater severity of burn injuries sustained during the illegal manufacturing of hash oil affecting a greater average body surface area and requiring a longer length of admission than in the general population.

\section{Health promotion and youth empowerment in West Bengal, India}

Amrita Roy ${ }^{1,2}$

Rupayan Roy ${ }^{3}$

${ }^{1}$ Department of Community Health Sciences, University of Calgary, Calgary, $\mathrm{AB}$

${ }^{2}$ Leaders in Medicine program, Cumming School of Medicine, University ofCalgary, Calgary, $\mathrm{AB}$

3 Department of English Language and Literature, Queen's University, Kingston, ON

Background: The health-promotion function of public health is the process of "enabling people to increase control over, and to improve, their health", using strategies of building healthy public policy, creating supportive environments, strengthening community action, developing personal skills, and reorienting health services. Youth in West Bengal, India face a number of challenges in this regard, including restrictive tradition-based societal norms and clashing cultural mores in globalization-era India, disempowerment from age-based and other social power hierarchies and a lack of easy access to accurate information and effective resources.

Research questions: This mixed-methods study addressed three research questions. First, among youth, what is the level and nature of access to knowledge and resources concerning health and wellness, human rights and youth empowerment? Second, to what extent are opportunities for the above prioritized in school and community policies? Third, what are the current attitudes and beliefs of youth on these issues?

Methods: Focus-group interviews and surveys were conducted with a total of 298 secondary and postsecondary students during July and August 2006, in Kolkata (Calcutta) and the neighbouring Hooghly and Howrah districts of the state of West Bengal. Personal interviews were also conducted with 10 educators and one public official. Bilingual (Bengali and English) semi-structured interview guides and survey questionnaires were designed and pilot-tested before use. Interviews were audio-recorded with permission, transcribed, and coded for a thematic analysis. Answers from the survey questionnaires were entered into Excel, and descriptive statistical analyses were conducted using the software Stata.

Results: Answers to closed-ended survey questions revealed several gaps in knowledge on health and social issues, particularly among females and younger teens; the latter likely reflects stricter messaging to, and enhanced disempowerment of, these groups. Youth participants reported relying primarily on the media and on their peers for information on health and social issues; $88 \%$ ranked the media as their primary source of information. They openly recognized the poor quality and unreliability of the information they were receiving, with $84 \%$ indicating dissatisfaction. There was particular frustration among the youth on the lack of open discussion around sexuality and sexual health, even within the context of the then-new sex education program in state secondary schools. Youth spoke passionately about wanting to participate in bringing about change in their society, to address the health and social issues facing them and their communities; however, they expressed frustration at the that barriers youth like them faced in this regard.

Conclusions: This research suggests that youth need and want opportunities to access accurate information, meaningful resources and effective programs pertaining to health and social issues affecting themselves and their communities. Moreover, youth need and want concrete opportunities to build their capacity to contribute meaningfully to addressing these issues, at both the individual and the community levels. Such opportunities can help foster individual-level health promotion through heightened individual empowerment. Additionally, empowerment of youth can also allow them to become agents of broader social change in their communities, leading ultimately to improved population health outcomes. 
Quality improvement in the ICU: Reducing delirium through the use of non-benzodiazepine sedatives

\section{Dana Saleh \\ Marnee Wilson}

\section{University of Toronto, Toronto, ON}

Introduction: The incidence of delirium among mechanically-ventilated intensive care unit (ICU) patients is alarmingly high; reaching $83 \%$ and increasing mortality and healthcare costs. Latest delirium prevention and safety guidelines recommend the use of non-benzodiazepine sedatives such as Dexmedetomidine or Propofol to achieve sedation goals in mechanically-ventilated adults. The aim of this initiative is to decrease the incidence of delirium at the South Health Campus ICU by 30\% by January 2018 through the adoption of Dexmedetomidine or Propofol as the preferred sedative infusions in ventilated patients.

The initiative's team would include representatives from medicine, nursing, respiratory therapy, pharmacy and quality management, as well as RN stakeholders. The initiative is driven by the Institute for Healthcare Improvement's Plan ("Do, Study, and Act Cycle") in which the team predicts that the use of non-benzodiazepine infusions will reduce delirium rates. The medical student will document staff compliance and delirium rates from patient records using the Intensive Care Delirium Screening Checklist worksheet (ICDSC).

Methods: The team will design and deliver six, two-hour educational sessions on delirium prevention throughout August and September 2016 to all ICU staff and senior administrators, as measured via attendance audits. During the trial period of October-November 2016, Intensivists and Nurse Practitioners (NPs) will be encouraged to prescribe Dexmedetomidine or Propofol for at least twenty ventilated patients requiring sedation; alcohol withdrawal cases will be excluded. During the Do phase, the team will document and analyze observations and delirium rates, comparing them to the initial prediction and revising the plan for the second test cycle. The final plan will be implemented in January of 2017January 2018 and will be evaluated using a quasi-experimental one-group pretest-posttest design with plans for dissemination, thereby emphasizing the impact of collaborative practice in the ICU between medicine and nursing and its role in improving the therapeutic management of ventilated adult patients.

Project stakeholders include ICU staff, nursing and medical providers, the Canadian ICU Collaborative Faculty (CICF) and families. Facilitating factors include the expertise of the site's medical and nursing clinicians and mentorship from the CICF. Identified barriers include resistance to the increased costs associated with Dexmedetomidine, the influx of novice nurses and other care providers into critical care and noncompliance with the completion of the daily goals forms formulated during ICU rounds. Potential risks to patients from sedatives include hemodynamic instability.

Future direction: This proposal is currently in the planning phase and could be revised post-review by ICU management and other stakeholders in January of 2016.

\section{Patient beliefs and perceptions surrounding obesity management: A parallel qualitative study}

Emma Skolnik

Aric Sudicky

Ian Mitchell

Cumming School of Medicine, University of Calgary, Calgary, $\mathrm{AB}$

Background: Obesity continues to be one of the leading causes of morbidity and mortality worldwide as 1.5 billion people are estimated to be overweight. Despite the increasing rate of obesity in Canada, the success of clinical weight loss interventions is low, thus highlighting the importance of research in this area. Effective communication plays a critical role in any therapeutic process, and as primary care providers, family physicians are in an optimal position to treat medical concerns related to obesity. Given the vulnerable nature surrounding weight management, it is imperative that research focuses on understanding patient perspectives to optimize the success of counselling. The purpose of this study was two-fold: firstly, to gain insight from adult and pediatric patient perceptions in regards to their preferences surrounding current primary care obesity counselling; and secondly, to develop practical learning points to aid family physicians in the setting of clinical weight management.

Case presentation: The study incorporated two qualitative interview-based case studies, including one adult and one adolescent patient $(\mathrm{n}=2)$. Interviews involved standardized questions, which gave subjects the opportunity to discuss their experiences in the Canadian medical system. Obese adult patients were classified as over 18 years of age (BMI >30), while obese adolescents were classified between 13 and 18 years of age (99th percentile weight category).Qualitative findings were categorized into three themes: personal beliefs 
and body image; determinants of comfort in clinical settings; and perception of doctors as prevention resources.

Conclusion: From both arms of the study, subjects felt that weight concerns were not addressed frequently enough by family physicians. Reminders involving negative aspects of obesity and associated health consequences were not an effective counselling tactic. Both adult and adolescent participants suggested that patient-centred weight management counselling should be approached with a sense of comfort, honesty and adaptability. The adolescent subject emphasized the importance of interacting with physicians by means of direct conversations, involving a sense of understanding and individualization. The results highlight the importance of understanding patient perspectives and concerns while appreciating the specific needs of diverse patient populations. The discussions raised are valuable and set the stage for thoughtful and clinically-meaningful research in the field of weight management medicine. Future work will consider increased patient numbers and patients across centres, to evaluate the robustness of the themes identified in these interviews.

\section{Calgaryrefugeehealth.ca: Website model for refugee health resource hubs across Canada}

\author{
Varun Suresh ${ }^{1}$ \\ Haotian Wang ${ }^{1}$ \\ Manmish Bawa ${ }^{1}$ \\ Anna Lee Coakley ${ }^{1,2}$ \\ 1 Cumming School of Medicine, University of Calgary, \\ Calgary, $\mathrm{AB}$ \\ ${ }^{2}$ Mosaic Refugee Health Clinic, Calgary, AB
}

Introduction: Approximately 300 government-assisted and 400 private-sponsored refugees and 150 refugee claimants arrive in Calgary annually. There are many services available to assist refugees with health and social issues but access to relevant information is difficult. Physicians who assist refugees face similar accessibility issues. Refugeehealth.ca is a website that serves as an information hub where refugees and their physicians in the Greater Vancouver region can find information on healthcare and other services. We have launched a similar resource in Calgary to bridge the gap in the access of information for both the refugee population and their physicians.
Design principles: The website was built on three core pillars: user-friendly platform; the provision of relevant information and resources; and the provision of information in multiple languages. We made the website easy to use, simple to navigate with clearly defined subheadings and mobile friendly.

Methods: Since most of the background information related to refugee populations is consistent between Calgary and Vancouver, we have linked our website to refugeehealth.ca. Information specific to Calgary, like the locations of the refugee clinics, are posted on the website with embedded maps. To make the website multilingual, Google Translate was embedded allowing the site to be translated to different languages. Costs of this project was kept low by using Squarespace, a user-friendly but powerful website-builder and by dedicating student project time rather than hiring help.

Future steps: Currently, the website is online and running, but it will need constant refinement and evaluation to become a better resource. We are in talks to run trials of the website within the refugee and family doctor population.

\section{Targeting leukemia stem cells in the development of novel therapeutics for refractory infant leukemia: A case report}

Lacey Brennan

Justin Riemer

Aru Narendran

POETIC Laboratory for Pre-Clinical and Drug Discovery Studies, and the Division of Pediatric Oncology, Alberta Children Hospital, Faculty of Medicine, University of Calgary, Calgary, $\mathrm{AB}$

Background: Although treatment strategies for pediatric Acute Lymphoblastic Leukemia (ALL) have improved event-free survival (EFS $>85 \%$ ), relapse rates in certain subgroups such as infant ALL (EFS 23\%-51\%) remain unacceptably high. The high incidence of relapse implies the existence of a subset of rare, persistent cells that are not affected by current treatments. These treatment-resistant cells have been termed leukemia stem cells (LSCs). In many ways, LSCs resemble normal hematopoietic stem cells, including their ability to remain quiescent, self-renew and generate substantial numbers of differentiated leukemic progeny. These attributes provide LSCs with the ability to maintain a low, but critical number of cancer stem cells to effectively sustain the disease. These properties may also explain how LSCs elude 
aggressive chemotherapy and remain as minimal residual disease, eventually repopulating the patient during relapse. We hypothesize that targeting therapeutics to LSCs may decrease relapse rates in infant leukemia.

Case presentation: A nine-month-old male infant presented with an elevated white blood cell count. Upon further investigation, he was diagnosed with ALL for which he was treated with the conventional multidrug chemotherapy regimen according to Children's Oncology Group (COG) protocol. He went into remission for three months until his first relapse. He then underwent stem cell transplantation; however, after a brief remission, the leukemia recurred and he ultimately succumbed to the disease. During the last relapse, a bone marrow sample was collected following local ethics board approval and parental consent. From this specimen, LSCs were purified using stem cell markers and fluorescence-activated cell sorting (FACS). Gene expression and transcriptome analyses were carried out to identify aberrant growth stimulatory pathways and unique therapeutic targets in these cells.

Conclusion: We propose that this approach can be used in the future to target LSCs upon initial patient presentation. Manipulating these pathways early in the disease course may lead to improved patient outcomes and lower rates of relapse.

\section{Reducing infection transmission in the playroom: Balancing patient safety and family-centred care}

\section{Allana Ivany, BScN, RN, CIC ${ }^{1}$ \\ Chantal LeBlanc, BPs, CCLS 2 \\ Mackenzie Grisdale, BA, MAc, \\ Bridget Maxwell, BN, RN, CIC ${ }^{1}$ \\ Joanne M. Langley, MD, MSc ${ }^{1,2,4}$}

1 Infection Prevention and Control Services, Dalhousie University, Halifax, NS

2 Child Life Services, IWK Health Centre, Dalhousie University, Halifax, NS

${ }^{3}$ Dalhousie University, Halifax, NS

4 Departments of Pediatrics and Community Health and Epidemiology, Faculty of Medicine, Dalhousie University, Halifax, NS

Background: Family-centred care requires that institutions develop strategies to allow sibling visitors to hospitalized children while reducing risks of infectious disease transmission. Most guidelines recommend that siblings not be permitted to visit playrooms. This approach was not seen as consistent with family-centred care in our setting; therefore, in a pilot project we developed an approach for screening siblings with cooperation of families, child life specialists, the care team and the infection prevention and control service.

Methods: A literature review using CINAHL and PubMed databases (Medical Subject Heading terms: visitors to patients, child, infection, nosocomial and siblings) from 2004-2014 did not uncover formal established methods for reducing playroom infectious disease exposures. Benchmarking with other Canadian centers revealed a diversity of approaches. Child life, the ward staff and infection prevention and control at this center collaborated to develop a sibling screening strategy.

Results: The collaborative approach led to a process based on a screening form that is introduced to the family during admission. The process requires the cooperation of the admitting nurse, parents and child life staff. In the first two years of the project, approximately $10 \%$ of screened siblings had a potentially communicable illness.

Conclusion: A collaborative multidisciplinary approach, based on family centered care principles, led to a process whereby siblings of hospitalized children can be allowed to visit playrooms, while reducing risk of infectious disease transmission.

\section{Dermatofibrosarcoma protuberans of the parotid gland: report of a rare case and review of the literature}

Ashley V. Hinther ${ }^{1}$

Morris Kostiuk, $\mathrm{PhD}^{2}$

Jeffrey Harris, MD, MHA, FRCSC 2

Vincent L. Biron, MD, PhD, FRCSC ${ }^{2}$

1 Undergraduate Medical Education, Cumming School of Medicine, University of Calgary, Calgary, AB

2 Division of Otolaryngology-Head and Neck Surgery, University of Alberta Hospital, Edmonton, AB

Background: Dermatofibrosarcoma protuberans (DFSP) is a rare soft tissue sarcoma that most frequently affects the dermis and subcutaneous tissue. DFSP most commonly occurs on the trunk, however $\sim 18 \%$ of DFSP cases occur in the head and neck region, and rarely in the parotid region. DFSP is a slow growing, indolent tumour. DFSP is characteristically locally aggressive, with a low propensity for metastases. The optimal treatment for DFSP is wide circumferential surgical excision with histologically-negative margins, due to its characteristically locally aggressive nature and asymmetric extension in the dermal stroma. Given the rarity of this 
tumour involving the parotid gland it is important to differentiate it from the more commonly occurring myoepithelial tumours in this location; however, this can be a challenging pathologic diagnosis especially at the time of intraoperative frozen section analysis. We therefore aimed to further characterize this lesion with a panel of molecular markers using digital droplet polymerase chain reaction.

Case presentation: A 58-year-old male presented with a 4 x 5 $\mathrm{cm}$ solid nodule over his right parotid gland. DFSP was not considered within the differential diagnosis from fine needle aspirate biopsy but given the rapid growth of this lesion over the preceeding two months, surgical excision was planned and expedited. Two intraoperative frozen section biopsies were taken and reviewed with the pathologist. The frozen sections demonstrated high amounts of cellularity, and a storiform pattern with spindle cells. An intraoperative diagnosis favoured carcinoma with subcutaneous extension. A more extensive surgical procedure was therefore performed to include a total parotidectomy, ipsilateral selective neck dissection and wide local excision of skin overlying the lesion. The patient recovered well from the surgery with excellent return of function of his facial nerve and at the time of publication was six months disease-free. The final pathology reports showed short spindle cells with high cellularity and pleomorphism, and were subtyped as cellular dermatofibrosarcoma protuberans with rare adipose and glandular element invasion. No further treatment was recommended following discussion by a multidisciplinary team. Further molecular characterization with gene expression profiling of this lesion was performed, with final results pending.

Conclusion: The treatment remains the same for DFSP regardless of the origin: wide local excision of the tumour with margins of $2.5-3.5 \mathrm{~cm}$.

\section{Stroke and systemic embolic infarct in a patient with hypereosinophilia: A case report}

Gordon AE Jewett ${ }^{1}$

Carol Tham ${ }^{2}$

Bijoy K Menon²

${ }^{1}$ Faculty of Medicine

2 Department of Clinical Neurosciences, University of Calgary, Calgary, $\mathrm{AB}$

Background: Hypereosinophilia has been reported in association with ischemic stroke in a small number of cases.
Several mechanisms have been postulated for the etiology of stoke in hypereosinophilia with little consensus. We report the case of a patient who presented with systemic embolic infarcts including stroke, splinter hemorrhages and myocardial emboli in the setting of hypereosinophilia with no intra-arterial thrombus identified and no definitive diagnosis of vasculitis.

Case presentation: A 56 year-old right hand-dominant man presented with transient confusion, left hemiparesis and splinter hemorrhages that developed overnight. He had minimal deficits with a National Institutes of Health Stroke Scale of 1 . Initial investigations revealed a significantly elevated high sensitivity Troponin $\mathrm{T}(472 \mathrm{ng} / \mathrm{L})$ and hypereosinophilia $\left(10.4 \times 10^{9} / \mathrm{L}\right)$. Magnetic resonance imaging (MRI) revealed multiple areas of acute infarct in all cerebral vascular territories. Cardiac MRI revealed multiple lesions in the septum consistent with embolic infarct but there was no evidence of endomyocardial fibrosis. No thrombus was identified with transesophageal echocardiogram or MRI and no ongoing active emboli were seen with transcranial Doppler imaging. The patient declined a bone marrow biopsy. Skin biopsy was not in keeping with a working diagnosis of eosinophilic granulomatosis with polyangiitis. Cutaneous microthrombi were seen leading to presumptive diagnosis of hypereosinophilic syndrome.

Conclusion: The patient was treated with IV methylprednisolone resulting in rapid normalization of eosinophilia. Low dose acetylsalicylic acid was started and no further embolic phenomena were detected at 3-weeks following presentation. There was no consensus diagnosis. There appears to be an association between hypereosinophilia and stroke in some patients. The mechanism for this process remains poorly understood.

\section{Quantifying supine leg movement with respect to normal walking gait: A case report}

Adrienne Kline

Payam Zandiyeh

Janet Ronsky

Schulich School of Engineering, University of Calgary, Calgary, $\mathrm{AB}$

Introduction: Walking is fundamental to a healthy quality of life in humans as it is both a means of exercise and a mode of transportation. The execution of normal walking is an intricate sensorimotor task involving the interactions of both dynamic and balancing neurological processes. To study this 
interplay appropriately, concurrently-gathered data from body movement and brain activity needs to occur. Functional MRI (fMRI) technology can be utilized to achieve this; however, current fMRI technology requires subjects to be in supine position during data collection. Thus, the similarities between supine-generated walking movements and normal walking need to be clearly understood. Currently, very little work has been done investigating biomechanics of supine movement, and how these movements relate to those of upright walking biomechanics. These extant studies on lower limbs use different methodologies and show mixed results. The majority of research surrounding the biomechanics of supine movement has focused on applications involving weightless exercise (i.e.; space travel; zero gravity). Thus, the purpose of this pilot study was to compare mathematical models of the biomechanical signatures of supine knee flexion with that of executed walking. Standing knee flexion was assessed as well as supine knee flexion because normal walking occurs with the body in a vertical position. The expectation was that supine leg movements would mimic a portion of an individual's natural gait cycle. The findings will help to determine the veracity of the assumption that neurological data collected in an fMRI scanner from supine leg movements will provide comparable neurological data to that of normal walking.

Methods: One healthy male subject (age, 24 years) took part in this pilot study. As this was a single-participant study, random error variance across subject could not be ascertained. To determine the degree to which between-participant error might affect the findings, random error was introduced into the models and their effects assessed. The subject performed two tasks: upright normal walking gait; and supine knee bends on a training table, both in a lab setting. Each task was performed five times to gain consistency across trials. Pearson correlation coefficients were used to determine the degree of similarity between the two conditions. Lastly, a mathematical/biomechanical model was developed to represent the movements executed.

Results: Pearson correlation coefficients were calculated to compare normal walking knee flexion to standing knee flexion and supine knee flexion. It was determined that walking compared with standing knee flexion showed a low to moderate effect $(\mathrm{r}=0.2276)$, and was statistically significant $(\mathrm{p}<0.0228)$. Walking compared with supine knee flexion showed a very strong, significant effect $(\mathrm{r}=0.8973)$, and was statistically significant $\left(\mathrm{p}<1.377 \times 10^{-36}\right)$.

Conclusion: Based on the data collected, it can be concluded that supine knee flexion and adduction strongly mimics walking knee flexion and adduction, supporting the rationale of using fMRI neurological data based on supine knee flexion. The models showed a robustness regarding random error variance. Specifically, right vs. left knee flexion showed a higher level of variation than when a single trial for right knee flexion had superimposed noise in it. For the trial with noise added to the right knee flexion had higher levels of variation than that associated with average right knee flexion across five trials. This finding indicates that multiple trials are useful in generating reliable overall measures.

\section{Progressive ataxia with palatal tremor: A case report}

Ryan MacIsaac ${ }^{1}$

Justyna R. Sarna ${ }^{1,2}$

${ }^{1}$ Cumming School of Medicine

${ }^{2}$ Department of Clinical Neurosciences, University of Calgary, Calgary, AB

Background: Progressive ataxia and palatal tremor (PAPT) is a rare neurological disorder predominantly characterized by slowly progressive ataxia, dysarthria and palatal tremor. The number of cases of PAPT described in the literature is low and although the pathogenesis of this disorder remains unclear recent reports suggest that PAPT may be a tauopathy.

Case presentation: We present a case of a 76-year-old Mandarin-speaking man of Chinese descent who was referred to the Calgary Movement Disorders Clinic with a two year history of progressive gait ataxia as well as mild clumsiness, slurring of speech and dysphagia. His initial neurological examination revealed saccadic pursuit with normal horizontal and vertical saccades, cerebellar form of dysarthria, palatal tremor, mild appendicular ataxia and mild to moderate gait ataxia. There was no cognitive impairment, pyramidal or extrapyramidal involvement or autonomic dysfunction. Past medical history was significant for hypertension, hypothyroidism and atrial fibrillation. Family history was negative for similar presentations. His MR brain showed bilateral olivary hypertrophy with T2 hyperintensity. Based on his neurological examination, investigations as well as clinical course, a diagnosis of sporadic PAPT was made. As suspected, mild progression was noted clinically over the following three years of follow-up.

Discussion: Given recent case reports, PAPT likely represents a sporadic tauopathy. Other clinical entities may resemble PAPT but are typically distinguished by additional clinical features (including age of onset), distinct neuroimaging patterns or positive family history. 


\section{Traumatic Recreational Eye Injuries and the PROTECT Initiative}

Ammar Mahmood ${ }^{1}$

Imaan Kherani ${ }^{2}$

April Ingram²

Amin Kherani ${ }^{1}$

${ }^{1}$ Cumming School of Medicine, University of Calgary, Calgary, $\mathrm{AB}$

${ }^{2}$ Southern Alberta Eye Center, Calgary, AB.

Introduction: Traumatic recreational eye injuries represent a significant source of preventable injury. Sports such as baseball, squash and hockey, among others, can result in severe and irreversible ocular damage due to their high impact nature and involvement of projectile objects. Additionally, these ocular sports injuries present at a wide spectrum of ages, which unfortunately can result in lifelong functional impairment. Though a large number of these high-risk sports have protective eyewear available to participants, compliance and actual usage can be variable. As such, further efforts must be directed at preventative measures to raise awareness in the sporting community regarding the severity, irreversibility and frequency of sporting eye injuries. One such initiative is the Prevention of Recreational Ocular Trauma by Educating the Community Together (PROTECT). The objective of the PROTECT initiative is to target youth and raise awareness of the severity of recreational ocular injuries that can occur when individuals fail to wear proper eye protection. The purpose of this project is to outline a recreational sporting injury through a case study and discuss how it could have been potentially averted through preventative measures.

Case report: A 19-year-old healthy male with no previous ocular history sustained a traumatic eye injury while exercising at the gym. During his workout, the resistance bands he was utilizing broke unexpectedly, resulting in recoil of the band and a blunt force injury to his right eye. Visual acuity assessed at the Rockyview Urgent Eye Clinic was 20/200 in his right eye and 20/25 in the left eye (both with pinhole correction), with complaints of flashing lights, visual floaters and blurred vision. Slit lamp examination revealed injuries to the right eye including corneal abrasion, hyphema, traumatic mydriasis and perioribital swelling. A dilated fundus examination revealed a traumatic macular hole in the right eye, which was treated approximately seven weeks later with vitrectomy, intravitreal kenalong injection, membrane peel, intravitreal air exchange with $10 \% \mathrm{C} 3 \mathrm{~F} 8$ and posterior chamber intraocular lens implantation.
Thirteen days post-operatively, this patient had an improvement of the right macular hole and traumatic hyphema and iritis; however, vision in the right eye remained 20/200 (with pinhole correction). Though it is early to the assess visual acuity in the right eye post-operatively, there is a possibility this may represent his best visual acuity in the right eye.

Conclusion: This case highlights the tremendous importance of education and community outreach to prevent recreational ocular injuries. With adequate protective eyewear, this young patient could have potentially prevented severe retinal damage. Despite the immense skill of retinal surgeons, there are instances were irreversible ocular damage may occur with lifelong reduction in visual acuity. As such, initiates such as PROTECT, which was launched in May 2014, are targeted at providing awareness to the community on the importance of wearing ocular protection during recreational activity.

\section{Access to healthcare in the Himalayas: A case study on tribal areas of Himachal Pradesh, India}

Inderjeet S. Sahota, $M \mathrm{Mc}^{1,2}$

Bhavdeep S. Rehal BSc $\mathrm{c}^{1,3}$

Nidhi S. Panwar, MA ${ }^{1,4}$

1 Thousand Metres Above Foundation, Cumming School of Medicine, University of Calgary, Calgary, AB

2 Faculty of Nursing, University of Calgary, Department of Political Science, University ofCalgary

Introduction: Towns in the Indian Himalayas are some of the most geographically isolated in the world. Limited infrastructure development, coupled with low population density and geographic constraints, means access to health services is limited. However, the specific barriers that impede access to healthcare in this region have not been well studied.

Methods: We surveyed 83 adults who lived in eight high-altitude $(>2,000 \mathrm{~m})$ towns in the remote Himalayan districts of Shimla, Kinnaur and Lahaul and Spiti in eastern Himachal Pradesh, India. Questions related to income, education, occupation, financial burden of healthcare, distance to nearest health facility, physician visits and perceptions of public vs private care were asked in Hindi or English as per the participant's preference. A detailed medical history was also obtained.

Results: Mean age of our respondents was $35.6 \pm 1.3$ years and 69 of the 83 respondents were male. Mean monthly income was $8,832 \pm 7,203 \mathrm{Rs}(\$ 180 \mathrm{CAD})$. Half $(50.6 \%)$ of the 
respondents had completed high school equivalence or higher; $14.47 \%$ had never seen a physician and of those who had, the mean time since previous visit was 19 months; $58.1 \%$ of respondents must travel by foot to seek medical services and of those that traveled by foot, the average length of time to reach the closest health post was $31.29 \pm 46.34 \mathrm{~min}$; and $18.3 \%$ of respondents had current health issues, the most common of which were cardiovascular, respiratory, MSK and gastrointestinal disease. Mean monthly income towards healthcare was $786 \pm 1,443$ Rs with a range of 0 to 6,000 Rs/month.

Individuals who used private care had significantly greater health expenses $(2,316 \pm 2,018.5$ Rs vs $423 \pm 1,005$ Rs; $\mathrm{p}=0.004)$. There was no significant difference in overall monthly income between those who used public care vs. those who used private $(8,217 \pm 6,744$ Rs vs $10,578 \pm 8,493 \mathrm{Rs}$; $\mathrm{p}=0.283$, respectively). Qualitative analysis found that lower cost, quality of services, and distance to nearest facility were the three major factors in determining whether to choose public vs. private healthcare.

Conclusion: This study examined, for the first time, the socioeconomic and geographic factors related to access to health in the high altitude districts of Himachal Pradesh, India. These findings provide insight into social factors influencing health access in other high altitude and remote regions of the developing world.

\section{A case study: HSP within the geriatric population}

Luxey Sirisegaram

Emily Kwan MD FRCPC MScCH

Introdution: Henoch-Schnolein purpura (HSP), also known as Immunoglobin A vasculitis, typically involves IgA-mediated leukocytoclastic vasculitis that targets small vessels, and manifests itself as palpable purpura, arthralgia/arthritis, abdominal pain and/or renal disease. The incidence rate of HSP in the adult population is $0.8-1.8 / 100,000$, and was found to be $2-33$ times more common during pediatric years, especially in children between 3-12 years of age. Although HSP has been known to be self-limiting in the pediatric population, the course of the disease is relatively unknown within the adult sphere-specifically within the geriatric population.

Case study: This case study reviews the case of an 85-year-old Caucasian male who presented with a second manifestation of HSP within the last two years. His history included a $10+$ year history of anemia of unknown origin accompanied with a month long history of an erythematous macular rash bilaterally on the lower arms. Additionally, uriticarial wheals, ecchymoses and palpable purpura were seen in crops distributed bilaterally and symmetrically on his lower extremities, as well as buttocks and groin. HSP was confirmed using skin biopsy confirmed leukocytoclastic vasculitis with direct immunofluorescence studies stain of $\operatorname{Ig} \mathrm{A}$ deposition.

Conclusion: Although HSP is considered primarily within the pediatric sphere, it is a disease process that can manifest itself within the geriatric population, and must be considered in a situation in which a member of the geriatric population presents.

\section{Delayed postpartum hemorrhage due to uterine artery pseudoaneurysm following Caesarean section}

Hai Chuan $\mathrm{Yu}^{1}$

Nicole Hubner ${ }^{1,2}$

Kathryn M. Kenny ${ }^{1,2}$

1 Cumming School of Medicine, University of Calgary, Calgary, $\mathrm{AB}$

2 Department of Obstetrics \& Gynecology, Cumming School of Medicine, University of Calgary, Calgary, $\mathrm{AB}$

Introduction: Delayed postpartum hemorrhage (PPH) refers to excessive blood loss between 24 hours and six weeks postpartum. The overall incidence of delayed $\mathrm{PPH}$ is $0.5 \%-$ $1.3 \%$, and shares many of the common etiologies with early $\mathrm{PPH}$, including uterine atony, retained products, trauma, as well as coagulopathy. Delayed PPH can also arise from less common causes, such as arteriovenous malformations, surgical wound dehiscence, and choriocarcinoma. Here we report here a case of delayed PPH secondary to Caesarean section.

Case presentation: A previously healthy 35 -year-old gravida 2 , para 2 woman presented to the emergency department on post-operative day 9 following an uncomplicated Caesarean section. Approximately 12 hours prior, the patient experienced severe pain at her incision site. She subsequently passed large clots vaginally, followed by brisk bleeding in the emergency department. Her vital signs were initially stable, with a hemoglobin level of $133 \mathrm{~g} / \mathrm{L}$. Several hours later, she became hemodynamically unstable, and was found on exam to have lost approximately $1,000 \mathrm{~mL}$ of blood. She was taken to the operating room where vaginal and uterine pathology could not be visualized, and brisk bleeding persisted despite uterine massage and tamponade via balloon catheter. The patient was taken for embolization by interventional radiology, and was 
found to have a pseudoaneurysm arising from the left uterine artery. Successful embolization yielded hemostasis, and the patient recovered with no further complications.

Conclusions: While our patient received the appropriate treatment and recovered without adverse outcomes, early diagnosis of her source of bleeding would have allowed for early definitive treatment and helped avoid the need for urgent surgical management. Given the high rate of mortality associated with post-partum hemorrhage, it is crucial for physicians to not only consider and treat for the common etiologies, but also to maintain a wide differential diagnosis and entertain the possibility of a rare etiology as the cause of the patient's presenting symptoms. When early diagnosis is not possible, as in our case, having an algorithmic and progressive approach to management of delayed $\mathrm{PPH}$ is important to successfully manage the patient's bleeding and hemodynamic stability.

\section{Psychosis in mild cognitive impairment: A systematic review and meta-analysis}

\section{Zahinoor Ismail \\ Heba Elbayoumi \\ Marium Arain \\ Scott Patten \\ Eric Smith \\ Kirsten Fiest \\ Tom Schweizer \\ Colleen Millikin \\ Corinne Fischer \\ David Hogan}

Mathison Centre for Mental Health Research \& Education, Hotchkiss Brain Institute, University of Calgary, Calgary, AB

Introduction: The prevalence of dementia is on the rise and approximately 35.6 million people are currently suffering from dementia worldwide. Previous research has shown a high prevalence of psychosis, including hallucinations and delusions in Alzheimer disease (AD) patients (41\%), with a link to higher cognitive decline. The importance of early detection of psychosis in the $\mathrm{AD}$ population is highly emphasized, as these symptoms seem to increase during the first few years. It is equally important to understand the epidemiology and severity of these symptoms in aging populations to better understand the corresponding neurobiology as well as the natural history and to better guide therapy and outcomes.
Objective: Review literature to update about the best estimate of psychosis prevalence in mild cognitive impairment (MCI) and dementia populations.

Methods: A systematic search of literature up to February 2014 was performed using Medline, Embase and PsycINFO databases, with no restrictions on the year of the study. Inclusion criteria included original research studies, studies reporting on psychosis in MCI or dementia and studies in English language only. References were retrieved to a software reference manger. A team of 10 reviewers participated in all the phases of screening the abstracts, full text article review and the data abstraction. Every reference and selected full text article was double reviewed by two independent reviewers. The Full Text Inclusion criteria include: 1- The study reports on original research. The study's primary group of participants were MCI, dementia or both. The study reports the prevalence of psychosis (hallucinations, delusions, or both) in this population.

Data extraction and synthesis: Before beginning, an online data abstraction form was developed and pilot-tested in a random sample of studies, to ensure that all the study-related variables were captured. Data abstraction for every study was completed by two independent reviewers. A completed form for each article was submitted. All studies meeting inclusion criteria at this stage were then reviewed by final reviewer (ZI) to ensure appropriateness for inclusion in the final analysis.

Main outcome and measures: The primary outcome is to precisely estimate the prevalence of Psychosis (delusions and/or hallucinations) in MCI and in dementia.

Results: Currently, we are in the process of data abstraction and analysis and will be presenting the results in the poster presentation on Oct 30, 2015.

\section{Who is assisting assisted reproductive technology? Review of international funding policies}

Valerie Bloomfield ${ }^{1}$

M Dixon $^{2}$

${ }^{1}$ University of Calgary, Calgary, AB

2 University of Toronto, Toronto, ON

Introduction: Nearly 4.3 million children have been conceived through assisted reproductive technology (ART) worldwide over the past three decades. Transfer of multiple embryos has resulted in increased rates of twin and triplet births, at a significant cost to maternal and fetal health and to the healthcare system. As such, governments are implementing 
new initiatives and public funding policies to promote elective single embryo transfer. Through this study, we aim to compare international policies governing ART practice and funding models.

Methods: Preferred sources for ART data collection included national registries, health ministries and academic centers. When available, we captured the total number of ART cycles, cycles with single and multiple embryo transfer, singleton live birth rate and multiple birth rates per delivery. Health policy was capture from government sources and literature review.

Results: In total, data was collected from 18 high-income countries. According to most recent data, $28 \%$ of cycles were single embryo transfer, with a mean of 1.9 embryos transferred per cycle. Overall, $80 \%$ of live births resulting from ART were singleton deliveries. Fifteen nations legislate the number of embryos that can be transferred per cycle, with the remainder of nations providing guidelines through national regulatory bodies. Five nations (Belgium, France, Greece, Slovenia and Sweden) offer comprehensive funding for women undergoing fertility treatments, with 12 additional nations offering partial coverage to eligible women and couples.

Conclusion: The use of ART continues to grow in high-income countries. A shift towards public funding has been seen against the backdrop of several healthcare models. Countries with supportive, publicly funded programs have increased the rate of single embryo transfers. Further studies are necessary to follow health outcomes as public funding becomes more widely available to women struggling with infertility.

\section{The integration of new innovations into existing systems of surgical aid: Ensuring sustainability, affordability, accessibility, acceptability and applicability}

Rebecca Buttar, MSc

Institute for Global Health, University College London, London, England

Introduction: Surgically-treatable conditions are estimated to contribute to $11 \%$ of the global burden of disease and $15 \%$ of the global disability-adjusted life years. Unfortunately, $10 \%$ of the global population receives $90 \%$ of the available surgical services. Contrary to what funders may think, surgical care has been proven to be a cost-effective pillar of public health. As a result, the World Health Organization has called for surgical treatment of pregnancy, injury, emergency and disability prevention to be included in basic public health packages. Recently, surgical organizations are reflecting back on their mechanisms of care to come up with guidelines for making their care delivery systems safer and of better quality.

Methods: A literature search was conducted using a structural review method in conjunction with snowballing, where searched literature led to further sources. The structured literature review involved a thorough search of two databases, EMBASE and PubMed, which were selected for their relevance to medicine and biomedical data. A mixture of grey literature was used for background reading and to supplement the literature search. Thematic analysis was undertaken to group findings into broad categories of surgical aid implementation objectives.

Results: The structured literature search identified a total of 775 papers. When the inclusion and exclusion criteria were applied, 277 papers were found in EMBASE and 84 were found in PubMed. Thirteen of these were duplicates, which left 348 texts to be screened. Based on suitability screening, the amount of text was narrowed down to 59 , a further 16 texts were added from snowballing, leaving 75 papers to be analysed with additional sources used for supplemental and background reading. Thematic analysis generated groupings into broad categories of sustainability, accessibility, acceptability and applicability.

Conclusion: The practice of surgery overseas can be carried out in an ethical manner with careful planning and the respect of a few guidelines. Just as there is a push to allow life-saving pharmaceuticals to cross political and geographic borders, so too should there be advocacy to ensure that the lifesaving treatments that have been known and available for decades in the developed world are available to those in need living in the developing world.

\section{Impact of long-acting injectables versus oral antipsychotics on hospitalization rates in patients with schizophrenia: A retrospective review}

Bharat Chawla, MD

Michelle Bell, RN

Vanika Chawla

Background: In schizophrenia, lack of treatment compliance is a major issue leading to costly outcomes such as increases in relapse and hospitalizations. Long-acting injectable (LAI) formulations of antipsychotic medications were developed to 
improve non-adherence and reduce relapse; however, LAI use remains relatively low in Canada. Although LAI costs are initially higher than orals, studies have shown a reduction in negative outcomes associated with LAI use compared with orals, resulting in overall savings to health care system. In our study at the Peterborough Regional Health Centre (PRHC), we hypothesized that LAI use would be associated with decreased hospitalizations and length of hospital stay compared with oral usage.

Method: A retrospective chart review, using a mirror-image design, examined hospitalization rates in outpatients $(\mathrm{N}=22)$ one year before and one year after initiation of LAIs (risperidone-LAI and paliperidone palmitate) following oral treatment. Paired t-tests were used to examine potential differences between pre- and post-LAI initiation.

Results: Overall, a total of 94 vs seven hospital admissions on orals vs. LAIs, respectively, were observed. The mean $( \pm \mathrm{SE})$ number of hospital admissions (ER and Ward) per patient was four $( \pm 0.72)$ on orals and $0.3( \pm 0.22)$ on LAIs $(p<0.001)$. The mean $( \pm$ SE) length of stay per patient in hospital on oral therapy was 35 days $( \pm 5.37)$ vs one day $( \pm 0.92)$ for LAI treatment $(p<0.05)$. Cost benefit analysis, using data from the Ontario Drug Benefit and cost estimates of ward and ER admission at PRHC, estimated the difference before and after initiation of LAI use as a net savings of $\$ 474,272$ for these 22 patients.

Conclusions: Results from this study indicated that conversion from oral antipsychotics to LAIs was associated with significant reductions in hospitalizations and length of stay; an observation that was associated with substantial reductions in health care costs.

\section{Scoping review: Quality of life in older adults living with HIV in sub-Saharan Africa}

\section{Samantha Cheuk ${ }^{1}$}

Stephanie Nixon ${ }^{2,3}$

Patty Solomon ${ }^{4}$

1 Department of Global Health, McMaster University, Hamilton, $\mathrm{ON}$

2 International Centre for Disability and Rehabilitation (ICDR), Toronto, ON

${ }^{3}$ University of Toronto, Toronto, ON

${ }^{4}$ School of Rehabilitation Science, Master University, Hamilton, $\mathrm{ON}$
Introduction: With 24.7 million people infected with HIV, sub-Saharan Africa is the hardest hit region in the world accounting for just under a third of the world's HIV-infected population. With the availability of antiretroviral therapy (ART), people who are HIV-infected and can tolerate treatment are now leading longer lives. In 2014, UNAIDS published the Gap Report that identified adults aged 50 years and older as a vulnerable group being left behind in HIV treatment and prevention efforts. To address the gap in knowledge in the HIV, aging and disability literature, a scoping review was conducted to determine what is currently known about the quality of life of older adults living with HIV in sub-Saharan Africa (SSA).

Methods: The purpose of the scoping review was to map out published literature on quality of life among older adults living with HIV in SSA. Following Arksey and O'Malley's framework for conducting scoping reviews, six databases were searched for papers that captured an overlap of four concept domains: HIV/AIDS, older adults, sub-Saharan Africa and quality of life. Synonyms were used for each concept domain and databases were searched systematically. Only articles that met specified criteria of quality of life of older adults living with HIV in sub-Saharan Africa were included in the study.

Results: The database search yielded a total of 1,021 titles and abstracts that were screened using the inclusion and exclusion criteria. A final total of eight articles included for review and synthesis. Four studies utilized quantitative approaches while the other four used qualitative approaches to study different facets of quality of life among older adults living with HIV. Of the studies identified, quantitative studies pointed to minute differences in health and functional status between HIV positive and HIV negative older adults in sub-Saharan Africa while qualitative studies focused on contextual factors such as poverty that illuminated a greater burden to the quality of life of HIV positive older adults.

Conclusion: The review confirmed a dearth of research literature on older adults living with HIV in SSA as included studies were only conducted in two out of 47 countries in the region. The findings from this scoping review advance dialogue about the quality of life and experiences of disability in older adults living with HIV in SSA and points to a need for further research so that fewer people are left behind. 


\section{A phylogenic review of pain sensitization: Adaptive or maladaptive?}

Eun-Young Kang ${ }^{1}$

John Jarrell ${ }^{2}$

1 Cumming School of Medicine, University of Calgary, Calgary, $\mathrm{AB}$

2 Department of Obstetrics and Gynaecology, University of Calgary, Calgary, $\mathrm{AB}$

Introduction: Chronic pelvic pain affects over $25 \%$ of gynecologic patients and has been associated with pain sensitization; a process whereby non-responsive neurons become responsive or neurons either produce responses of higher magnitude or respond at a lower threshold. While sensitized women have been shown to display behaviours that seem maladaptive in the context of evolution, such as difficulties with sexual function or depression and anxiety, recent studies suggest that pain sensitization may confer an evolutionary survival benefit. Thus, the aim of this paper was to review the available evidence on pain sensitization studied in model organisms and to determine whether behaviours that sensitized animals demonstrated were adaptive or maladaptive. Methods: Medline and PubMed databases were used to identify appropriate articles. The species used for the study, method employed to yield and measure pain sensitization, and whether the observed findings suggest that pain sensitization is an adaptive or a maladaptive process were recorded in a table.

Results: Most studies showed likely adaptive behaviours, and some studies showed potentially maladaptive behaviours, whereas other studies found both adaptive and maladaptive behaviours in the same organism.

Conclusion: Upon comparing these behaviours with that of women with chronic pelvic pain, and based on the current evidence showing the plasticity of central pain sensitization in women, this suggests that pain sensitization in both animals in women may be a plastic phenomenon that is continuous, rather than dichotomous. These findings will aid in better comprehending the potential role of pain sensitization in women in the context of reproduction and evolution, and ultimately allow for further understanding of the mechanisms underlying chronic pain, which has great potential to impact maternal health and subsequently children's health.
Case report on the use of 5-azacytidine in the treatment of blastic plasmacytoid dendritic cell neoplasm and a review of the literature

Raida Khwaja

Carolyn Owen

Cumming School of Medicine, University of Calgary, Calgary, $\mathrm{AB}$

Introduction: Blastic Plasmacytoid Dendritic Cell Neoplasm (BPDCN) is a rare type of acute leukemia characterized by solitary or multiple skin lesions of macules and nodules with systemic manifestations. Currently, there is no standard treatment approach for BPDCN, despite its very poor prognosis. The treatments that have been used and reported in the literature include Acute Myeloid Leukemia (AML) / Acute Lymphoblastic Leukemia (ALL)-type treatment protocols. Allogeneic or autologous hematopoietic stem cell transplantation (HSCT) has been performed following induction therapy mostly in children and appears to be the only curative therapy for BPDCN; however, HSCT is only feasible for young, fit patients due to the nature of aggressiveness of the treatment while BPDCN primarily affects the elderly. Five-azacytidine (azacytidine) is a hypomethylating agent approved for the treatment of intermediate and high-risk myelodysplastic syndrome (MDS) and AML. As many patients with BPDCN are reported to die from AML and the disease is classified as a myeloid neoplasm, we hypothesized that azacytidine might be an effective and tolerable therapy for older BPDCN patients, unfit for curative chemotherapy and HSCT. Here we describe three cases in which patients were treated with first-line azacytidine for BPDCN. We did a review of the current literature to report what treatment is used for the management of BPDCN in the elderly.

Methods: A literature search was performed using PUBMED to obtain a general understanding of the current treatment strategies used clinically for BPDCN in the elderly and 23 relevant cases were found. Following that, we analyzed the efficacy of using azacytidine for BPDCN, retrospectively, in our three patients in achieving remission and preventing relapse. We assessed the benefit and risk of using azacytidine for BPDCN based on literature search and also our case reports.

Results: Despite loss of disease control after six or seven cycles of azacytidine in two of our patients, both survived $>12$ months, similar to survivals reported in other BPDCN cases. 
One of the three patients is still on remission ( $>19$ months). Thus, azacytidine leads to similar survival compared with what is reported and is a reasonable choice in the management of patients with BPDCN deemed unfit for intensive chemotherapy.

Conclusion: These case reports and review of the literature suggest that azacytidine is insufficient as monotherapy to prolong survival in BPDCN, except possibly in cases with very limited disease. The average overall survival of our patients (and the three other reported patients) remains around a year, similar to other reported case series. This therapy was less intensive and more tolerable than many suggested treatment options and remains a reasonable option for the treatment of elderly patients with BPDCN who are unfit for more intensive therapies. Additionally, we can hypothesize that a better approach to treatment might include a strategy of disease debulking with moderate intensity therapy (ex. CHOP) followed by azacytidine as maintenance therapy.

\section{Using geospatial modelling to evaluate access to emergency stroke care in Ontario}

Lauren Jewett ${ }^{1}$

Ario Mirian ${ }^{2}$

Demetrios J. Sahlas ${ }^{3}$

${ }^{1}$ Department of Medicine, McMaster University, Hamilton, $\mathrm{ON}$

2 Cumming School of Medicine, University of Calgary, Calgary, $\mathrm{AB}$

${ }^{3}$ Division of Neurology, Department of Medicine, McMaster University, Hamilton, ON

Background: The administration of thrombolytic therapy, such as intravenous tissue plasminogen activator ( $\mathrm{tPA}$ ), to eligible patients with an acute ischemic stroke can rescue brain tissue and improve outcomes. The emergency treatment of acute ischemic stroke in Ontario is currently provided through a network of stroke thrombolysis centres, some of which utilize telemedicine consultations (Telestroke).

Purpose: Our objective was to evaluate the access that the public has to stroke thrombolysis.

Methods: A literature review was conducted by searching Medline and EMBASE databases to identify literature that utilizes geographic analysis to assess general population access to thrombolytic therapy for acute stroke patients. Population data by dissemination area was used to overlay polygons created by Service Area Analysis using ArcGIS 10.1. The service areas are based on the Ontario Roads Network and Ontario Cartographic Boundaries. Geographic regions were divided into predefined driving times towards stroke centres due to the time-sensitive nature of stroke thrombolysis. Centres were categorized as being able to administer stroke thrombolysis independently, or utilizing the assistance of the Telestroke program. Catchment areas with and without the impact of the Telestroke program were compared.

Results: Based on the conducted literature review, no article has investigated the impact of Telestroke networks on geographic access to stroke thrombolysis for a patient population. Of the $12,857,821$ people living in Ontario in 2011, 99.83\% have timely access to stroke thrombolysis, leaving 21,829 people $(0.17 \%)$, in Northern Ontario and James Bay coastal communities, without access. The majority $(71.86 \%)$ of the population is within a 30 -minute drive of a Regional or District centre, increasing to $91.28 \%$ when the Telestroke program is included, for an additional 2,501,121 people; whereas $1.85 \%$ of the population have access only through the extended time window (between 3 to 4.5 hours), increasing to $3.86 \%$ with Telestroke, for an additional 258,618 people.

Conclusion: The vast majority of people in Ontario have access to stroke thrombolysis. The Telestroke program improves timeliness of access for those in Southern Ontario, yet rural and Northern communities remain without access to stroke thrombolytic care. Our analyses may provide insight for the Ontario Telestroke Network's decisions on future expansions in coverage by identifying the aforementioned underserved populations.

\section{A systematic review of applanation tonometer disinfection methods}

Alex Ragan ${ }^{1}$

Stephanie Cote ${ }^{1}$

John Huang ${ }^{2}$

1 Cumming School of Medicine, University of Calgary, Calgary, $\mathrm{AB}$

2 Division of Ophthalmology, Department of Surgery, Cumming School of Medicine, University of Calgary, Calgary, $\mathrm{AB}$

Introduction: The Goldmann Applanation Tonometer (GAT) is the gold standard instrument for the measurement of intraocular pressure. The instrument is inexpensive and 
convenient to use. Accordingly, it is found almost ubiquitously within the setting of ophthalmological practice with approximately 122 million patients undergoing tonometry with the GAT annually; however, tonometry performed using the GAT has been implicated in outbreaks of epidemic keratoconjunctivitis and it has been postulated that the instrument could similarly act as a vector for other infectious diseases. Accordingly, health regulatory authorities throughout the world have been reassessing their guidelines on the disinfection of the GAT apparatus. While the current standard of practice in Canada has been the use of $70 \%$ isopropyl alcohol swabs to clean the applanating tip of the tonometer head, far more stringent guidelines are being promulgated with the aim of enhancing clinical disinfection practices. In Alberta, these guidelines include the use of $2 \%$ accelerated hydrogen peroxide for five minutes or $0.525 \%$ sodium hypochlorite solution. Our research seeks to assess the extent to which the scientific literature supports the techniques recommended in the new disinfection guidelines.

Methods: A systematic review of the following databases was conducted for primary level scientific literature testing the effectiveness of different disinfection techniques and agents for the disinfection of GAT prisms: PubMed, Medline, Google Scholar, Web of Science, EMBASE, CINAHL, BIOSIS Previews and hand searches of reference tables. No restrictions were placed on search parameters regarding language, date, type of study (whether in vivo or in vitro) or the publication status of the study.

Results: Our review revealed 12 primary level studies that related specifically to the disinfection of GAT prisms. These studies were all performed in in vitro conditions. These studies confirm that disinfection with hydrogen peroxide and sodium hypochlorite are effective in disinfecting GAT prisms from pathogens that are common or particularly dangerous; however, the studies also suggest that disinfection with $70 \%$ isopropyl alcohol will achieve the same ends.

Conclusion: The proper disinfection of medical instruments is of paramount importance and the techniques and materials used to achieve disinfection should be supported by appropriate scientific evidence and with an eye toward the efficient use of scare resources. Our review of the scientific literature found that there is evidence to support the use of hydrogen peroxide and sodium hypochlorite for the disinfection of GAT prisms; however, the literature also supports the use of $70 \%$ isopropyl alcohol wipes for this purpose. These wipes are more economical and a similar effectiveness of disinfection. Accordingly, we do not support the revised guidelines as they encourage the use of a less economical process for similar results.

\section{Adverse drug events associated with administration of common asthma medications in children}

Arissa Sperou ${ }^{1,2}$

James Leung 3

Lisa Hartling ${ }^{1,2}$

Antonia Stang ${ }^{1,2}$

David Johnson

1 Cumming School of Medicine, University of Calgary, Calgary, $\mathrm{AB}$

2 Division of Emergency Medicine, The Alberta Children's Hospital, Calgary, AB

3 Department of Pediatrics, The University of Alberta, Edmonton, $\mathrm{AB}$

Introduction: Iatrogenic harm from medication administration is prevalent, potentially serious and often preventable. An adverse drug event (ADE) occurs when harm befalls a patient because of a drug exposure. ADEs encompass both adverse drug reactions (ADRs) and medication errors. Vulnerable patient populations, such as children, are at high risk of experiencing ADEs; however, there is little information regarding the incidence of ADEs in children, even amongst commonly administered medications such as asthma medications. Wheeze is a common presentation to pediatric emergency departments, and empiric therapy with asthma medication is often given despite the underlying etiology. The primary objective of this systematic review is to elucidate the incidence of ADEs associated with common asthma medications prescribed to children to achieve or maintain control of wheeze.

Methods: A systematic review of the literature was conducted to elucidate the incidence of ADEs in children administered asthma medications. The first step involved conducting a systematic search of the literature to identify articles. We searched six databases including Medline, Pubmed, EMBASE, International Pharmaceutical Abstracts, Web of Knowledge and CENTRAL. The second step involved applying eligibility criteria to articles identified in the search. In this process, two independent reviewers read the identified articles and excluded ones that did not meet inclusion criteria. The third step involved conducting data extraction and verification of the included studies by two independent reviewers. 
Results: The initial search yielded 8,026 potential articles. After review of the identified articles' titles and abstracts, 7,328 articles were excluded thereby leaving 698 articles for full text review. After reviewing the full text of the 698 articles, it was determined that only 38 articles met inclusion criteria. ADE incidence data were extracted and verified by two independent reviewers. Additionally, the quality of the 38 studies was assessed and verified by two independent reviewers. Analysis of the extracted data is currently ongoing; however, preliminary review of the extracted data suggests that often only adverse drug reactions are reported in the literature and there is absent or poor documentation of medication error. Additionally, standardized methods to assess causality, severity and preventability are often either not used or not reported on. Conclusions: Analysis of the extracted data is currently ongoing. We expect analysis of the data to be completed within the next couple of months. 